\title{
THE PATHOGENESIS OF ACUTE RENAL FAILURE ASSOCIATED \\ WITH TRAUMATIC AND TOXIC INJURY. RENAL ISCHEMIA, NEPHROTOXIC DAMAGE AND THE ISCHEMURIC EPISODE ${ }^{1}$
}

\author{
By JEAN OLIVER, MURIEL MAcDOWELL, AND ANN TRACY \\ (From the Department of Pathology, The College of Medicine, State University at \\ New York City, Brooklyn, N. Y.)
}

(Submitted for publication July 16, 1951 ; accepted July 30, 1951)

\section{THE NATURE AND SCOPE OF THE PROBLEM}

Shortly after the publication in 1941 of his study of the "crush kidney," the late John Shaw Dunn sent tissue showing the peculiar tubular lesion that characterizes this condition to the senior author for microdissection. Somewhat later similar material was received from Dr. J. Edgar Morison of Belfast and this was followed by the gift of a considerable collection of examples of that larger group of renal lesions which was later to become known as "lower nephron nephrosis" from Dr. Baldwin Lucké.

Since that time a continuing study of these perplexing forms of renal damage has been in progress in this laboratory as confreres too numerous to thank here in person have sent kidneys both from their clinical cases and experimental investigations. Among the latter was a series of dog kidneys from Drs. Donald Van Slyke, R. A. Phillips and their co-workers in which the functional lesion of the "kidney of shock" had been reproduced and studied in great detail by clearance methods.

The result of these ten years of work in an everwidening area of investigation had been chiefly the accumulation of a bewildering cloud of apparently unrelated minutiae in which little evidence of a pattern of unity of pathogenesis or functional correlation could be discerned. As is not infrequent in such situations, through no virtue of the present investigators other than that they persisted in their task of accumulating curiously damaged nephrons while awaiting illumination, the cloud was quite suddenly dissipated by the appearance of two related studies in renal pathology.

1 This work was supported by grants-in-aid from the Life Insurance Medical Research Fund, The American Heart Association, and the New York Heart Association.
The first of these came to us through the privilege of a study of Dr. Homer Smith's manuscript of Chapter XXIV (Acute Renal Failure Related to Toxic and Traumatic Injuries) from his monograph, The Kidney (1). This extended and profound analysis of the functional disturbances in the group we had been studying from the structural viewpoint allowed us by a sort of inductive reversal of the causal line to proceed back from functional "effects" to a confirmation of what we had hitherto dimly glimpsed as hypothetical structural "cause," namely a disturbance in renal blood flow. The successful conclusion of the present work may therefore be looked upon as derivative of his effort.

The suspicions of the morphologists in regard to the part that renal ischemia plays in the pathogenesis of the renal lesion, even when reinforced by the considerable support of the functionalist, might well have remained, as conclusions drawn from the static data of structural appearances so often do, in the realm of reasonable hypothesis, except for the discovery by Schlegel (2) of a method for visualizing the pattern of the renal circulation by the intravenous injection of a fluorescent dye into the living animal. As will be seen in our later consideration of the problem, this procedure made possible certain crucial experiments in which the pattern of the renal circulation could be correlated with various sorts of tubular damage and so confirmed the essential unity of our pathogenetic theory.

Our investigation began therefore with an examination of the structural status of the "crush kidney" and examples of "lower nephron nephrosis" and extended to cover all "Acute Renal Failure Related to Toxic and Traumatic Injuries." Our exposition will follow a similar course, not only from a desire to show the chronological pat- 
tern of our investigation but because, as will become apparent, the "crush kidney" furnished the key to the solution of the pathogenetic puzzle of the broader group.

No detailed review of the lengthy literature which concerns that form of renal damage which not infrequently follows the syndrome of shock need be restated, for the summaries, from the pathological side by Lucké (3) and Mallory (4); on its functional and experimental aspects by Van Slyke (5) and by Corcoran and Page (6); and more recently, on its clinical features by Burch and Ray (7), leave little to add. It is clear from these resumés that a most important syndrome characterized by grave renal damage and failure has been established, yet in a detailed study of them, difficulties of interpretation and correlation become so apparent as to leave the subject in the minds of most who are practically concerned with it, either as pathologist or clinician, in considerable confusion.

To set the problem clearly for our consideration it might be useful to summarize some of the difficulties which concern the pathogenesis of the renal lesions of this group and the correlation of its clinical and functional aspects with current descriptions of the structural changes that occur in the kidney.

As important in the pathogenesis of the tubular lesions the following factors are usually mentioned; (1) the presence of blood or muscle pigments in the tubules, (2) the hypothetical action of unknown "toxic products" derived from crushed tissues, and (3) the effects of local renal ischemia which is a reaction to the general circulatory disturbance of shock. To account for the functional disturbances, oliguria, anuria, and uremia, attention is usually called to (1) various sorts of structural, or assumed "functional," damage in the tubule walls, that might be reasonably supposed to allow back diffusion, (2) the presence of casts which might interfere with the flow of fluid through the tubules, and (3) the renal ischemia which might directly be the cause of decreased filtration.

To correlate with and to serve as an explanation of these pathogenetic and functional problems stands the current description of the pathological lesion in the kidney. This, as detailed by Lucké (3), is based on the original description by
Dunn, Gillespie, and Niven (8) of a peculiar lesion in the distal tubule. Lucké holds that this lesion is "unique and regularly attended by characteristic functional disturbances" and that the "location of the lesions is so characteristic and unique that the term 'lower nephron nephrosis' seems more descriptive ... (than) 'haemoglobinuric nephrosis" " ([3], p. 371). There are "relatively slight or no structural changes in the upper parts of the nephrons . . . renal corpuscles, proximal and intermediate segments. It is clear that the damage in this disease" (our italics) "is essentially confined to a particular component of the nephron. The identification of the several components is not difficult ;" . . . "Proximal segment. The appearance of the lining cells of the proximal segment is often quite normal" ... they may "exhibit cloudy swelling. Occasional fatty, deposits are disclosed... frank degeneration or actual necrosis is very rare. Intermediate segment. Definite changes can seldom be found in the intermediate segment; there is neither obvious cellular injury nor precipitation of haem compounds. Lower segment. It is widely agreed the lower segment is damaged selectively" (p. 386) and a description of the tubular lesions of Dunn, Gillespie and Niven follows. ${ }^{2}$

If one returns from this description of the structural status of the damaged kidney to questions of pathogenesis and functional correlation difficulties become apparent. The first of these is: what, even in the realm of hypothesis, could be considered a likely cause of such a unique and selective damage to a limited portion of a nephron? Certainly not renal ischemia which from our knowledge of the inter-tubular circulation would of necessity involve any or all parts of nephrons indiscriminately. Renal toxic agents act on the tubule epithelium because of its functional characteristics; mercury for example is absorbed, or on the remote chance secreted, by the cells of the proximal convolution and so they suf-

2 One finds in the literature some variation in opinion as to the localization of tubular damage; some investigators mention changes in the proximal convolution, others deny them. It would seem that these discrepancies arise from the use of a method of observation, the histological section, which is inadequate for the determination of the desired information, and since a method is at hand which can objectively settle the matter, they therefore need not be critically discussed. 
fer. There is no evidence with which we are familiar that suggests the existence of analogous functional mechanisms which could produce a specifically localized damage in ascending limbs and distal convolutions.

If heme pigments are responsible for the extreme damage to tubule walls, why are these substances found so innocuous in experimental procedures? Moreover, it is an established fact that these pigments are specifically absorbed by the proximal convolution (9) and yet this segment of the tubule is described as uninjured.

Can the damage in the distal convolution be due to the mechanical effects of the blockage by pigment casts or crystals? This is a factor that at least shows the desired specificity of location in the "lower nephron," for it is here that deposits and casts occur, but their presence in many other renal conditions in which no such localization of tubule damage is found makes it difficult to believe that they can be a significant factor in the production of the "specific" tubular disruption found in the kidney of shock.

Correlation of the clinical and functional disturbances of the syndrome with the uniquely characteristic lesion described for "lower nephron nephrosis" suffers similar difficulties of comprehension. Presumably tubule fluid might diffuse or leak from any part of the renal tubular system from glomerulus to the ducts of Bellini, but the proximal tubule has been shown to be responsible for 80 per cent of water absorption (10) and one wonders if it may not be involved in a syndrome which is characterized by oliguria or anuria. It might also be expected that an uniquely localized lesion would produce a recognizably characteristic functional disturbance. It is of interest in this regard that such a functional syndrome has been described (11) and even designated as "pure" lower nephron nephrosis, but this renal disturbance bears no relation to what occurs in the kidney of shock. The aetiological factor, renal ischemia, for which strong clinical evidence exists, though it might explain at least the early oliguria could not, as we have said, on any known anatomical basis account for a lesion limited to the "lower nephron." In fact it is regularly reported that experimental renal ischemia damages particularly the proximal convolution.
All the critical reviews of these matters emphasize these confusing complications and contradictions and end with an expression of the hope that more investigation, particularly along experimental lines, may lead to clarification. The assumption is apparently made, therefore, that the problem hás been correctly stated in its structural and functional aspects and that only elaboration of detail is needed for elucidation. But before more time is spent in the search for hypothetical toxic materials acting on a functionally obscure portion of the nephron by mechanisms as yet unknown it might be well to examine again more closely the relevancy of the fundamental question, for in the manner of its asking may well lie the source of our difficulties: can the status of the abnormal kidney in this syndrome be properly considered a renal disease that is characterized by an unique and specifically localized lesion in the nephron, which may be accurately described as "lower nephron nephrosis"?

In such situations a change in point of view is often helpful and that is the purpose of the present report. It details the results of the application of methods, designed to allow an inspection of the damaged nephrons in their entirety and continuity, to the study of the structural lesions in the kidney. It is obvious that such observations will be especially pertinent in a problem where specific localization of lesions is suspected.

\section{METHODS}

Though the mounting and staining of dissected nephrons has been a routine technique in this laboratory for many years, no publication, except for a general description of its use (12), has given the detail of the procedure.

Small blocks, 0.5 to $1.0 \mathrm{~cm}$. in thickness, of formalinfixed kidney are macerated in concentrated $\mathrm{HCl}$ at room temperature until they are quite soft. Depending on the amount of fibrous tissue in the specimen this will require from 12 to 24 hours. The macerated tissue is repeatedly washed by decanting in tap water to remove the acid and the nephrons are dissected while floating in water in a Stender dish under a binocular microscope. Individual nephrons are disentangled with needles, drawn up and arranged on a slide in the same manner as is employed with frozen sections and the excess water removed from around the specimen by blotting. A streak of vaseline is placed near each edge of the slide and a cover glass lowered upon it to form a cell which is open at each end; staining reagents may then be introduced by capillarity.

We have found the most generally useful stain to be 
iron-haemotoxylin. Ten per cent $\mathrm{Fe}_{2} \mathrm{Cl}_{3}$ is drawn through the cell with filter paper and allowed to stand for three to five minutes. It is then replaced by a 1 per cent aqueous solution of haemotoxylin for five to ten minutes. The densely black nephron is then differentiated under the microscope with a weak solution of $\mathrm{Fe}_{2} \mathrm{Cl}_{3}$. The degree of decoloration may be varied to suit the purpose of the staining and final repeated flushing with tap water completes the procedure. Microscopic examination of the uncleared nephron is made in water as we have found that any attempt to clear or mount the specimens in the usual media shrinks and distorts them. In a similar manner other stains may be used: fat is well shown by Quenzel's cadmium-Sudan III mixture and aqueous toluidine blue is useful for metachromatic materials. Histochemical methods such as the common reactions for $\mathrm{Fe}$ may also be done on the intact nephron.

As the specimens are mounted in water the stain is not stable but fades in a few days. A special method of microphotography has therefore been devised to record permanently our observations. As a magnification of from 100 to 200 times is necessary to show cellular detail and the tubule or nephron to be photographed may be from 1.0 to $1.5 \mathrm{~cm}$. in length, serial overlapping exposures on $31 / 4 \times 41 / 4$ Wratten $M$ plates are made as the specimen is moved across the microscopic field. Due to irregularities in focus and lack of optical flatness of field only the central third of each print is used in preparing the montage which shows the continuous structure. The overlap is therefore sufficient to allow adjoining prints to be cut on the bias and the direction and course of the tubule in its final mounted form can thus be directed to fit the form of a conventional illustrative plate with no loss of continuity. The original plates measure $18 \times 23$ inches but appear as reproduced for publication in a reduced magnification of 40 to $80 \times$. Considerable sacrifice of the finer detail has occurred in this economically necessary procedure.

A nephron well stained with iron haemotoxylin from tissue immediately fixed in 10 per cent formalin shows a remarkable amount of cellular detail in spite of the preliminary treatment with concentrated $\mathrm{HC} 1$ and the fact that it must be examined in a relatively thick ( 40 to $60 \mu$ ) uncleared tubule. The appearance of the various portions of the normal nephron are shown in Plates 1 and 2 . In summary, the nuclei appear as unstained vesicles due apparently to the extraction of their nucleic acid. It is interesting that the hyperchromatic nuclei of regenerating cells often stain heavily and show considerable detail of their chromatic structures, both chromosomes and nucleoli (Plates 16 and 25). Whether this contrast in the effect of extraction by $\mathrm{HCl}$ is due to quantitative or qualitative differences might be worthy of investigation.

The most characteristic cytoplasmic elements of the renal cell, the mitochondrial rodlets, persist though their form is changed from the long, filamentous structures that are seen in well-fixed histological sections to short, elongated granules which still maintain their original linear orientation. As it is necessary to examine them through the entire thickness of the tubule, their detail is somewhat confused but since mitochondrial elements are usually considered to be especially susceptible to the action of acetic acid, it is surprising to see how well they are preserved after the treatment with concentrated $\mathrm{HC} 1$ (Plates 2 and 28). Other cytoplasmic structures, such as the brush border are clearly shown (Plate 2C) and the basement membrane of the tubule stands out as a sharply defined, homogeneous, thin band that outlines the lateral aspects of the tubule (Plate 2A).

The examination of nephrons from routine human autopsies suffers the same but no more difficulties in cytological examination and interpretation than are encountered in preparations of this material by the technique of the histological section. With both methods, the mitochondrial pattern suffers from the unavoidable delay in fixation of the tissues. It has been found by experiment that 15 minutes at room temperature is sufficient to alter considerably the cytological detail, so that the most common appearance noted in the renal epithelium of normal dissected nephrons of human origin is that of a darkly stained cytoplasm filled with irregularly distributed fine "granules," and, as in histological sections, the brush border may be obscure. As will appear in our descriptions of the damaged nephrons, various cellular abnormalities, cloudy swelling, hyaline droplets, fat accumulation, vacuolar hydropic degeneration, pigment deposit, regeneration with the production of an atypical epithelium, cast formation, and all the other phenomena of renal tubule alteration and damage are seen in an analogous but very different aspect from what is apparent in the conventional pattern of the histological section. It is perhaps unnecessary to add that the latter method was used in all cases to supplement our study of the dissected nephrons.

\section{MATERIAL}

The material of this study is of three sorts; first, human kidneys from a large variety of examples of renal damage associated with the syndrome of "shock" and other forms of acute renal failure (Table I) ; secondly,

\section{TABLE I}

Clinical aetiology of 54 fatal cases of Acute Renal Failure

Crushing injury (bombing)

Burns

Transfusion accidents

Malarial blackwater fever

Obstetrical complications

(Eclampsia, hemmorhage, placental separation)

Surgical shock

(Perforated gastric ulcer)

Cold paroxysmal hemoglobinuria

Poisoning

Sulfonamides

Mercuric chloride

Di-ethylene glycol

Carbon tetrachloride

Potassium chlorate

Mushroom poisoning 
dog kidneys from a series of experiments in which the pathogenetic factors and functional disturbances following induced shock had been examined; and thirdly, rabbit kidneys from experiments designed to show the aetiological relations of the various factors involved in renal damage due to toxic injury. We shall present first the essential lesions that were found in the human kidneys and then endeavor to show how these lesions may be interpreted in the light of what was found under analogous experimental conditions.

THE STRUCTURAL CHARACTERISTICS OF THE LESION IN THE HUMAN KIDNEY IN ACUTE RENAL FAILURE ASSOCIATED WITH TRAUMATIC AND TOXIC INJURIES

Since the general nature of the clinical and pathological syndrome has been thoroughly examined and described by many investigators and these studies well summarized by Lucké (3) and others we have omitted much of our detailed case record description, both clinical and pathological, except where it was pertinent to some particular point in our exposition. This seemed permissible for little new has been added by our investigation to previous descriptions of the generality of the pathological status of the abnormal kidneys; a considerable addition results, however, from the different aspect of the lesions and from the integration of them into the general picture of renal damage that is made possible by their observation in the continuity of the complete nephrons.

There are two questions regarding the structural lesion with which we shall be especially concerned and both will be of crucial importance in the final interpretation of its pathogenetic and functional significance; first, the nature of the tubular lesions, and secondly, the distribution of these lesions not only in the individual nephron but among the nephrons generally. Subsidiary to these two problems are such matters as the nature and degree of tubular occlusion, the occurrence of interstitial tissue reaction, the presence and effect of deposits of pigment, and the reparative changes that may occur in the damaged nephrons.

\section{The essential tubular lesion-tubular disruption or tubulorhexis}

As in all examples of renal disease, a considerable variety of "tubular lesions" is present in the kidney when acute renal failure occurs as a result of traumatic or toxic injury. On this background of non-specific renal damage is found, however, a lesion peculiar to and therefore characteristic of the group, namely a localized destruction of the entire tubule wall. This lesion was first described in histological sections by Dunn and his co-workers in the crush kidney and its occurrence in other related conditions has been noted with emphasis in varying degree by all subsequent investigators.

The lesion is clearly shown in all the varied manifestations of its development in preparations of complete nephrons; as one follows the course of the intact tubule, quite suddenly a place is found where the basement membrane is broken, frayed or disintegrated and the epithelial lining disrupted and necrotic. The result is a solution of continuity which may include only a part of the wall of the tubule or which completely interrupts its course so that the fragmented remnants lie between a still intact and well preserved proximal portion and its distal prolongation (Plates 327). The lumen thus lies open to the intertubular interstitial tissue and its capillaries and veins (Plates 5 and 10).

The lumen of tubules so affected may or may not contain solid material in the form of debris or of a consolidated, well-formed cast. The frequency of association of rupture and cast is apparently determined by the situation of the lesion: if the former occurs in the ascending limb of Henle's loop or in the distal convolution where casts are frequent, the two are not uncommonly found together (Plate 5). That this association can, however, be little else than fortuitous is emphasized by the fact that large and extensive casts, many containing blood pigments, are frequently found in the distal convolution with no lesion in the tubule wall (Plates 7, 15, 17 and 19).

A great variation in the appearance of the disrupted tubule results not only from the extent of the damage and the presence or absence of solid material in its lumen but also from the reaction that may occur in both the tubule wall itself and in the mesenchymal tissue in the neighborhood of the rupture.

In certain instances a simple solution of continuity describes the lesion, as the tubule wall appears dispersed into fragmented debris and necrotic desquamated epithelial cells that are loosely held together by the thin, disintegrating basement membrane which still maintains in some degree 
the original contours of the tubule (Plates 6,8,14 and 23). There is little or no reaction in the peritubular tissue. In other examples, the interstitial reaction about the ruptured segment is extreme so that in the dissected specimen tufted masses of connective tissue fibers surround the damaged tubule and are so firmly attached to it that they cannot be removed with the dissecting needles (Plates 4, 9, 11 and 26). Or the surrounding reaction is seen as clefinite "granulation tissue" with a proliferation of capillaries among the mesenchymal elements that not only surround the ruptured tubule but grow into its disrupted lumen (Plates 5, 10 and 25).

The reaction of the epithelial cells to damage of the tubule wall will be considered in detail in a later description of the reparative processes that occur in the nephrons. As a rule the epithelial cells are remarkably well preserved up to the very edge of the tubular disruption and by their larger size and irregular shape show definite evidence of proliferation. At times an actual extension by growth of a thin, flattened epithelial margin may be observed accompanied by irregular clusters of atypical, hyperchromatic nuclei (Plates 16, 17, 19, 21, 24 and 25). However, as a result of the peculiar character of the lesion. the basement membrane being no longer present to support the proliferating epithelium, restitution of the damaged tubule wall seldonı results.

An examination of the illustrations of this unusual tubular lesion will show better than many words the variations that are built up of combinations of the elements we have described. No typical combination has been found in any particular clinical variety of the syndrome, though in any one case they may tend to resemble each other.

\section{The localization of the lesion of tubulorhexis}

Except for its three-dimensional aspect, our description so far of this characteristic tubular lesion does not differ essentially from that of previous studies based on its appearance in histological sections. When the second of its supposed characteristics is considered, i.e., its specific localization in the "lower nephron," a departure from current concept becomes apparent as the direct result of its examination in the continuity of complete nephrons.
In all of the kidneys which we have examined from every clinical variety of the syndrome the disruptive lesion has been found in every portion of the nephron from the origin of the proximal convolution at the glomerulus (Plate 13) to the connecting tubule where it joins the collecting ducts (Plate 4). In no particular clinical variety of kidney have we observed any especial distribution and even in a single kidney there is great variability in its location in the involved nephrons. Moreover, even in severely damaged kiclneys, not all nephrons are affected and in the moderate example, only occasional ones. Randomness of distribution, both in the nephron and among nephrons, is therefore another characteristic of the disruptive lesion.

Although the disruptive lesion is found in all segments of the nephron there are two locations where it is most obvious. The first of these is the terminal portion of the proximal convolution where it dips into the medulla (Plates 3, 6, 14 and 18 ) and the second is the distal convolution and connecting tubule (Plates 4, 5,7 and 10). Actual disruption and fragmentation of the tubule is somewhat less evident in the periglomerular portion of the proximal convolution although it may be found here and often is easily identified in the dissected specimens by means of the peritubular adhesions which surround it (Plates 9 and 26). Short stretches of damaged proximal tubule alternating with well preserved tubule are common enough, however, and produce a patchy irregularity in the appearance of the stained specimen that is in striking contrast to the evenly stained pattern of the normal convolution (Plate 3 ).

In the terminal portion of the proximal convolution the tubular damage is seen in its maximum development: the disintegrating basement membrane exists only in thin, barely perceptible outline and the epithelium is necrotic and desquamated. Long stretches of tubule may thus be denuded of their epithelial lining and as a result are reduced to a third of their original diameter (Plates 3,6 and 14). They thus no longer have the typical appearance of proximal convolutions but resemble damaged limbs, either descending or ascending, of Henle's loop. In such instances, even in the continuity of the dissected nephron, the point of transition to the thin limb of Henle's loop with which they are continuous can be de- 
termined only by the fact that proximal convolutions have a relatively constant length in each species. These terminal portions of the proximal convolution, it will be remembered, lie in the outer stripe of the outer zone of the medulla interspersed among ascending and descending limbs of Henle's loop, a topographical configuration which makes any identification of one from the other in histological section quite impossible (Plates 3 and 7 ).

Although debris and loose conglomerations of desquamated cells may be found in the damaged proximal convolutions, one rarely finds consolidated "casts" as the term is applied to the coagulative plugs that are common in ascending limbs, distal convolutions and collecting tubules of all forms of kidney disease (13).

As might be expected from the differing morphological and functional characteristics of the distal convolution and connecting tubule, tubular disruption in these regions produces lesions of a somewhat different appearance from those seen in the proximal convolution. The damage may involve a short segment or a considerable portion of the affected tubule. Around the point of disruption peritubular adhesions and ingrowths of reactive granulation tissue are frequent and extensive (Plates 5 and 10). A well-formed coagulative cast may or may not be present at the site of rupture; however, since cast formation is frequent in this region of the nephron, many but not all damaged distal convolutions contain such a coagulum (Plate 25). Not infrequently, however, it is found to lie above or below and not contiguous to the point of tubular rupture (Plates 4 and 19).

The disruptive lesion is frequent in both limbs of Henle's loop and may consist of a repetition of short damaged segments or of long stretches that include the greater part of the limb.

To summarize, the distribution of the disruptive lesion in a single nephron is entirely random: there may be severe damage in the proximal convolution and none in the remainder of the nephron, though in such cases the ascending limb and distal convolution are certain to contain much debris and probably casts. In another nephron from the same kidney the distal convolution may show extensive disruption, either with or without cast formation, and the other segments be normal. In another, only the ascending limb of Henle's loop is involved, either in its entirety or in segmental fashion with stretches of well preserved tubule intervening. Nor are all the nephrons affected, for even in severely damaged kidneys a considerable number contain only casts in the ascending limb or distal convolution and the tubule wall is intact throughout.

The disruptive lesion is clearly quite different from the damage that results from the action of toxic agents on renal epithelium and which is commonly considered characteristic of a "nephrosis." In the toxic tubular lesion the renal epithelial cells undergo regressive change, die, desquamate and disintegrate to debris that may be seen filling the space outlined by the basement membrane. This structure suffers no visible change but remains intact and on its supporting surface regenerating epithelial cells may ultimately reconstitute the tubule. These tubular lesions, as will be seen in our next subdivision, are commonly found in certain examples of acute renal failure. Plate $16 \mathrm{~A}$ and $\mathrm{B}^{1}$ show the contrast between disruptive and toxic lesion.

Two sorts of tubular lesion, strikingly different in the nature of the damage that results to the tubule both in regard to structural alteration, location in the nephron and the degree of later regenerative repair that is possible, must therefore be recognized. For these reasons it would seem that they should be distinguished by appropriate terms: nephrotoxic tubular damage describes the one and some such term as tubulorhexis seems appropriate for the disruptive lesion.

\section{Nephrotoxic tubular damage}

If the clinical origin of many of the examples of "lower nephron nephrosis" is considered, it would seem certain that toxic damage to the kidney must be a factor in the development of whatever renal lesion may be present. Such, for example, are the 20 cases of poisoning in Lucké's summary which includes such toxic agents as mushrooms, denatured alcohol, arsenicals, carbon tetrachloride, and phenol.

As we have mentioned in our discussion of the difficulties involved in the concept of "lower nephron nephrosis," the presence of these cases in the syndrome, as its structural status has been de- 
scribed, is a source of considerable perplexity, for all past experimental study of toxic substances indicates that they damage not distal but proximal convolutions (14). Our findings to this point might seem on first thought to have at least cleared this difficulty for we have found that the proximal convolution is as badly damaged in some fashion or other as is the distal in even the classical example of the "crush kidney."

And so it might be thought that at this point in our exposition a major stroke of simplification would be possible: under the general heading of Acute Renal Failure we have only to put the cases of renal poisoning in one group and the kidneys of "pure" shock in another. The first could be the plain or garden variety of "nephrosis," its lesion being that of nephrotoxic damage and the latter, if not "lower nephron," at least some other special brand characterized by the presence of tubulorhextic lesions.

But any such procedure would be to cut, not unravel the pathogenetic tangle for as our dissections will show we are faced with the fact that the examples of renal poisoning have both sorts of tubule lesion, tubulorhextic and nephrotoxic. Lucké was therefore well justified in recognizing common qualities in kidneys from crush injury and carbon tetrachloride poisoning. Moreover the clinical and functional evidences, which we shall consider later, strongly suggest, or one might say demand, a certain degree of unity in the overall group of Acute Renal Failure.

The contrast between the structural changes of tubular damage due to a nephrotoxic agent and those of the tubulorhextic lesion, therefore, still remains as a complication to pathogenetic theory. Its solution requires experimental examination and will therefore be delayed for later consideration. At this time we shall describe typical examples of human kidneys that showed in significant degree nephrotoxic tubular lesions as well as the disruptive tubulorhextic damage.

Again to contrast briefly the two types of tubular damage, the most distinctive difference is found in the lack of the element of disruption of the tubule wall in nephrotoxic damage: the epithelial cells are dead and may ultimately be reduced to granular debris that packs, sausage-like, an intact basement membrane (Plate 16A). The location of the toxic lesions also shows a striking difference from what is observed of tubulorhextic disruption: the former occur only in the proximal convolutions and, moreover, are evenly distributed among all the nephrons.

Our material consisted of 21 human kidneys from instances of definite clinical poisoning. These were supplemented by the kidneys of 29 experimental animals, dogs and rabbits, which were used in our study of the relation of renal ischemia to toxic renal injury which will be described later. In detail, the examples of human kidneys dissected were as follows:

$\begin{array}{lr}\text { Mercuric chloride } & 7 \\ \text { Potassium chlorate } & 1 \\ \text { Di-ethylene glycol } & 2 \\ \text { Mushroom } & 1 \\ \text { Carbon tetrachloride } & 2 \\ \text { Sulfonamides } & 10\end{array}$

It might be objected that kidneys damaged by mercuric chloride should not be included in our list, for although the other forms of renal poisoning that we have given are accepted as proper examples of lower nephron nephrosis, it is generally admitted by those who have supported this concept that corrosive sublimate is the classical example of a toxic agent which damages the "upper" nephron, or, in more accurate terms, the proximal convolution. As we have stated, this fact has been experimentally established $(14,15)$. From what we have seen in our examination of the "crush kidney," the example par excellence of the "lower" nephron nephroses, the acceptance of the "unique" characteristic that presumably establishes this latter syndrome is subject to considerable reservation, for the tubulorhextic lesions were found as frequently in the proximal as in the distal portions of the nephrons. We shall now see that the lesion in the "sublimate kidney" is also less simple than the classical description indicates when it is viewed in the continuity of dissected nephrons from fatal cases of human poisoning. To the resolution of this added complication we shall return at a later time; at present we shall describe without theoretical comment what was found in the kidneys of those who had died of the acute renal failure associated with toxic injury.

A 44 year old woman took six mercuric bichloride tablets $(3.0 \mathrm{gm}$.) and entered the hospital with nausea and abdominal cramps three hours later. Her blood 
pressure was $86 / 60$. Five hours after the ingestion of the poison, BAL treatment was begun and she was given $2 \mathrm{cc}$. every four hours for six days, and then $1 \mathrm{cc}$. every 12 hours for five days. During the first 42 hours the patient had diarrhea, vomited and suffered abdominal cramps. Fifty cc. of urine were passed in the first 24 hours, then none for five days. The urinary output rose slowly for six days to $935 \mathrm{cc}$. and then fell to an average of $120 \mathrm{cc}$. until death. The NPN on the second day was $53 \mathrm{mg}$. per cent rising to 450 on the 28 th day. On the 29th day she died. Clearances of inulin, creatinine and $\mathrm{PAH}$ were infinitesimal on six occasions.

Histological sections showed extreme tubular damage concerning the location of which we need not speculate until our dissections, which accurately determine the seat and nature of the lesions, are described. A most striking histological feature was the interstitial edema and infiltration with monocytes and leucocytes which had occurred throughout the cortex and outer medulla. This reaction was especially intense and at times could be seen to originate around disrupted cross sections of tubules which had the general location of the distal convolution. Extensive regeneration as evidenced by atypical epithelial cells either lining the tubule wall or forming irregular masses that impinged on the tubular lumen could be seen in the sections (Figure 1).

In the dissected nephrons extensive tubular damage of the nephrotoxic type was present in

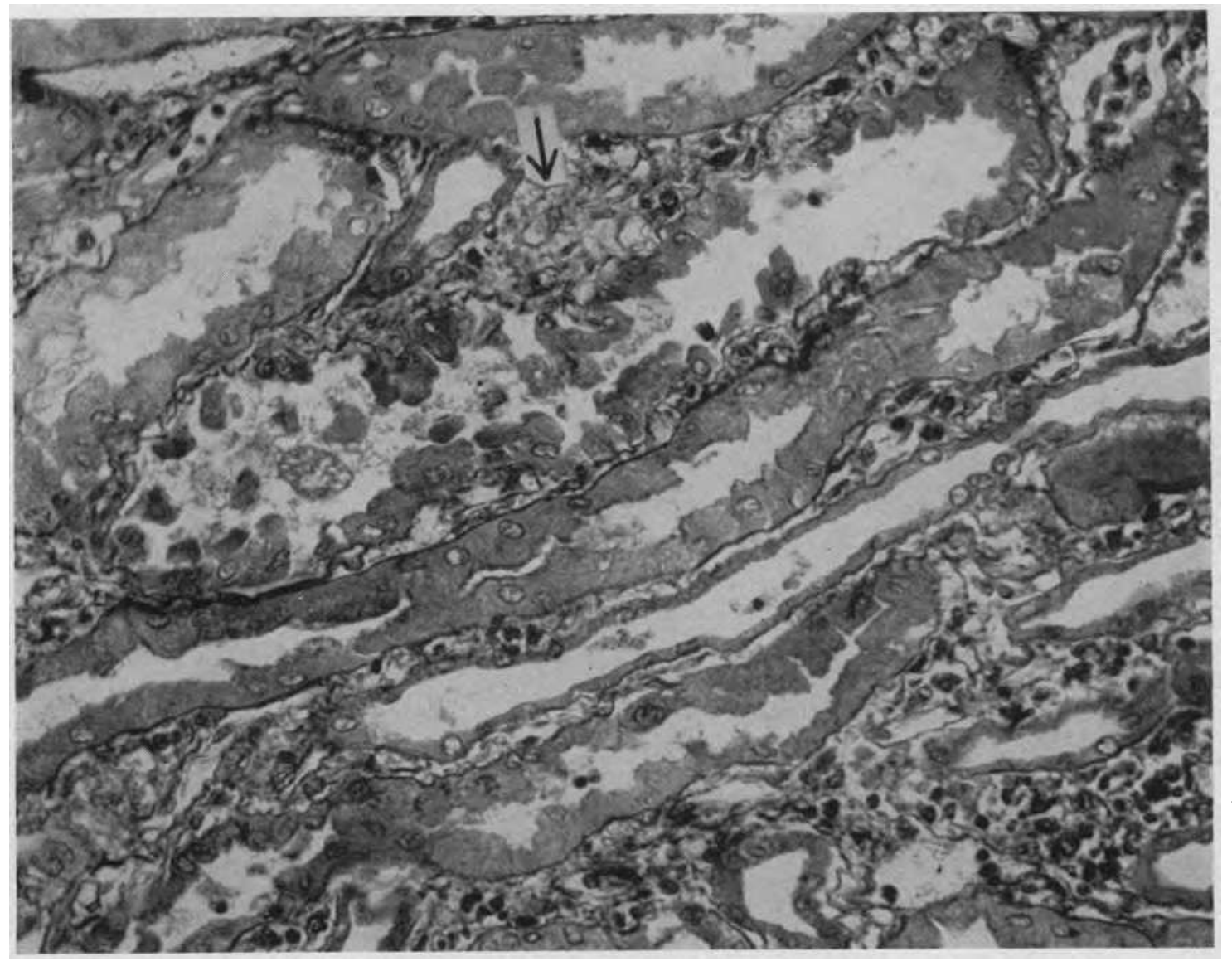

Fig. 1. Sublimate Poisoning-Section through Terminal Segments of Damaged Proximal Convolutions

The normal diameter of these segments is represented by the two lower tubules, the epithelium of which is largely necrotic. Above is a section through a greatly swollen tubule apparently similar to the disruptive lesion shown in Plate $16 \mathrm{~B}^{1}$. Note the heaped up irregular mass of proliferating epithelium and at the arrow, the disruption of the basement membrane. Below to the right the inflammatory infiltration in the interstitial tissue is apparent. Magnification $250 \times$. 
the proximal convolutions: scattered along the course of these damaged convolutions were stretches of disruptive tubulorhexis in which the tubule was greatly swollen, its basement membrane destroyed and the lumen open to the exterior. Plate 16 shows such a lesion in the terminal portion of a proximal convolution; to the upper left and horizontally, the necrotic but still intact tubule can be seen, then follows, on the extreme right, a stretch of disrupted tubule and the convolution ends in the narrowing descending limb, again intact as a tubule but necrotic. The dissection of such nephrons was exceedingly difficult since their continuity had been practically destroyed by the disruptive lesions. In these lesions, masses of proliferating cells could be seen with well stained prominent nuclei. As we shall at a later point return to the reparative processes that occur in damaged kidneys they are only noted here in passing.

Plate 17 shows a collecting tubule (left) and two attached distal convolutions. In the greater part of all, the lumen is filled with a solid cast which stains a deep black with the iron haemotoxylin. In sections, similar casts stained with eosine and gave no reaction for heme pigments or iron; they are therefore simple hyaline or granular casts composed of coagulated protein and cellular debris. The detail in the lowermost of the distal convolutions is largely obscured by this cast formation. The first portion of the uppermost distal convolution has been almost completely relined by a thin layer of atypical epithelial cells; at certain points the disruptions are not completely closed. Further evidence of the incompleteness of tubular restitution is seen in the irregular strictures and dilatations of what was originally a smoothly contoured tubule.

Our five other examples of fatal poisoning with mercuric chloride added little else to the picture of renal damage by this agent. The survival periods were four, eight, 11 and 14 days and one unknown, so that there were individual differences especially in regard to regeneration in the various cases. Of the six, four showed interstitial edema and leucocytic and round cell infiltration to a considerable degree and one only focally around ruptured tubules. The remaining case showed, in conformity with the classical description, no evi- dence of an inflammatory process. In only one case was there calcification of luminal debris.

Another example of the renal lesion associated with toxic injury is the case of a man of 19 admitted for nausea, vomiting, abdominal cramps and black urine of 30 hours duration. Before death he admitted to eating two tubes of tooth paste containing 40 per cent of potassium chlorate. The coffee colored urine showed $4+$ albumin, on admission. The patient was given infusions of 5 per cent glucose and sucrose; vomiting continued to the end. The NPN on the fourth day was $128 \mathrm{mg}$. per cent; on the ninth day, 165 . On the fifth day the urine volume was $80 \mathrm{cc}$. in 24 hours and then rose to $200 \mathrm{cc}$, finally to decrease with death on the 11 th day.

Sections of the kidney showed extensive damage to the tubular epithelium that will be described as it appears in our dissected nephrons; the glomeruli showed no lesion other than evidence of increased permeability to plasma proteins (Figure 2). In the sub-cortical medullary zone, that is, in the region occupied by the terminal segments of the proximal convolution, there was a focal infiltration with round cells, plasma cells and occasional polymorphonuclear leucocytes. As our dissection will show, it was in these terminal segments that the greatest damage had occurred.

Plate 18 is a typical complete proximal convolution. Its first half shows a relatively well preserved although swollen tubule but there is little evidence of a nuclear pattern. The terminal portion down to the origin of the narrow limb of Henle's loop is severely damaged with necrosis, desquamation and disintegration of the tubular epithelium. Nuclear debris and cellular detritus fill the course of the tubule. Other proximal convolutions showed disruptive lesions.

In Plate 19 is shown an example of an ascending limb and distal convolution to its junction with a collecting tubule. The nuclear pattern of the swollen ascending limb can still be recognized. In the distal convolution, horizontally center, is a greatly swollen area of tubular disruption. The basement membrane has disintegrated and proliferating epithelial sheets grow at random with no reconstitution of the tubule wall. A dense cast follows and then another area of disruption.

Our two cases of poisoning with di-ethylene glycol will be but briefly mentioned here as they are to be considered in detail at a later point when the reparative processes that occur in the kidney 


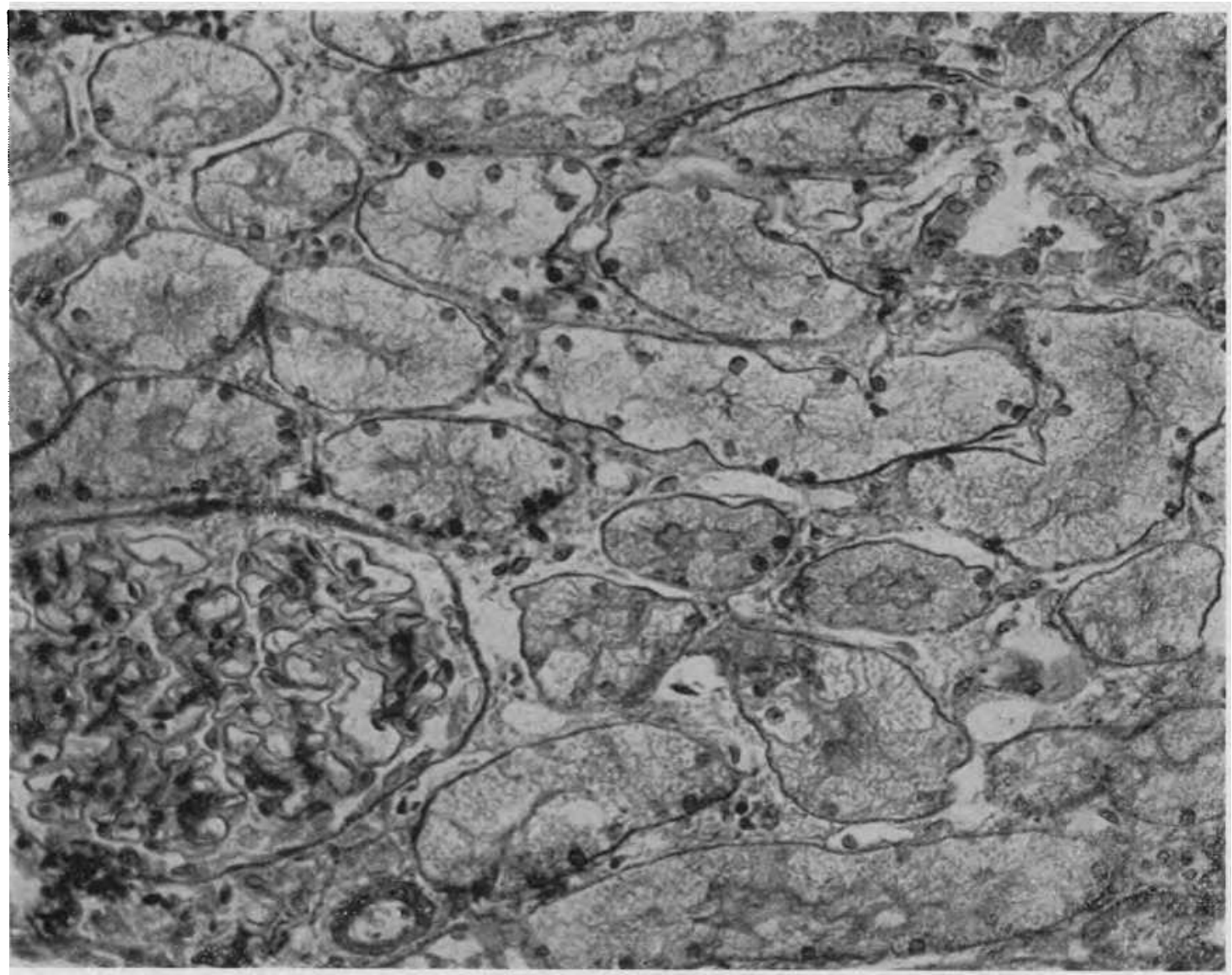

Fig. 2. Potassium Chlorate Poisoning-Section through the Periglomerular loops of a Proximat. Convolution

Some nuclei persist in the swollen vacuolated epithelium but in many, necrosis is complete. The cross sections of this figure correspond to the first half of dissected specimen of Plate 18 . Magnification $250 \times$.

of acute renal failure are described. Sections of the kidneys of two individuals who took the toxic agent at the same time showed on the 12th day the typical vacuolar nephrotoxic lesion in the proximal convolutions. In sections of the kidney from the individual who had survived for three weeks, areas of cellular infiltration with monocytes, plasma cells and occasional polymorphonuclear leucocytes were present in the sub-cortical zone and in foci throughout the cortex. In the dissected specimens there was extensive nephrotoxic damage in the proximal convolutions as well as disruption and dissolution of the basement membrane (Plate 22). In the ascending limbs disruptive lesions were also frequent and more extensive (Plate 23).

One case of mushroom poisoning showed in its proximal convolutions a necrosis of the epithelial cells of its terminal medullary portion as well as frequent tubulorhextic disruptive lesions scattered along the course of the entire nephron from proximal to distal convolution. The same description might be applied to the lesions noted in the nephrons of a case of carbon tetrachloride poisoning. In both cases, focal areas of monocytic infiltration were present in the sub-cortical medulla in the region of tubular damage.

Ten examples of the damage done to the nephron by sulfonamides were examined. The treatment of the tissues with concentrated $\mathrm{HCl}$ had removed all traces of crystalline deposits in our dissected specimens. As has been noted in the literature, considerable variation was seen in different cases in the detail of the renal lesions. 
All showed in histological sections an inflammatory interstitial reaction, either diffusely in the cortex or sub-cortical medulla or in a granulomatous aspect around ruptured tubules. It was the rare example which showed even in histological section the presence of crystals. No special means had been taken in the preparation of this material to preserve these structures, but it seems unlikely that they could have been present in every ruptured tubule, and several of the few that were found lay in tubule cross sections which showed no damage. With no very positive evidence in the matter, but with the knowledge that similar disruptions must have another origin in the other forms of renal toxic damage, we are therefore somewhat sceptical towards the suggestion that rupture of tubules in sulfonamide poisoning is the result of perforation of the wall by crystals.

As has also been the experience of others, we have found considerable variation in the types of damage to the tubule of the nephron in our dissected specimens. In all ten cases definite disruptive lesions were found throughout their entire length from proximal convolution, especially in its terminal medullary segment, to the connecting tubule. In four of the ten cases there was frank necrosis of the middle and terminal portions of the proximal convolution. In Plate 20 is shown a complete example: the tubule is swollen and the nuclear pattern is obscure throughout. In several stretches the disintegration of the tubular epithelium is evident and in its terminal portion it is frankly necrotic.

In the other six cases the nephrotoxic lesion in the proximal convolution was, if present, so slight as to be uncertain. As we have mentioned in the description of our methods and as will be apparent in the discussion of our results, human autopsy material is unsuited even under the best of conditions for the determination of slight epithelial alterations, for both in histological section and dissected specimen the demonstration of the finer cytoplasmic detail, such as the condition of the mitochondrial rodlets, requires immediate fixation.

In Plate 21 an ascending limb, left lower, with a well preserved nuclear pattern passes up to a distal convolution in which there are several disruptive lesions. An attempt at regenerative repair may be noted but the break in the tubule wall is not closed. Another distal convolution joins from above and the collecting tubule, arching downward and then up to the right, is filled with irregular masses of detritus and debris, but its cellular pattern is perfectly preserved.

Later in our exposition we shall return to a further consideration of the damage that is found in kidneys of acute renal failure associated with toxic injury, first when the problem of repair in the damaged kidney is discussed and later when an experimental examination of the problem of the aetiological relations of the two sorts of tubular lesion, nephrotoxic and tubulorhextic, is examined. Considerably more data will thereby be available concerning renal toxic injury.

For the present, two points may be emphasized. First, all examples of fatal acute renal failure due to poisoning that we have examined have shown not only the classical nephrotoxic necrotizing lesion in the proximal convolution but also, scattered throughout the length of the nephron, the disruptive tubulorhextic lesion that was the prevalent feature in the kidney of crush injury.

The second is the regular occurrence of an interstitial inflammatory reaction in greater or less degree in fatal acute renal failure whether it had arisen on the clinical basis of traumatic or toxic injury. This reaction was particularly prominent in the sub-cortical medulla where the terminal portions of the proximal convolution lie which, as our dissections have shown, are the frequent sites of disruptive tubular damage: it was also prominent in histological sections in the cortex around disrupted distal tubules.

These interstitial reactions have been generally recognized as an integral aspect of the renal lesion in certain of the "lower nephron nephroses," such as the crush and sulfonamide kidney, but at least not emphasized or even mentioned in other members of the group of frank toxic origin. This perhaps stems from the subconscious feeling that the essential toxic lesion, following sublimate for example, must be "non-inflammatory," a fact firmly established indeed by extensive experimental evidence. We see again, therefore, the semantic, yet nonetheless real confusion that has resulted from the introduction of the term "nephrosis" and its variant of the "lower nephron" 
into the nomenclature of renal disease. It is not surprising that $\mathrm{A}$. and $\mathrm{H}$. Bergstrand object most strongly to the "-osis," and insist quite logically that it must be an "-itis" (16), for originally (17), and to many authorities today, this is the distinction for which the etymological curiosity was coined. Since, as we have said elsewhere, "nephrosis" in its protean connotations is "all things to all men," it is doubtless futile to insist on any particular meaning in the use of the term. Leaving the solution of the semantic aspect of the problem to the ages, we can at least offer an explanation of the apparently incongruous fact that the damage of fatal acute renal failure due to a toxic injury that is characterized by the necrotizing, non-inflammatory tubular lesion also shows an inflammatory element in the interstitial tissue of the kidney. The latter is obviously not the result of the toxic action which caused necrosis of the epithelium of the proximal convolution but, as can be seen from its localization, is related to the disruptive tubulorhextic lesion. The "cause" of the interstitial reaction is therefore tied to the aetiological factors that operate in the production of tubular disruption and these will be examined by experimental methods at a later time.

\section{The collecting tubules and cast formation}

No damage has been found in the collecting tubules of any of the kidneys of Acute Renal Failure save compression of epithelial cells and occasional desquamation; these ducts, however, contain casts and debris in proportion to the amount of damage that has occurred in their contiguous nephrons. These occlusions are essentially similar in their morphological aspects to those seen in all forms of renal damage (13), but a description of them as they occur in the kidney of Acute Renal Failure may be repeated, as the appearance of the tubular "cast" is strikingly different in dissected material from what is seen in the conventional histological section. Functional implications drawn, as they usually are, from the latter are apt to be inadequate. Some casts, when seen in their entirety lying in situ in the upper or middle branchings of the collecting system, are relatively short plugs and their appearance is not very different from what is observed in longitudinal histological sections of a collecting tubule (Plates 17, 21 and 25). Many other coagula are of relatively enormous extent: they may be traced as a continuous structure in dissected specimens from their origin in the ascending limb of a nephron, through its distal and connecting tubule into the peripheral branch of the collecting tubule and then down through the cortex to a point deep in the medulla. In its course through the cortex are found branch-like extensions of solid material çontinuous from other damaged nephrons which drained into the occluded collecting duct, so that the whole coagulum forms a hydra-like be-tentacled structure 2 to 3 $\mathrm{cm}$. in length (Plates 4 and 17). Yet in a histological section such a complex object might appear as a few scattered sections of "tubules and casts" that bear no apparent topographical relation to each other.

These ramifying coagula are not homogeneous in structure throughout their considerable length. In some, long stretches are composed of clear hyaline material and in this substance are irregular collections of granular deposit either diffusely scattered or conglomerated in irregular masses. The dark staining granular material is evidently in part cellular debris, for fragments of epithelial cells are at times recognizable (Plate 23). Pigments and crystals, to be described more fully later, are also present. In other examples the entire extent of the coagulum may be opaque and granular from an admixture of heterogeneous debris.

\section{Pigment deposition in intratubular debris and casts}

In considering the appearance of the heme pigments in our preparations it should be recalled that the kidney tissues had been macerated in concentrated $\mathrm{HCl}$ for 24 to 48 hours preliminary to dissection and staining of the nephrons. This treatment might be expected to alter both the physical and chemical nature of blood pigments but, as will be apparent, our findings in the dissected material agree in all essentials with previous observations made on fixed histological material.

As has been the experience of other investigators, we have found heme pigment in all of the kidneys that we have examined from cases where myoglobin or haemoglobin was liberated into the blood stream as part of the primary cause of the clinical disturbance. In these and many other instances blood transfusions had been given and though a 
transfusion reaction may not have been noted in the clinical record an added source of the products of blood degradation was therefore present. In the cases in which there was no evidence in the clinical record that haemolysis or muscle injury had occurred the typical disruptive tubular lesions were found with no histochemical evidence, such as the benzidine or Prussian Blue test, of heme pigment in casts or tubular debris. It is noteworthy, therefore, that in this group of constant and similar renal disturbances associated with shock and related conditions of Acute Renal Failure there is from their very origin no constancy in regard to any aetiological factor that concerns heme prgmentation.

The appearance of the pigment in the dissected nephrons differs as it is seen in fully formed casts, i.e., in the upper ascending limbs of Henle's loop, the distal convolutions and the collecting tubules, or in the loose unconsolidated deloris that is found in the proximal convolution.

The appearance of the heme pigments in casts as they lie in situ in the lumen of the dissected nephrons is essentially that which one would expect to see when viewing in their entirety the objects that have previously been described in histological section. What would otherwise be designated as a "hyaline" cast may show an evenly diffused, faint brownish discoloration in the unstained specimen. When stained with iron haemotoxylin these coagula take on a dense black color, the same reaction that is seen in them and in red blood cells in histological sections. When tested for free iron by the Berlin Blue method the casts in distal portions of the nephrons and in the collecting tubules are negative, but are positive to the benzidine reaction. The treatment with $\mathrm{HCl}$ has therefore not affected these histochemical reactions. In long casts that run continuously through considerable lengths of tubule it is often observed that the concentration of pigment varies greatly and at times with remarkable abruptness in various portions of the coagulum. Histological sections of such a structure would be interpreted as several separate and different sorts of casts.

In other examples the heme pigment is less evenly and finely dispersed in the substance of the matrix so that a definitely granular appearance is produced which may be exaggerated to the extent that conglomerates or sphericles of pigment and, in the extreme case, deformed crystalline deposits are seen. We have never observed crystalline deposits unassociated with organic matrix. In restricted stretches of tubule this appearance may be simulated but by following along the tubule it becomes evident that these apparently isolated "free" crystals form a part of a coagulated structure.

The general distribution and optical characteristics of the heme pigment in the casts in the isolated nephrons have therefore the appearance that might be expected if a column of protein-containing fluid with irregularly distributed concentrations of heme pigment had coagulated with the production of a varying and discontinuous pattern of pigment deposit, from a molecularly dispersed, diffuse staining to the inclusion of relatively pure crystalline masses.

As was the case in our correlation of the presence of casts with tubular disruption, no positive result was observed between the presence of pigment deposition, either free or in casts, with tubular lesions. If a cast was fortuitously present at the point of rupture, it often but not always contained heme pigment. but other casts of great extent filling continuously the ascending limb and distal convolution with extreme concentrations of granular pigments were commonly observed surrounded by an intact and well preserved tubular wall (Plates 4, 7, 10,12,15 and 19). There was therefore no evidence that the presence of large concentrations of heme pigment had any specific ill-effect on the tubular epithelium.

We have found little mention in previous studies of shock kiclneys of the occurrence of heme pigments in the proximal convolutions. This seems remarkable for much experimental work has shown that under normal circumstances these pigments are selectively absorbed, stored and metabolized, as are other proteins (9). by the epithelial cells of this portion of the nephron (18). The physiological conditions under which heme pigments occur in the proximal convolution are therefore very different from what obtains in the distal nephron where the pigment lies in the tubule lumen unabsorbed and unchanged, as is shown by the positive benzidine and negative Berlin Blue tests, by the activity of the renal epithelium. Our findings in both sections and in dissected nephrons of human kidneys were difficult of interpretation 
until we had studied these processes in the kidneys of experimental shock. Our description and discussion of heme pigments in the proximal convolution will therefore be deferred until the latter have been described.

\section{Reparative processes in the kidney of Acute Renal Failure}

Whereas the clinician has opportunities to follow the restoration of function in the damaged kidney the pathologist sees only the more extreme of renal lesions and only those that did not recover. His factual knowledge is therefore confined to what he sees of inadequate attempts at restitution of a normal structural and functional status in the damaged kidney; to this he commonly appends by a sort of imaginative extrapolation a description of what he thinks the course of adequate repair must be in those conditions of lesser damage of which he has no first-hand experience. The resulting descriptions of what is called "tubular regeneration" are perhaps adequate for concepts of renal structure based solely on the study of histological sections which describe the kidney as composed of "glomeruli" and various sorts of "tubules," but they prove quite meaningless in considering the repair of a tortuous and tenuous organ like the nephron where continuity is essential to normal function. Failure of restitution of a freely draining lumen at a single point in its course, for example, or the persistence of a single minute hole in its wall, may destroy the total functional integrity of the whole organ so far as its excretory capacity is concerned, no matter how many regenerating epithelial cells may have been seen by the observer's imaginative eye to be "creeping beneath the dead debris to reline the most damaged areas."

A consideration of the appearance of the disruptive lesion in Plates 5, 10 and 16 will show at a glance that its most severe form is probably irreparable. Disruption or dissolution of the basement membrane, the characteristic that distinguishes this lesion from that of nephrotoxic damage, is the factor which prevents repair, for not only is there no directing surface on which regenerating epithelial cells "creep" to reform the tubule, but the gap in the tubule wall is invaded by granulation tissue which fills and obliterates the tubule lumen. Here again, then, is seen the antithesis of tubular disruption and the tubular lesion of nephrotoxic damage, for in the latter, as exemplified by the kidney of the moderate example of sublimate poisoning at least in its experimental form, regenerating epithelium relines the bare but intact membrana propria so that both structure and function are restored.

It may be that minor disruptions of the tubule wall are plugged by proliferating cells; certainly evidence of epithelial regeneration, as contrasted to nephron reconstitution, is frequently found in the dissected specimens. At the margin of the tubular disruption may be seen large atypical epithelial cells with hyperchromatic nuclei, an example of exuberant but obviously futile regeneration, and analogous abortive attempts at repair, as evidenced by an irregular heaping up of newformed but misplaced cells, are shown in Plates $16,17,19,21,24$ and 25.

In instances where considerable amounts of blood pigment have flowed through damaged tubules, as in the kidney of the transfusion accident, it can also be seen that the regenerating renal epithelium has regained at least certain of its functions. In Plate 12 long stretches of damaged proximal corvolution have been relined with an atypical epithelium containing irregularly distributed, oval nuclei, yet in spite of this structural abnormality the cells have absorbed blood pigment and reduced it in part to hemosiderin. Yet in spite of these good intentions no restitution of the function of the nephron as an excretory organ has occurred when the distal convolution, as shown in Plates 5, 10, 19, 23 and 25, is the seat of an irreparable disruption.

Recovery of a structural renal status compatible with adequate function therefore becomes a complex matter when the problem is considered, as it must be, from the viewpoint of "restitution of nephrons" and not "regeneration of the kidney tubules" (19). The detail of three clinical cases may be given to illustrate the varied course of the reparative processes.

The first two examples form an almost perfect experimental examination of the problem of repair in acute renal failure related to toxic injury. We are indebted to Drs. E. S. Breed and C. F. Baxter for their functional data which they will publish in detail at a later time, to Dr. R. S. Hotchkiss for 


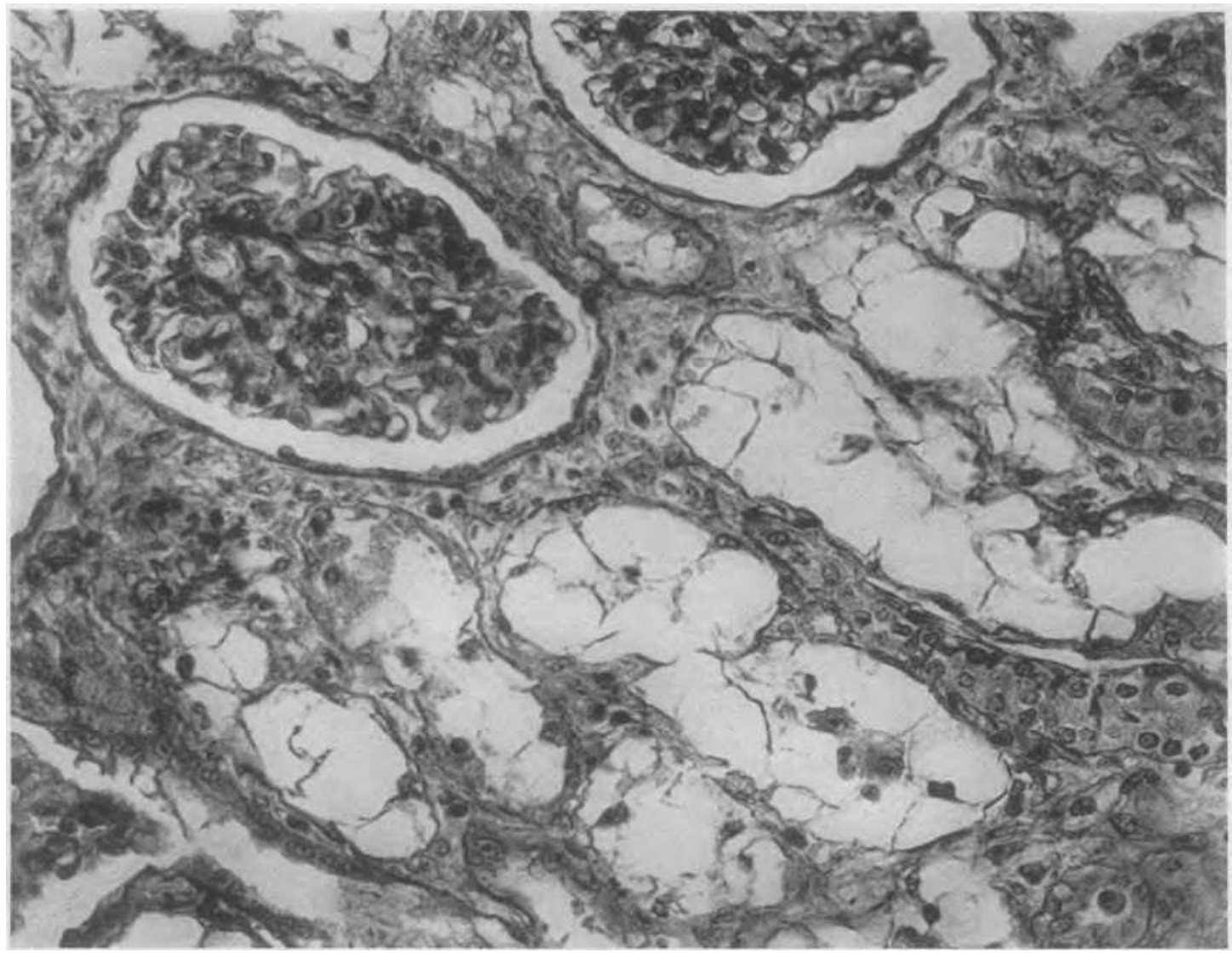

Fig. 3. Di-Ethylene Glycol Poisoning-Section of Kidney of W. R. Who Died on the 12th Day

Cross sections of periglomerular coils of proximal convolution showing complete vacuolar necrosis of the renal epithelium. There is no regeneration or significant interstitial inflammatory reaction. Cf. Plate 22 for dissected specimen; also Plate 44. Magnification $250 \times$.

the clinical details and to Dr. Milton Helpern for the anatomical material.

Two young, apparently healthy prisoners, W.R. and D., in a penal institution obtained surreptitiously a supply of what was described as an anti-freeze mixture. How much was drunk and for how long continued is not known but after eight days W. R. was admitted to the hospital with bilateral kidney pain and shortness of breath; red cells and albumin were found in his urine. His blood pressure was 170/100. On the 12th day his PAH, $\mathrm{O}_{2}$ and inulin extraction ratios were all 0 ; the BUN was $266 \mathrm{mg}$. per cent During four hours $0.02 \mathrm{cc} . / \mathrm{min}$. of urine was collected. The blood $\mathrm{K}$ was $8.85 \mathrm{~m} . \mathrm{eq} . / 1$. Decapsulation was attempted during which operation he died of cardiac arrest.

D. came to the hospital on the day following his companion's entrance with essentially similar clinical findings. On the 11th day his urine flow was $0.035 \mathrm{cc} . / \mathrm{min}$. An "artificial kidney" reduced his blood pressure from 200/80 to $110 / 70$, his NPN from 300 to $180 \mathrm{mg}$. per cent., and his blood $\mathrm{K}$ from 8 to $5.19 \mathrm{~m} . \mathrm{eq} / 1$. The next day the ex- traction ratios of $\mathrm{PAH}$, inulin and $\mathrm{O}_{2}$ were 0 . That afternoon a decapsulation was done and a biopsy obtained from the right kidney. The patient did fairly well postoperatively; on the 16th day he was put on the artificial kidney again and his blood $\mathrm{K}$ reduced from 8.6 to 5.5 m.eq/1. At this time NPN was $324 \mathrm{mg}$. per cent. The urine output increased gradually from $89 \mathrm{cc}$. in 24 hours on the 17 th day to $1151 \mathrm{cc}$. on the $21 \mathrm{st}$ day. His inulin clearance was then $1.92 \mathrm{cc} . / \mathrm{min}$., creatinine $1.16 / \mathrm{min}$. and PAH $1.31 / \mathrm{min}$. Three days later he became disoriented and died.

The kidneys were therefore available from two individuals who took the same nephrotoxic agent, di-ethylene glycol as subsequent histological examination proved, at the same time. One (W. R.) died on the 12 th day at what might be described as the height of renal damage and in renal failure. A biopsy was taken from the other (D.) at approximately the same period after the poison- 
ing, thus allowing a comparison of the anatomical lesion in the kidneys of the two cases at the point of its maximum development. The individual, D., showed a functional recovery so far as water excretion was concerned, but died of renal failure 24 days after taking the poison.

Histological sections of the kidney of W. R. who died on the 12th day showed the typical vacuolar epithelial alteration caused by di-ethylene glycol in sections of what are apparently proximal convolutions; there were casts and debris and other alterations in the other portions of the nephron. There was no evidence of epithelial regeneration (Figure 3).

Dissections of nephrons showed the full development of the renal lesion with no evidence of repair. In Plate 22 is shown the glomerulus and the first portion of a proximal convolution; the epithelium of the tubule is completely disarranged; the vacuolar change is not evident in the thickness of the dissected specimen which was purposely heavily stained in order that the protoplasmic remnants of the epithelium would be visible. The dissected specimen shows quite clearly that the damage is more than a nephrotoxic epithelial lesion, for the basement membrane has been destroyed along the greater part of the preparation and at several places in the course of the tubule typical disruptive tubulorhexis is evident. In ascending limbs and distal convolutions (Plate 23) the disruptive tubular lesions are also present along with casts and diffuse basement membrane damage. The epithelium of the collecting tubule, however, is intact though its lumen contains irregular collec-

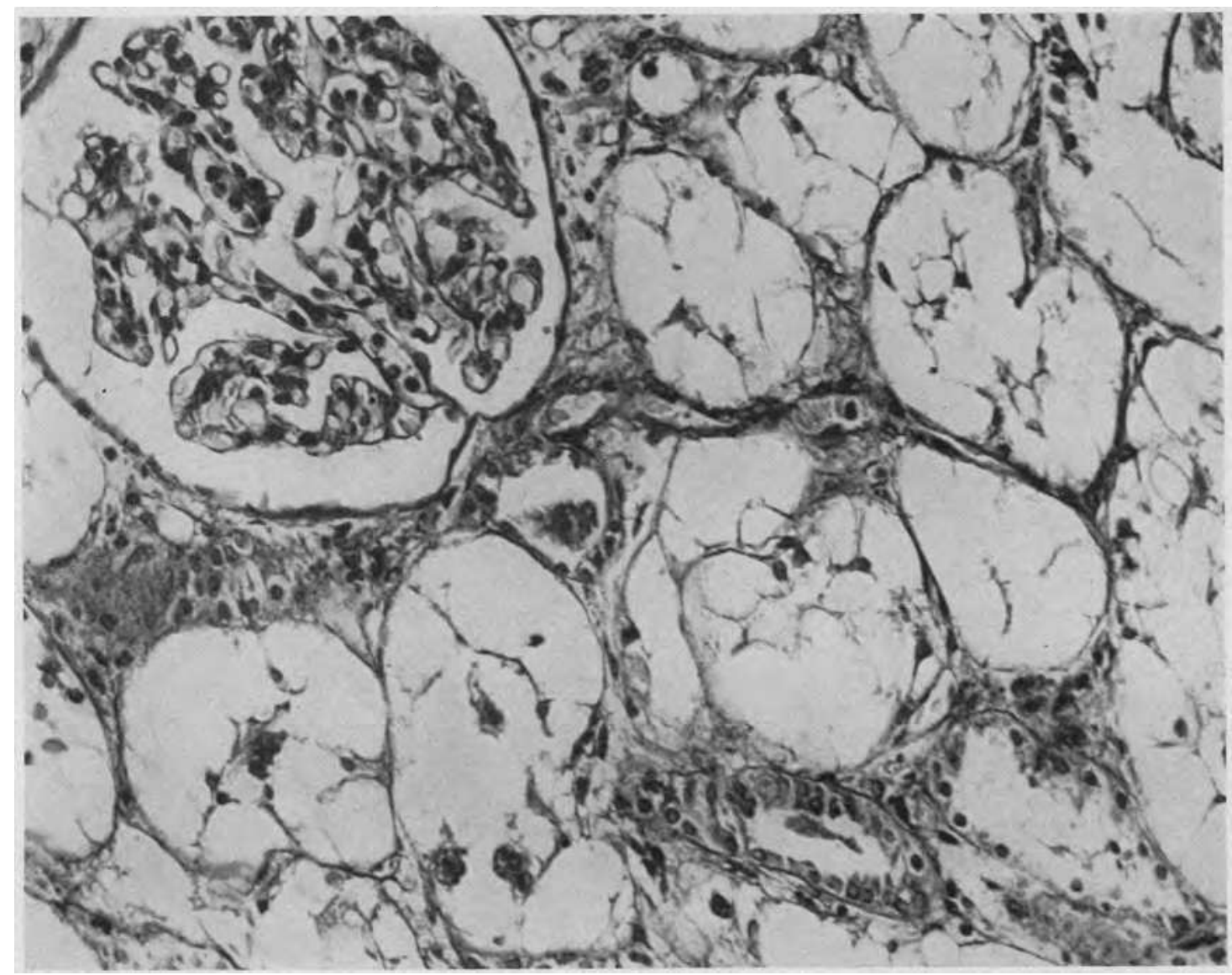

Fig. 4. Di-Ethylene Glycol Poisoning-Biopsy from the Cortex of The Kidney of D. on the 11th DAY

Note that the lesion is identical with that of the other case shown in Figure 3. Magnification $250 \times$. 


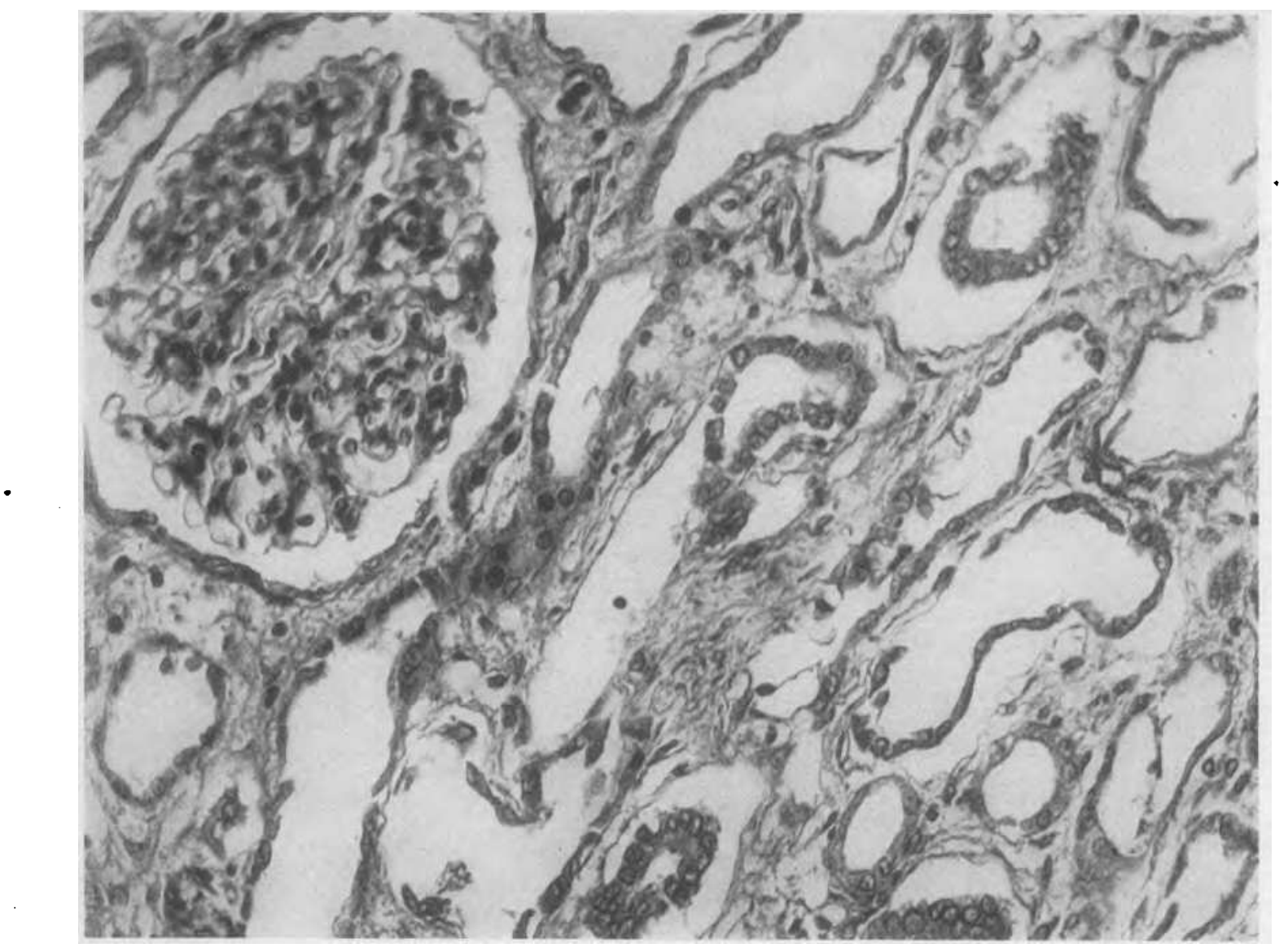

Fig. 5. Di-Ethylene Glycol Poisoning-The Kidney of D. at Autopsy 13 Days after the Taking of the Biopsy of the Preceding Figure

No vacuolated necrotic cells remain, the original tubular lining being completely replaced by the proliferation of a flat epithelium. There was, however, no significant return of tubular function and the lack of restitution of a nephron structure compatible with function is seen in Plates 24 and 25. Magnification $250 \times$.

tions of cellular debris and casts. The over-all picture of the renal lesion may therefore by summarized as a combination of extensive nephrotoxic and disruptive tubular damage with no regeneration or repair.

Histological sections of the biopsy taken on the 11 th day from the kidney of $D$. showed widespread and acute lesions identical to those observed in W. R.; this observation is of importance as it shows the degree of damage in the kidney of this individual 13 days before his death (Figure 4). Histological sections taken from the kidney post mortem, that is, approximately three weeks after the ingestion of the poison, showed none of the acutely damaged vacuolated cells seen in the biopsy in the cross sections of proximal convolutions. These tubules were considerably dilated and completely lined with a flat, atypical, regenerating epithelium which in appearance had reformed the tubule wall. In cross sections of other portions of the nephron, tubular damage and casts were frequent. In the sub-cortical region and extending deep into the medulla there was a heavy infiltration with inflammatory round cells and leucocytes (Figures 5 and 6$)$.

Dissections of proximal convolutions present an entirely different aspect of the epithelial regeneration from what was apparent in histological section, for it can be seen at a glance that the tubule wall is neither reformed nor a continuous 
lumen reestablished, which is the only sort of lumen that has functional significance. The tubule is greatly dilated and its contours are remarkably irregular. This irregularity is due both to sudden constrictions (A) scattered along its length and to sudden fingerlike pouches and protrusions (B) which are formed of dark staining bands and strips of regenerating renal epithelium. Moreover regenerating cells are not confined to the wall of the tubule, but grow in random whorls that double back into the lumen (C) and at times form valve-like flaps and irregular masses which impinge upon and obstruct it (Plate 24).

In the ascending limbs and distal convolutions there is also extensive disruptive tubular damage, extensive cast formation and irregular repair. The sheets and bands of dark staining regenerating epithelium are clearly seen; it will be noticed that they are exceedingly patchy in distribution and that the tubule wall, even where regeneration is most active, is far from reestablished. The dark black segments are portions of relatively intact tubule filled with casts. Frank disruptions of the tubule wall with surrounding ingrowths of inflammatory granulation tissue are present (Plate 25).

To summarize, it is clear that in this kidney which showed grave structural damage on the 11th day, and of which, from the appearances noted in histological section, it would have been said that extensive "tubular regeneration" had occurred by the 24th day, there was little evidence of an adequate reconstitution of functional nephrons in spite of the exuberance of epithelial regeneration.

Referring back to the examples of toxic renal injury due to sublimate and potassium chlorate,

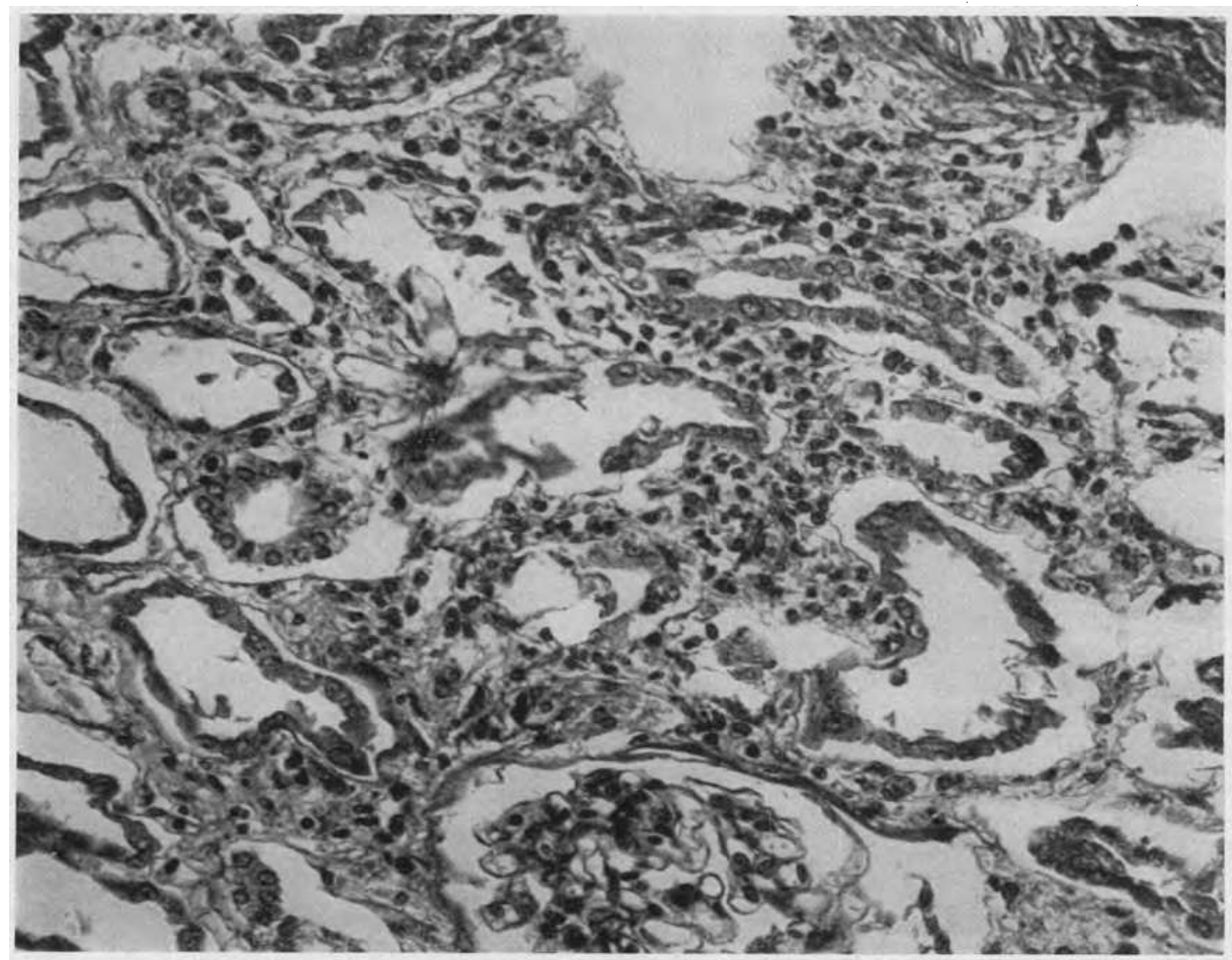

Fig. 6. Same Section as Figure 5 Showing Cross Sections of Disrupted Tubules with Heavy Inflammatory Reaction in the Interstitial Tissue. Magnification $250 \times$ 
similar difficulties in the repair of the disruptive tubular lesions may be noted. In Plate 16 the large disruption in the proximal convolution is filled with masses of dark staining, atypical epithelial cells that protrude into the irregularly dilated lumen and, as a result of the disintegration of the basement membrane, form isolated islands of proliferating epithelium rather than a reconstituted tubule wall. In Plate 19 the swollen disruption in the distal convolution is only partially lined by sheets of epithelium, and these, lacking the support of a basement membrane, stream out irregularly into the tubule lumen.

As evidence of the permanency of the damage that is inflicted on the individual nephrons by the disruptive tubular lesion, an example of repeated episodes of renal damage over a period of years may be cited. Our material was obtained from a case of paroxysmal hemoglobinuria reported by Drs. Ralph Sussman and Herbert Kayden (20). After 11 typical attacks over a period of two years, an extraordinarily severe seizure resulted in oliguria and nitrogen retention with death on the 12th day. The typical lesions of tubular disruption and heme pigment casts were described by Dr. Alfred Plaut and the authors point out the similarity of the renal condition to "post-transfusion and crush injury nephrosis." Of interest to our immediate problem, the histological examination by Dr. Plaut showed not only typical acute tubular lesions but also "a diffuse fibrotic process with numerous scars ... apparently remnants of renal injury resulting from 11 previous hemolytic episodes. It should be noted that no vascular changes were reported to suggest a vascular cause for the renal lesions."

In the more dense fibrotic scars which were scattered through the renal cortex only remnants of nephrons could be found by dissection. These consisted of atrophied bits of what could still be recognized as proximal convolutions attached to shrunken, fibrous glomeruli. In the looser fibrous tissue at the periphery of the scars better preserved but markedly deformed nephrons were found. Plate 26 shows an irregularly atrophic proximal convolution in which are seen several tubulorhextic disruptions surrounded by adhesions of granulation tissue that resulted from the terminal acute attack. In Plate 27 is seen a badly damaged ascending limb and distal con- volution which, probably as a result of peripheral obstruction, shows a huge irregular cystic dilation. Numerous diverticula, the origin of which bears no relation to the renal lesions, are present."

To summarize our findings regarding the reparative process : restitution of function in kidneys of Acute Renal Failure associated with traumatic and toxic injury would seem to depend chiefly on the recovery of functional normality in nephrons which have never suffered the fully developed disruptive lesion. In the case of poisoning, where nephrotoxic tubular lesions with an intact basement membrane predominate, epithelial regeneration can reconstitute the tubule of the nephron with relative ease; if there is extensive tubulorhextic clamage, as there is in fatal poisoning, the reparative problem is less simple. Then, as in the case of "the crush" or "shock" kidney where disruptive tubular lesions prevail, recovery of renal function depends on the restitution of functional normality in nephrons which have never suffered the full brunt of the disruptive injury. The peculiarly spotty distribution of these lesions among the nephrons, some of which show no significant damage in the most grievously altered kidney, makes this eventuality possible.

\section{Summary of the structural alterations in human kidneys in Acute Renal Failure}

Before proceeding to an examination by the experimental method of aetiological problems, a brief summary of the structural alterations seen in the human kidney in Acute Renal Failure related to traumatic and toxic injury should be useful to fix clearly in mind what sort of lesion is to be reproduced.

The distinctive disruption of tubules described by Dunn, Gillespie and Niven is characteristic of all members of this group. This lesion, for which the name tubulorhexis has been suggested, occurs in all parts of nephron indiscriminately from the

${ }^{3}$ These objects, found not infrequently in normal kidneys and only on the distal convolution, may become so large as to have the configuration of glomeruli ( $c f$. insert, Plate 27); they have on occasion been described as such in dissected specimens and so led to much perplexity as to how glomeruli could become attached to collecting tubules. In histological sections they may appear as gland-like structures independent of the tubules and, with their contained colloid-like material, have been interpreted as renal "endocrine" elements. 
proximal convolution to the collecting tubule. Its distribution is entirely random both in the nephron and among the nephrons; even in the kidney that has failed some nephrons are found essentially undamaged. The lesions show no constant association with either casts or accumulations of heme pigment; the lumen of the distal nephron commonly contains these materials with no damage to the tubule wall.

In kidneys from cases where poisoning has been the clinical cause of renal failure there is also found besides the disruptive lesions tubular damage of a nephrotoxic nature; this is located in proximal convolutions and is evenly distributed throughout all the nephrons. Such lesions are absent or minimal in cases where the renal damage follows shock resulting from traumatic injury.

\section{THE REPRODUCTION OF THE LESIONS OF THE KIDNEY OF SHOCK BY EXPERIMENTAL PROCEDURES}

Now that the lesions observed in the human kidney of shock have been described we may return to our earlier stated problems of aetiology, pathogenesis and clinical correlation. It is clear at once, however, that these questions present themselves in a very different form when they arise from the consideration of the description of the structural status of the damaged kidney that we have given rather than on the basis of the current designation of the lesion as a "lower nephron nephrosis." The problems to be put to experimental test are therefore quite different from those which have been examined by previous investigators, and this is particularly true when the experiment is directed toward the reproduction of the human type of lesion in the kidney of animals. $A$ priori, one could be reasonably certain that no such disturbance as an induced ischemia could produce lesions limited to the "lower nephron" for the anatomical characteristics of the renal circulation and the topographical arrangements of the nephron make such an eventuality impossible. As has been suggested in our introduction, the frustrations and difficulties of experimenters in the past were, therefore, due to the fact that questions were asked that had no answer. With the knowledge that proximal convolutions must be damaged if the human lesion is to be reproduced and that in fact any or all parts of the nephron may be involved, we can approach the experimental verification of the thesis of ischemic damage with a new and more hopeful attitude. This is particularly true since there would seem to be little chance that experiments based on a hypothesis of toxic damage would be successful, for it is apparent from our descriptions that the essential disruptive tubular lesion of the human kidney is different, in both its nature and location in the nephron and in its distribution among the nephrons, from what is produced experimentally by nephrotoxic poisons. No toxic substance reaching the kidney by the blood stream could conceivably cause such random damage as we have described in such functionally disparate parts of so many independent organs.

Among all the factors that have been suggested as a cause of the renal lesion, ischemia stands out as the disturbance par excellence which produces random and scattered damage. This is especially so when the circulation fails in small, widely distributed, terminal arteries and arterioles; an example of its effect is seen in the kidney itself where arteriolar-sclerotic fibrous scars, irregularly scattered through the renal cortex, encroach upon and surround atrophied portions of the nephrons at random. So close is this analogy drawn from his general experience, that the pathological anatomist might have little hesitation in deciding our problem on the basis of induction from his structural observations of the human renal lesions alone if the experimental verification of this conclusion were not possible.

As a matter of fact the recent development of a visual technique has made it possible for the morphologist to observe directly the circulatory status of the kidney and, as is desired in our problem, to actually see the pattern of an ischemia as it existed in the experimental animal at the moment prior to its sacrifice. Schlegel and Moses (21) have shown that the fluorescent dye, thioflavin $\mathrm{S}$, when injected intravenously in small amount, diffuses into the wall of the arteries and arterioles and glomeruli and is there retained for a sufficient time so that if the kidney is promptly removed and viewed at low magnification under ultra-violet light all the glomeruli and vessels which had been receiving blood containing the dye stand out in brilliant secondary fluorescence against the darker background of the renal tissue. We 
shall use this technique at a later point in our investigation and will at that time describe more fully the technical details. For the present, one of Drs. Schlegel's and Moses' original illustrations of a normal kidney of a rabbit showing the arteries, arterioles and glomeruli that were receiving blood at the moment of its death is seen in Figure $7 \mathrm{~A}$. The even distribution of the circulatory pattern is most beautifully demonstrated and in contrast, Figure $7 \mathrm{~B}$, taken from the same report, shows the pattern of the renal ischemia that followed the intravenous injection of $1 / 10,000$ adrenalin. Irregular areas of renal cortex are seen in which the patent and fluorescent vessels and glomeruli stand out in brilliant contrast to the patches of dark ischemic tissue where the circulatory bed had received no dye. If one visualizes the distribution of the constituent nephrons on this spotty background of ischemic cortex one can anticipate how the random pattern of tubular clamage that we have described in the nephrons of the human kidneys of shock might develop. (Figure 8.)

Our examination of the structural lesions in the kidney of experimental shock was made on material from the extensive studies of Drs. D. D. Van Slyke, R. A. Phillips. V. P. Dole, P. B. Hamilton, K. Emerson, Jr. and R. M. Archibald, on the functional disturbances that occur in the kidney in this condition $(5,22)$.

In these experiments on dogs the functional effects of the renal ischemia which is known to be part of the general phenomena of shock in man were examined under varying conditions. To determine the effect of cluration of the ischemia. clamps were placed on the renal artery so that blood flow to the kidney was arrested for periods up to three hours and longer. In animals whose renal artery was clamped three hours, the kidneys after a few days of renal inadequacy ultimately recovered their functional capacity; those whose artery was clamped longer died in renal failure. Analogous functional disturbances were observed in dogs in shock when the renal blood flow, estimated by hippurate clearance, had been reduced as a result of a hemorrhage or trauma below 1 per cent of its initial normal rate for a period of four hours. These investigators found that in general the progressive effects of ischemia on the kidney, both in the experimental animals and in human cases, could be divided into three stages: (1) reduced renal function without functional evidence of damage to nephrons: (2) more severe reduction in renal function with functional evidence of reversible clannage to nephrons; and (3) the development of a progressive uremia and death with apparently irreversible clanage to nephrons. The first two stages could be produced in dogs by shock resulting from trauma or hemorrhage; the production in the dog of the third stage usually required the clamping of the renal artery for at least four hours.

The kidneys of representative animals from these investigations were studied by us in histological section and by microdissection. It can easily be unclerstood from a consideration of the precarious situation that must have obtained in experiments of the sort clescribed (where the animal is in severe shock or is subjected to such relatively grave experimental procedures as a prolonger clamping of his renal artery and yet must be maintained so as to respond to elaborate functional examinations), that it cannot be hoped that every experiment will approximate the delicate balance that determines the status of the human kiclney in naturally occurring shock. Many of the kidneys, especially those whose renal artery had been clamped four hours, showed gross structural damage far beyond that ever seen in the kidney of shock in man and in other experiments. conversely, not enough structural damage was produced to be comparable with the fatal human renal lesion. The grossly damaged kiclneys, as we shall point out later, were useful, however, as their exaggeration confirmed conclusions derived from the fully successful experiment where both functional and structural lesions reproduced the syndrome seen in the human kidney of shock.

\section{The effect on the kidney of direct ischemia. produced by clamping the renal artery'}

We have found that nephrons from kidneys, the renal artery of which has been clamped four hours or longer, cannot be dissected because the basement membrane of the necrotic tubules, though still visible in sections, is also "necrotic," with the result that the tubules disintegrate completely on maceration and only short remnants and debris can be isolated. It is noteworthy, therefore, that the 


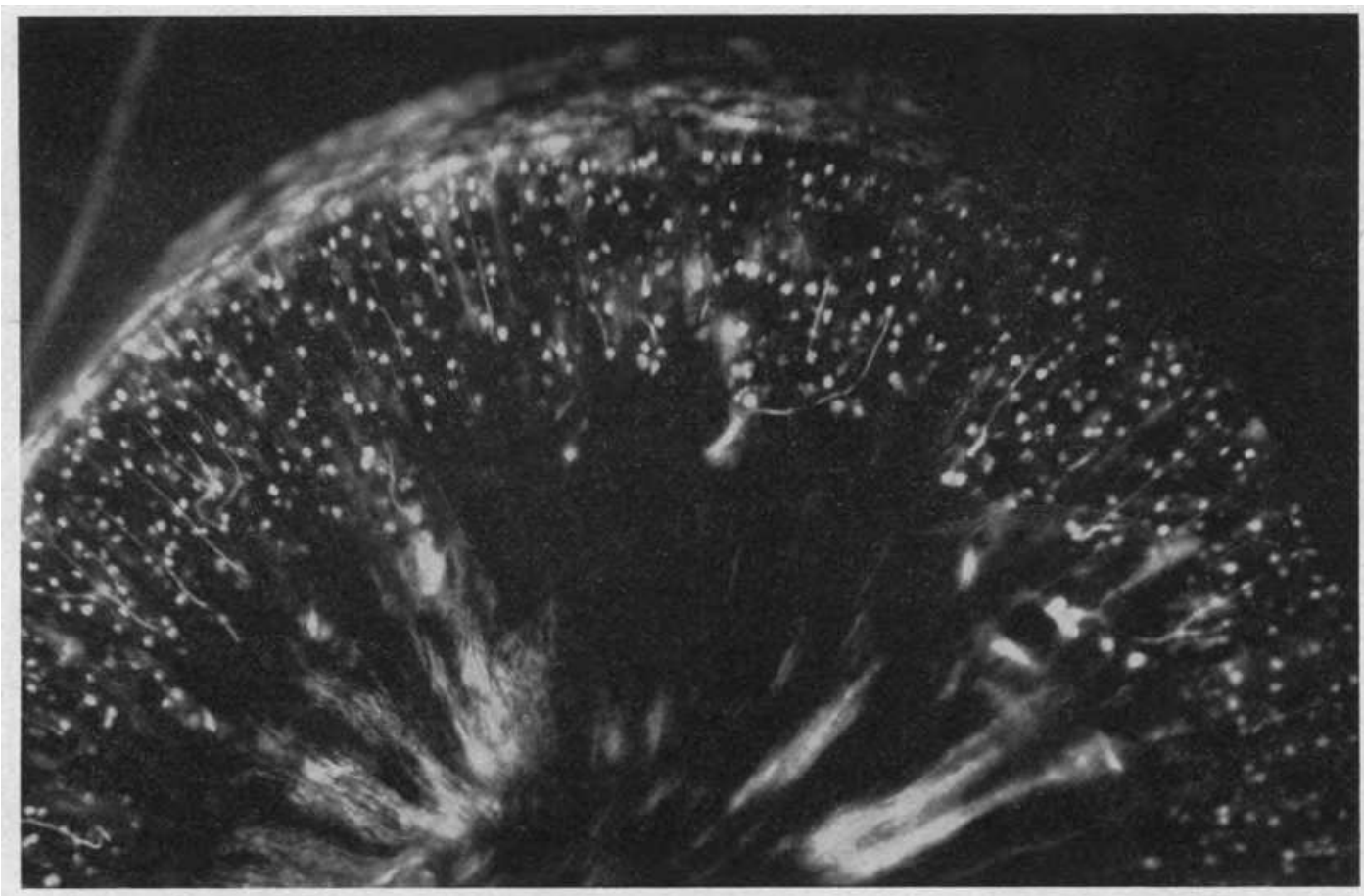

A

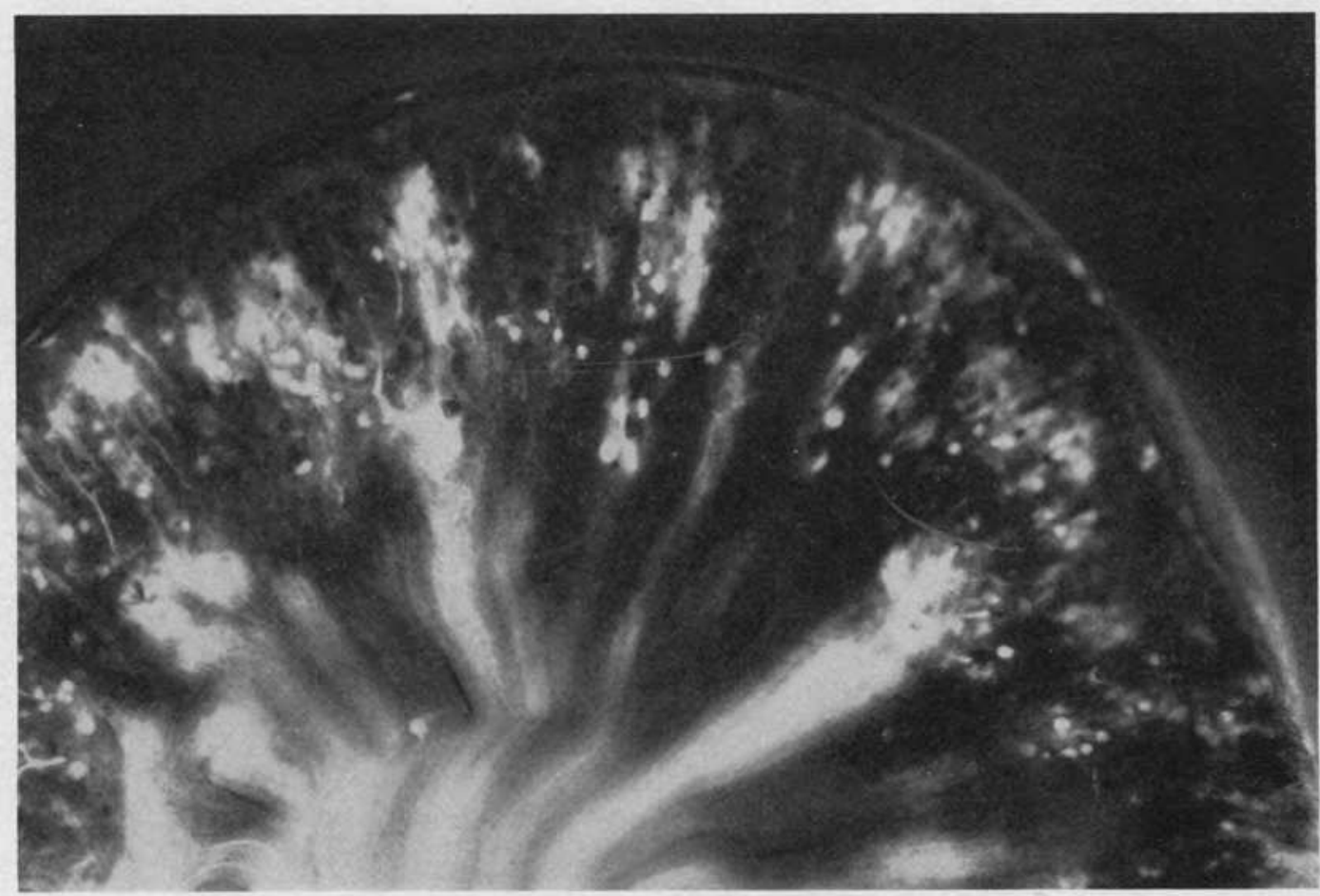

B

Fig. 7. From Schlegel and Moses-Visualization of Kidney Blood Vessels (21)

$A$, the pattern of the normal circulation in the rabbit kidney; B, the patchy ischemia resulting from the action of adrenalin. Reproduced by permission of the authors and publishers. 


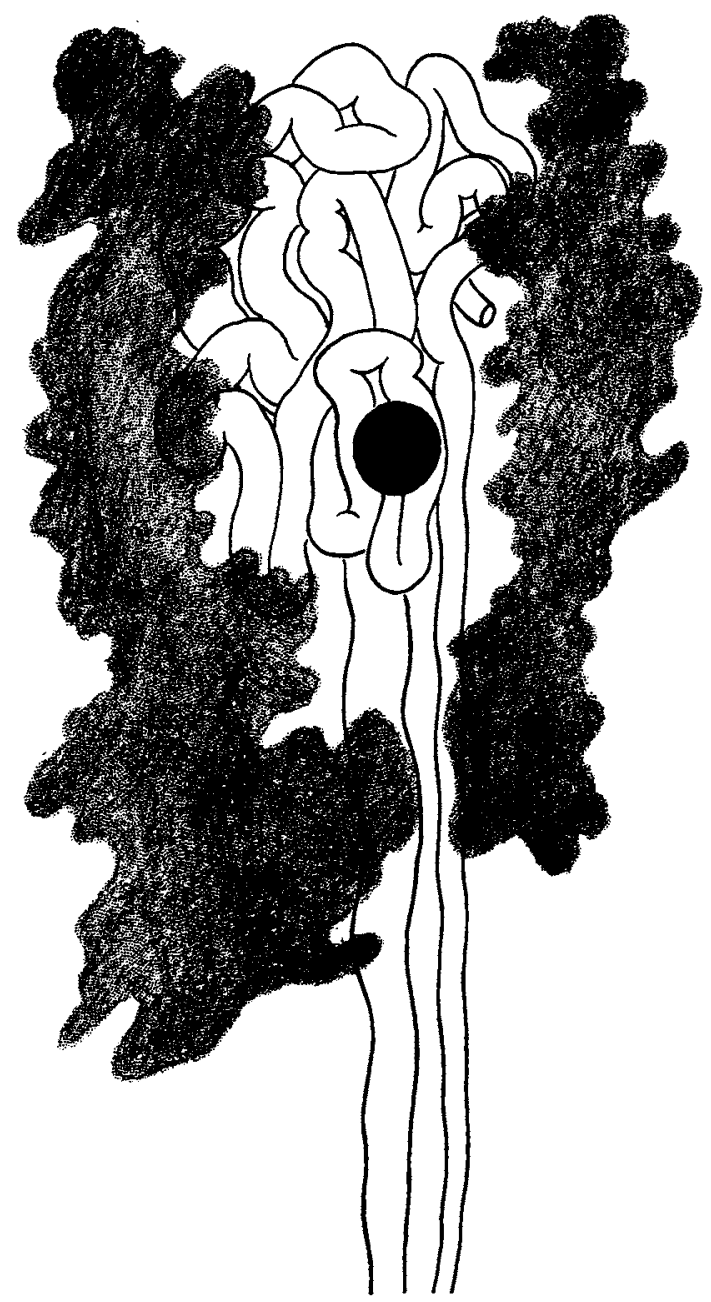

FIG. 8

tubular lesion in these exaggerated instances of experimental ischemic damage show the essential characteristic that was noted in the tubulorhexis of the human case, i.e., dissolution of the basement membrane as well as epithelial necrosis.

Considerably less ischemic damage is shown in the following experiment ( $\operatorname{dog} \mathrm{K} 29$ ). Under nembutal anesthesia the area of the renal vessels was exposed on the left side and two rubber covered serrefines placed on the left renal artery where they remained for 66 minutes. The right kidney remained undisturbed in situ. Seventytwo minutes after the removal of the clamps a $350 \mathrm{cc}$. infusion was given consisting of $50 \mathrm{cc}$. of the animal's red cells laked with $200 \mathrm{cc}$. of distilled water with added salt to isotonicity and $50 \mathrm{cc}$. of the animal's plasma. As one kidney had remained intact in this experiment no functional examinations were made. The animal was killed five days later.

Histological sections of the right kidney showed no lesions; in the left kidney there were irregularly scattered areas of tubular destruction in which proximal convolutions were prominent; approximately one-half of the cortical tissue was thus involved.

Dissections of nephrons showed a peculiarly localized nature of tubular destruction: rarely was the greater part of any portion of a nephron entirely involved. In Plate 28 is shown a complete proximal convolution. Its first portion is completely destroyed, the basement membrane has disintegrated and its epithelium is reduced to debris. The mid-portion then appears remarkably well preserved; even the mitochondrial rodlets, the most sensitive indicator of cellular damage, can be seen and the usual cellular pattern of the unstained nuclei of the renal epithelium is clearly apparent. At the beginning of the medullary segment scattered areas of epithelial necrosis appear which become progressively more extensive until complete epithelial disintegration is observed in its terminal part which narrows to the thin limb of Henle's loop. It should be noted that in the severely damaged terminal portion of the convolution the basement membrane, so clearly seen in the normal middle portion, had disappeared.

A typical lesion in the distal portion of the nephron is shown in Plate 29. The contours of the ascending limb to the left are fairly well preserved but throughout its length irregular collections of cellular debris are seen in the tubule lumen. The distal convolution is filled and irregularly distended with deep black, consolidated haemoglobin casts that were positive to the benzidine test (A) and at (B) a typical disruptive lesion of tubulorhexis is observed. Crystal-like deposits of heme pigment are well shown at this point $\left(\mathrm{B}^{\mathrm{I}}\right)$. It can be observed that from here on the distal and connecting tubule are markedly distended with a solid coagulum of heme pigment yet the tubule wall is intact.

This experiment is of interest in showing that though in histological section the severity of the damage in the proximal convolution masks its essential identity with what is observed in the kid- 
ney of human shock, an examination of the lesion in the isolated nephrons shows not only the same type of limited, disruptive tubular damage but also the same random involvement of scattered stretches of nephron from proximal to distal convolution that is so characteristic of the human lesion.

In experiment $\mathrm{K} 26$ an even closer approximation to the human lesion was obtained. Under nembutal anesthesia the right kidney was removed and a serrefine placed on the left renal artery for 60 minutes. The animal remained in good clinical condition; no functional studies were made and it was killed on the fourth day after the operation.

Sections of the left kidney show none of what is commonly called "frank" necrosis of renal tubules. The epithelial cells in occasional cross sections of a proximal convolution appear desquamated or disarranged but so rare is this appearance among surrounding well-preserved cross sections that a conservative examiner would probably ignore it as some sort of fortuitous artifact. The distal convolutions and collecting tubules contain casts that stain deeply with eosine and which are benzidine positive but negative for free Fe.

Plate 30 shows a complete proximal convolution. The cellular pattern is particularly well shown; at (A) even the mitochondrial rodlets of the epithelium can be made out. At the arrows various degrees of cellular damage, from an irregularity of the mitochondrial pattern to complete necrosis with disruption of the tubule wall, are evident in short isolated segments of the convolution. At (B) are seen short segments of tubule where the basement membrane is destroyed and the necrotic epithelial cells are resolved into dark stained debris. In Plate 31 three distal convolutions join a collecting tubule on the left. In the distal convolutions and collecting tubule (A) can be seen the infrequently mentioned dark staining intercalated cells peculiar to this portion of the nephron. There are many small diverticula on the upper portion of the collecting tubule. There are no casts or damage except in the lowermost distal convolution. At (B) the tubule is disrupted and filled by a black stained plug of heme pigment; at (C) the lumen is packed with similar material but the epithelium is intact.

To summarize these experiments it may be said that under proper conditions of degree and duration, direct ischemia of the kidney following clamping of the renal artery reproduced the essential structural lesions that are observed in the human kidney in shock. In the instances where either the degree or the duration of the ischemia was extreme, the extensive structural damage still showed, in exaggerated form the two characteristics of the renal lesions that are seen in human kidney of shock, namely irregularity of distribution of tubule damage, both within the nephron and throughout the kidney as a whole, and the complete destruction of limited stretches of tubule and basement membrane scattered along the course of a well preserved normal nephron.

\section{The effect on the kidney of ischemia produced by renal vasoconstriction following trauma}

The second group of experiments was analogous in the manner in which renal ischemia was produced to what occurs in the "crush kidney" of man, namely a renal vasoconstriction following as a reflex to trauma of the muscle masses of the limbs. Conversely to the previous experiments where exaggerated effects were more easily produced, in these the difficulty of a successful demonstration lay in the production of a sufficient degree and duration of renal ischemia to produce structural lesions and in the necessity at the same time of maintaining the animal in a physiologically adequate condition that would allow the performance of the functional tests (in particular hippurate extraction) that measured the degree of functional damage and renal blood flow. The details of these experimental procedures and the rationale of the functional determination of renal blood flow are given in extenso in the publication of Phillips, Dole, Hamilton, Emerson, Archibald and Van Slyke (22). The kidneys examined were from experiments similar to those shown in Table IV of that article: unfortunately the kidneys from the identical dogs of this table were not available for structural examination, but the functional findings in those whose structural status is here reported were similar. The rate of hippurate excretion during the course of each experiment will be given as an indication of the degree and duration of renal ischemia that existed in the kidneys. 
1. Minimum functional and structural damage (K33). Under deep nembutal anesthesia, with the customary priming dose and sustaining infusion of $\mathrm{PAH}$, trauma to the legs was begun at minute 92 of the experiment. At 94 the PAH excreted had fallen from $5320 \gamma / \mathrm{min}$. to $709 \gamma$, at minute 119 to $11 \gamma$, and at 131 to $1 \gamma / \mathrm{min}$. The trauma was interruptedly repeated from minutes 134 until 301, and during this period the average of $\mathrm{PAH}$ was $4.7 \gamma / \mathrm{min}$. A transfusion of the dog's own blood at minute 303 was followed by a rise of excretion to $33 \mathrm{\gamma} / \mathrm{min}$. and at minute 397 , to $2890 \mathrm{\gamma} / \mathrm{min}$. On the fourth day, though the animal's clinical condition was good, it was sacrificed. The blood urea $\mathrm{N}$ preceding the experiments was $15 \mathrm{mg}$. per cent. It rose to 24.8 on the second day and was 14.5 before death. Judging from the functional examination it might be said that although there was a period of extreme renal ischemia for 200 minutes the functional damage was reversible and not severe.

Histological section of the kidney showed only slight evidence of damage, much less than that seen in even the least damaged kidneys of the previous experiments where the renal artery was clamped. Benzidine positive casts, however, were frequent in the distal convolutions.

Dissections of nephrons showed the majority to be well preserved; many, however, showed the lesions to be described.

The lesions in the damaged nephrons were entirely similar in distribution throughout the nephron and in their nature to those seen in the former experiments of clamping the renal artery and to those that we have described in the human cases. Plate 32 shows a typical complete proximal convolution. The normal epithelial pattern of clear nuclei in a dark background of cellular granular protoplasm exists throughout the greater part of the convolution. At (A) the tubule is swollen, the epithelium entirely necrotic and the basement membrane disrupted. The detail of these lesions is shown in the inserts of Plate 33. The arrows indicate where lesser degrees of similar damage may be seen. The terminal segment contains, as it normally does in the $d o g,{ }^{4}$ a con-

4 The presence of fat in the terminal portion of the proximal convolution of the normal dog nephron is wellknown to anatomists (23) but less so to some experimentalists. As a result there quite regularly appears in siderable amount of fat which stains so black that cellular detail is obscured. In Plate 33 is shown a typical ascending limb and distal convolution. The first portion of the former contains isolated casts of black stained heme pigment; its upper portion and all of the distal convolution is distended and filled with a solid continuous heme stained coagulum. The cellular detail is thereby completely obscured, but the damage to the basement membrane of the distal convolution is evident from the adhesions which surround the tubule wall.

2. Severe functional and structural damage (K32.) The experimental procedure was essentially similar to the preceding experiment. At 21 minutes the priming dose of $\mathrm{PAH}$ was begun. At 49 minutes PAH excreted was $3100 \gamma / \mathrm{min}$. Trauma was begun at 70 minutes; at 80 minutes excretion had dropped to $1660 \gamma / \mathrm{min}$. The trauma was repeated at intervals and at $159 \mathrm{~min}-$ utes PAH excretion was $3.0 \gamma / \mathrm{min}$. and fell progressively to $1.5 \gamma / \mathrm{min}$. At 305 minutes $1000 \mathrm{cc}$. of a whole blood transfusion was begun; to the blood were added $35 \mathrm{cc}$. of laked blood cells. The animal responded well. Before the experiment the blood urea nitrogen was $10.9 \mathrm{mg}$. per cent. On the fourth day it was 25.4 and on the seventh day, 79.4. The animal, having convulsions, was sacrificed.

Histological sections of both kidneys showed moderate but definite damage to tubules. An occasional cross section of a proximal convolution could be found with definite necrosis of epithelial cells. There were many heme pigment casts, positive to benzidine but negative to the Berlin Blue reaction, in distal convolutions and definite epithelial necrosis in "straight tubules" which might be either ascending limbs or medullary segments of proximal convolution.

On dissection a relatively large number of nephrons, definitely the majority, showed varying degrees of damage. In Plate 34 is seen a com-

the literature correlation of various procedures with the experimenter's discovery of this normal anatomical fact. In the last two years the senior author can recall three sich instances; two in which dietary factors and one in which a neurological lesion were implicated. There will doubtless be more, so that the general reader may care to keep this bit of elementary histology in mind for future critical use. 
plete proximal convolution. The first two thirds are fairly well preserved though stretches are seen where the cell pattern is disturbed. Beginning in the terminal medullary segment (A) there is frank necrosis of epithelial cells and disintegration of the basement membrane with resulting peritubular adhesions. From this point on to the end of the convolution is seen an extensive and complete necrosis of the tubule. The detail of these lesions is shown in Plate 35 at a higher magnification.

Ascending limbs and distal convolutions also frequently showed typical tubulorhextic lesions (Plate 36). The cellular pattern of the lower portion of the ascending limb is visible; its upper third contains black stained heme pigment casts and it passes into a distal convolution that also is distended and plugged with a similar material. The wall of the distal convolution is disrupted and there are the usual peritubular adhesions.

To summarize: in these experiments where renal ischemia followed upon shock produced by trauma to muscle, lesions of the nephrons were produced similar in their nature and distribution to those that were found in the human "crush kidney."

One point stands out more clearly in the experiments than in the human examples of the renal lesion, for in them it is plainly evident that the degree of damage in the kidney of shock is largely a function of the number of nephrons affected by the ischemic insult and not of the severity of the lesion in the nephrons per se. The few nephrons of the first experiment where renal function was not greatly reduced were damaged as severely and in the same manner as the many damaged nephrons of the third experiment in which renal failure resulted. This is in striking contrast with the situation in the kidney of widespread nephrotoxic tubular damage where, since all nephrons are universally affected by the toxic insult, it is the degree of nephron damage that determines the total functional result.

Another apparently minor point, but one to which we shall later refer in our discussion, is established by the experimental procedure. This is the remarkable cytological normality of the epithelial cells of the tubule in regions other than where ischemic disruptive tubulorhexis has occurred. The inmediate fixation of the tissues that is possible in the experiment allows the observation of such detail as the intactness of the mitochondrial rodlets in the cells throughout the nephron immediately up to the point of disruption (Insert, Plate 28). There is therefore no evidence of any toxic damage in the experimental lesion produced by the identical mechanisms that are the cause of the "crush" kidney in man.

\section{The heme pigment metabolism in the kidney of shock}

We have previously described the occurrence of heme pigments only as they appear in casts in the distal segments of the nephron. These deposits lie in the lumen of the tubules and therefore outside the kidney tissue and from their histochemical reactions, i.e., positive benzidine test and negative Berlin Blue reaction, have undergone no apparent alteration in their chemical structure since they give the same reactions to these tests as does haemoglobin. As it is well known that heme pigments filtering through the glomerular membrane under a variety of circumstances are absorbed by the epithelial cells and are there broken down to simpler degradation products, it would seem warranted to consider these processes as a special case of the metabolic mechanism by which the kidney handles proteins (9). Recent studies of these activities in the case of blood pigments may be found in the work of Rather (18) and Lippman, Ureen and Oliver (24).

In our earlier discussion concerning the finding of heme pigments in the casts of the human "crush" kidney the comment was made that it seems singular that so little mention is made in the current literature of these processes in the proximal convolution. The presence of the pigment in the distal portion of the nephron is proof that the cells of the proximal portions must have been in contact with these materials, so that the absence of the usual absorptive activity in the proximal convolution requires an explanation. Considerable light is thrown on this anomalous situation by an examination of the material from the experiments of Van Slyke and his co-workers and comparison of these findings with those of the human material.

The full picture of the renal metabolism of the heme pigments is seen in those experiments where the more natural and moderate mechanisms of 
reflex vasoconstriction following trauma were the cause of ischemia and nephron damage. In these experiments the ischemia, though extreme and of more than one hour duration, was not absolute as it was in those in which the renal artery was clamped. Three potential sources from which heme pigments may enter the tubule lumen are evident in the experiments; namely, as a result of the glomerular filtration of haemoglobin free in the circulating blood, from the transfusion of whole blood even when not followed by any perceptible clinical reaction and from the entrance of myoglobin and possibly also haemoglobin as a result of muscle trauma. In experiment $\mathrm{K} 32$ the urine was noted as "blood tinged" 28 minutes after trauma was begun and 207 minutes before a transfusion was given. A fourth source may also be mentioned in passing though there is no direct evidence of it in the experiments, namely the passage of red cells through glomeruli made more permeable by ischemic damage followed by haemolysis of them in the tubule lumen. At least there seems no other source of the few benzidine casts found in experiment K26 where the renal artery was clamped but no transfusion or trauma to muscles occurred.

In histological sections of all of the trauma experiments (K31-33 and K35) whether laked blood or an apparently reactionless whole blood transfusion had been given, the epithelial cells of those parts of the proximal convolutions which had escaped damage, a considerable portion as judged by the intact mitochondrial pattern seen in Plate 32, were filled with fine benzidine positive droplets. There was also, as is always observed in normal renal cells absorbing heme pigments, a light diffuse tinging of the protoplasm by the benzidine reaction. In the occasional cross section that showed damage in the form of disruption or desquamation of epithelial cells only a very light diffuse staining was evident and few if any droplets. In the lumen of the proximal convolutions there was also seen granular detritus or debris that was benzidine positive, but in straight tubules in the outer stripe of the medulla, presumably ascending limbs, and in the periglomerular loops of the distal convolution, deep brownish yellow stained, well-consolidated casts were present. There were also many benzidine positively reacting casts in collecting tubules.
As all these animals had lived several days, the degradation of the heme pigments could be followed. The Berlin Blue reaction was strongly positive in all the cells of the proximal convolution that the benzidine stain had showed to contain heme pigment. The free $\mathrm{Fe}$ existed either as discrete granules or as a bluish tinge of dropletlike structures. Within the lumen of the proximal convolution the debris was faintly positive for free $\mathrm{Fe}$, but the massive well-consolidated casts in the ascending limbs, distal convolutions and collecting tubules were negative.

In those experiments where an absolute ischemia for three or more hours had followed a clamping of the renal artery no evidence of heme pigment absorption or degradation was found in the severely damaged cells of the proximal convolution even when copious infusions of laked cells or whole blood had followed the unclamping of the artery. The necrotic cells of proximal convolutions were occasionally tinged a faintly diffuse but perceptible yellow, indicating that heme pigment in solution had seeped through the dead tubule wall, and in such kidneys there were many heme pigment casts in ascending limbs, distal convolutions and collecting tubules that were strongly positive to benzidine. It seems therefore reasonable to conclude that the extreme structural tubular damage, including necrosis, of great stretches of proximal convolution had abolished the functional activity of pigment absorption. As a consequence of this absence of absorption there resulted a negative reaction of the proximal convolutions to the Berlin Blue test which is the indication of heme pigment degradation.

In certain experiments where transfusions or haemoglobin infusion followed one hour after total ischemia from a clamping of the renal artery, lengths of proximal convolution remained undamaged at least in the structural sense and yet in these structurally intact cells there were observed only traces of a diffuse positive reaction with benzidine and no well formed droplets. In the lumen of the lower reaches of the nephron the consolidated heme casts were strongly positive. As might be expected, no free Fe resulting from intracellular pigment degradation was found in the cells or lumen of any region of the nephron. The cellular damage responsible for this failure of cellular activity was invisible to the methods of 
examination that were available and in this restricted sense the lesion may be said to be "functional" (25).

To summarize: in all the experiments, whether the ischemia was the result of clamping the renal artery or of reflex renal vascular spasm following muscle trauma, heme pigment flowed through proximal convolutions and was consolidated into casts in the distal nephron. Such pigment at no time was intracellular and therefore was not broken down to show free $\mathrm{Fe}$. In the experiments where the ischemia was the result of the more natural and moderate mechanism of reflex vascular spasm and where large stretches of proximal convolution remained undamaged, absorption of heme pigment in droplet form and subsequent degradation to a Fe free form was observed. In the more drastic and total ischemia following clamping of the renal artery where proximal convolutions were extensively damaged no absorption of heme pigment and consequently no degradation products occurred. Occasionally proximal convolutions, which by present methods of examination appeared structurally intact, contained no pigment.

The experiments therefore supply a morphological demonstration of variations in the damage that may occur to one of the functions of the nephron, namely protein absorption. The phenomena of heme pigment metabolism differ strikingly in their manifestations even in the relatively uniform group of experimental animals. It is therefore not surprising to note even more remarkable variation among the examples of the human shock kidneys, where conditions of origin and subsequent clinical course were extremely varied. The one manifestation previously noted by all writers was consistently found in our material; that is, the presence of heme pigment positive to benzidine and negative to Berlin Blue test in the casts of the distal convolution and collecting tubules; it was the rare example where either the benzidine or Berlin Blue reaction showed that any of this material had been absorbed in the preceding proximal convolutions. In only two examples was the typical and complete picture of heme pigment metabolism present. One of these was a case of transfusion reaction following shock after prostatectomy and the other a case of blackwater fever. In both instances not only were the benzidine positive casts present in the distal nephron but droplets positive to both benzidine and the Berlin Blue reaction were seen in the proximal convolutions in histological section and dissected nephrons (Plate 12).

One case of blackwater fever deserves further comment. In histological section the cells of its proximal convolutions were distended with large droplets that stained heavily with iron haemotoxylin and benzidine. That this was an exaggerated example of the condition was clear from the appearance of the kidneys: the formalin-fixed tissues of the gross specimen were almost black and in sections not only was intracellular and intratubular pigment present, but even the interstitial tissue as well was impregnated with "formalin precipitate" and benzidine positive haemoglobin. Though postmortem diffusion may be in part the source of this impregnation, excessive quantities of the pigment from violently acute intravascular haemolysis might well be expected to appear in the interstitial fluid of a kidney, the nephrons of which are damaged to the point of physical rupture. We believe therefore that this case shows in visible pigmented form, that leakage of proteincontaining fluid from the lumens of disrupted tubules the significance of which in the production of alterations in renal function will receive further attention in our discussion.

Our experience derived from the study of human lesions viewed in the light of the experimental findings therefore suggests that though the typical phenomena of heme pigment metabolism may occur in the shock kidney of man its manifestations are usually modified and obscured. If enough pigment reaches the tubular lumen of the proximal convolutions over a sufficient period of time and if the cells of the proximal convolutions have not been too greatly damaged, as they were for example in the experimental kidneys where the renal artery was clamped, then in addition to the distal pigment casts there may be found the evidence of tubular absorption of heme pigment and protein degradation in the proximal convolution. Renal ischemia, which we can now believe to be the essential cause of the disruptive tubular lesion in man, can therefore, as in the experimental animals, quite adequately account for the functional failure of absorption in the proximal convolutions as well as for the associated structural lesions. 
AN EXPERIMENTAL EXAMINATION OF THE RELATION OF TOXIC AND ISCHEMIC DAMAGE IN THE KIDNEY OF ACUTE RENAL FAILLRE

The structural characteristic of the kidney in fatal Acute Renal Failure that clifferentiates this condition from other nephropathies is found not in the location of tubular lesions but in their nature. We have shown that the Dumn-GillespieNiven lesion, to which for convenience we have given the name of tubulorhexis, occurs at random in all parts of the nephron, that it consists of a peculiar form of disruption of the entire tubule wall and that such randomness and disruptive damage can be explained as at result of ischemia. These conclusions, drawn from a study of the spontaneous lesions seen in man, are confirmed by the experimental reproduction by Van Slyke and his co-workers of the lesion in dogs by renal ischemia resulting from various procedures, including traumatic shock, an admittedly important source of the renal disturbance in man.

It has also been shown that another type of tubular damage is frequently found in the human kidney of Acute Renal Failure, namely various "degenerations" and necrosis of the epithelium of the proximal convolutions; these lesions have all the structural appearances of the action of a toxic agent.

This confusing combination in a single kidney of tubular lesions of such different nature and origin constitutes one of the major difficulties in current concepts since it lies at the very origin of the renal disturbance. The confusion may be readily cleared if one accepts what seems to the pathologist the obvious solution; namely that in certain situations there are two sorts of renal damage in the final picture, each due to its own mechanism. Renal ischemia, a result of circulatory imbalance or "shock," might well complicate a severe intoxication which has produced its usual nephrotoxic effect on the renal tubule and so produce in addition the random tubulorhextic lesions that characterize the full-blown kidney damage that is seen in certain examples of fatal Acute Renal Failure. The drinking of an anti-freeze compound, or any other renal poison, would therefore bear the same relation to the final renal status as does a traumatic crush accident, the convulsions of eclampsia or a transfusion mishap. By this concept the difference between Acute
Renal Failure following poisoning and that resulting from the effects of traumatic injury and shock would lie in the fact that in the case of poisoning the effects of two renal insults are present rather than one.

This conclusion arising from the structural aspect of the lesion is in fact supported by the results of the clinical-functional study of the disturbances observed in certain chemical intoxications that conclude with the suggestion by the investigator that there may be some combination of the effects of specific nephropathic action and ischemia. Sirota (26), for example, in his study of carbon tetrachloride intoxication and Marshall and Hoffman (27) so interpret the results of their studies by clearance methods.

If it is difficult or at present impossible to distinguish such pathogenetic minutiae in the complexity of the clinical case, it should be possible to demonstrate these distinctions under experimental conditions. Certain nephrotoxic substances produce, under proper conditions, exquisitely localized tubular damage. It was shown in 1912 by Susuki (14) that uranium caused tulbular necrosis in the middle third of the proximal convolution and not elsewhere, and this finding, a difficult if not precarious conclusion on the evidence available in histological sections, was confirmed by microdissection of complete nephrons by the senior author in 1915 (28).

Any morpholugist who has been practically concerned with the study in histological sections of considerable numbers of animals poisoned with uranium or any other heavy metal has doubtless had the experience of seeing tubular lesions which. though obviously predominant in the proximal convolution, seem at times to involve other portions of the nephron; the ascending limb of Henle's loop, for example. Appreciating the difficulty or impossibility of exact orientation in sections, such apparent discrepancies are usually passed without comment, but do leave a certain scepticism in the mind of the observer as to the exactness of Susuki's description. It will now be shown that under some circumstances there is real cause for doubt; other parts of the nephron besides the proximal convolution can be seen to be damaged in dissected specimens. Moreover it will be shown by visual demonstration that in these kidneys there existed at some time prior to the sacrifice of the 
animal a patchy ischemia and that in dissected nephrons two sorts of lesions are found; first, the distinctive nephrotoxic damage in the proximal convolutions of all nephrons and secondly, random tubulorhextic disruption in various and indiscriminate parts of some.

In the following experiments a functional disturbance in the renal vascular bed was produced in addition to the nephrotoxic tubular lesion by means of large doses of toxic agents. The effect on the vascular system in the kidney was directly observed at varying periods of the intoxication by the method of Schlegel (2) that we have previously mentioned.

A series of 65 animals were used in the visualization of the renal circulation, 20 as controls to establish the constancy of the normal pattern and 45 that were given the following well-known nephrotoxic poisons: uranyl nitrate, potassium bichromate, corrosive sublimate, carbon tetrachloride, sodium potassium tartrate and di-ethylene glycol.

The procedure of vascular visualization was the same in all experiments. The animal was placed under Dial anesthesia, the abdomen opened and then closed by clamps so that the kidneys could be removed in a matter of a few seconds. After a one-half hour interval, $1.0 \mathrm{cc}$. of 4 per cent thioflavin $\mathrm{S}$ solution per kilo was injected into the ear vein in a three second period, one renal artery clamped and the kidney removed at a measured time interval of six to eight seconds from the beginning of the injection. Free hand slices, both sagittal and longitudinal, were immersed in glycerin and observed with a binocular microscope under incident Wood's light at a magnification of 8 to $12 \times$.

The pattern seen in the normal animal is remarkably constant (Plate 37A). All the glomeruli throughout the cortex are evenly and brilliantly illuminated and no dark glomeruli that did not receive the dye by way of the circulating blood are evident. The cortical background of tubules is faintly illumined by the natural fluorescence of the renal tissue and by the small amount of dye which has diffused out of the vessels. The intensity of the relative contrast between vessels and glomeruli and background tissue therefore depends on avoiding this diffusion; hence the need of speed in removing the kidney as soon as the blood has fully perfused the organ. It may be recalled that the circulation time in the rabbit is of the order of 7.0 seconds so that three seconds is ample time to accomplish the optimal visual effect.

On the faintly fluorescent background of the cortex are seen the brilliantly illuminated sections of the arterial tree as the chance cut of the razor has exposed them; large branches of the renal artery, "arcuate" arteries, and interlobulars with, in the fortunate section, appended delicate afferent vessels and glomeruli and, less clearly, the irregular plexus of intertubular capillaries and venules. In the medulla a brighter zone of primary tissuefluorescence is present in which lie more brilliant efferent capillaries from the cortex. For a more detailed description of this functional-anatomical presentation of the renal circulation the reader is referred to the studies of Schlegel and Moses (21). ${ }^{5}$

The general appearance of the renal circulation at periods from $3 \frac{1}{2}$ to 70 hours after the administration of the poisons was relatively similar. All showed in varying degree the pattern of an irregular cortical ischemia.

Plate 37B shows the kidney 19 hours after the subcutaneous injection of $400 \mathrm{mg}$. per kilo of sodium potassium tartrate. There is an extreme ischemia with an irregular area to the left where considerable blood and dye have reached the glomeruli; in this area diffusion through the vessel walls is pronounced.

In Plate $38 \mathrm{~A}$ is shown the vascular pattern $4 \frac{1}{2}$ hours after the intravenous injection of $24 \mathrm{mg}$. of corrosive sublimate per kilo. On the greyish, natural fluorescence of the renal cortex only a few scattered, irregularly illuminated glomeruli and cut branches of the smaller renal arteries are seen. The second kidney (38B) into which

5 The pattern of the renal circulation displayed by this technique which depends solely on the functional forces that operate under physiological conditions is strikingly different from that produced by the artificial injection under pressure of various substances that hitherto have been the main source of our visual impressions of the nature of the renal circulation. No matter how carefully selected the injection material and how meticulously regulated the pressure, it is apparent that the distended, tortuously obese appearance of capillaries and glomeruli which forms the subconscious basis of our present concept of the renal circulatory bed is an artifact that is never simulated under living conditions. 
blood was deflected by the clamping of the opposite renal artery and which therefore had received blood for a period three times as long as the first, thus allowing the accumulation of more dye in the vessel wall, shows more and brighter glomeruli and vessels but a comparison with the even pattern of the normal kidney (Plate 37A) which received dye over a lesser period, indicates that there is a persistence of irregular areas of cortical ischemia where no glomeruli are evident.

In Plate 39A, uranyl nitrate, $40 \mathrm{mg}$. intravenously per kilo after 70 hours, an irregular area of dark ischemia is seen in the center of the cortex. Plate 39B shows the kidney 19 hours after an intravenous injection of $30 \mathrm{mg}$. per kilo of potassium bichromate. The ischemia of the cortex is obvious.

Plate $40 \mathrm{~A}$ shows the kidney removed on the second day after two subcutaneous injections of $4.0 \mathrm{cc}$. carbon tetrachloride; in Plate $40 \mathrm{~B}$ the kidney after a 24 hour period in which the animal took ad lib. 5 per cent di-ethylene glycol in his drinking water. In both the irregular pattern of cortical ischemia is evident.

A visualization of the renal vessels at various periods after the administration and during the action of nephrotoxic agents leaves no doubt that over an extended time there is an irregularly distributed disturbance in blood flow through the renal cortex. A comparison of the resulting vascular pattern with that which Schlegel and Moses (21) have demonstrated to follow the injection of adrenalin, a form of renal ischemia concerning whose mechanism of origin we have more information, allows certain speculative interpretations in regard to the situation that obtains in the cortical ischemias following renal poisons.

As Schlegel and Moses have shown, and as might be expected from our pharmacological knowlèdge of its mode of action, the renal ischemia of adrenalin action is abrupt in onset, intense in degree and relatively short in duration. In combination with the apparent irregularity of its action on the terminal branches of the renal arterial bed, these factors produce temporarily patches of almost bloodless cortical tissue in which, at times, can be seen totally ischemic glomeruli. There is also an apparent decrease in blood flow through the medulla. Such is the picture that might be expected to follow a sudden, complete, but evanescent spasm of small arteries, presumably chiefly interlobulars and glomerulus afferents.

Judging from the appearance of the toxic ischemic vascular pattern it would seem that the ischemia, though equally irregular in its distribution, is less absolute; a few shrunken, i.e., not fully irrigated, dimly illuminated glomeruli are scattered throughout the relatively bloodless regions giving an appearance that suggests that a mere trickle of dye-containing blood had been flowing in the involved areas. This impression is strengthened by the appearance of the second kidney, if it is removed and examined after a period of dyecontaining blood flow two to three times as long as that which had occurred in the first kidney: more glomeruli and vessels are seen to be fluorescent, though there still is nothing comparable to the even, bright pattern of the normal animal. Evidently the dye can accumulate in the wall of the vessel with time even with a considerable reduction of blood flow. Another effect that results in a somewhat different picture of toxic cortical ischemia from that seen after the action of adrenalin is possibly due to damage to the vessel wall and an increased diffusion of the dye into the cortical tissue.

The similarities and differences between the two patterns of vascular disturbance show therefore that in the case of severe nephrotoxic poisoning there is a long-continued, irregularly distributed ischemia of the renal cortex, a potential cause, as the Van Slyke-Phillips experiments demonstrated, of irregularly scattered disruptive tubulorhextic lesions. Dissections of the nephrons from the kidneys of the animals of our experiments show that, besides the lesions in proximal convolutions due to the poison, the random disruptive lesions are in fact present.

There was considerable variation in the degree of damage noted in the kidneys from the individual experiments; in some the nephrotoxic lesion limited to the proximal convolution alone was evident and the remainder of the nephron was normal except that the lumens of the ascending limbs and distal convolutions were filled with epithelial debris and casts. The epithelium of these latter segments though compressed showed well preserved nuclei and even a normal mitochondrial pattern. In other animals, however, in which a well developed ischemic pattern had been 
demonstrated in the renal cortex there were found typical tubulorhextic disruptions occurring in all parts of the nephron including the proximal convolutions.

In Plate 41 is seen a proximal convolution from rabbit 60 which had received $0.4 \mathrm{gm}$. of sodium potassium tartrate per kilo subcutaneously and which was killed 19 hours later. The ischemic pattern in this animal is shown in Plate 37B. It will be noted that in the first few coils of the convolution the nuclei may be faintly seen in the heavily stained cytoplasm of the intact epithelium; from this point onward the tubule is swollen and the necrotic epithelial cells have disintegrated into cellular debris. This lesion is that of typical nephrotoxic damage: at $\mathrm{A}$, however, the basement membrane is ruptured and a short segment of tubule shows the tubulorhextic lesion. Similar disruptive lesions are found in the ascending limbs of Henle's loop and in the distal convolution. Plate $42 \mathrm{~A}$ from another animal which received the same dosage and was killed on the second day shows an extreme form of the lesion, with dissolution of the basement membrane throughout and, in the right descending portion of the illustration, disruption.

In Plates $42 \mathrm{~B}$ and $43 \mathrm{~A}$ are shown distal convolutions and ascending limbs of two rabbits which had been given $40 \mathrm{mg}$. of uranyl nitrate per kilo intravenously and killed 70 hours later. The visualized ischemic pattern of the cortex of Plate $42 \mathrm{~B}$ is shown in Plate 39A. The proximal convolutions of both these animals showed extensive nephrotoxic damage with the typical specific localization in their middle and terminal portions. It will be noticed that the disruption of the tubule wall involves only short segments in both examples and that there is no evidence of a diffuse nephrotoxic necrosis in the remaining portions.

Plate $42 \mathrm{C}$ and $\mathrm{D}$ are from a rabbit that was given $15 \mathrm{mg}$. per kilo of corrosive sublimate and killed 18 hours later. The pattern of cortical ischemia is seen in Plate $38 \mathrm{~A}$ and $\mathrm{B}$. The proximal convolutions showed typical nephrotoxic damage in the middle and terminal portions. The short segment of disruptive tubulorhexis at the junction of ascending limb and distal convolution is clearly evident, while in both preparations the normal appearance of the distal convolution indi- cates that there was no general toxic involvement of its epithelium.

The disruptive effect of ischemia occurring in di-ethylene glycol poisoning in rabbits which had taken ad lib. this toxic agent in 5 per cent solution in their drinking water for five and seven days is seen in Plate $43 \mathrm{~B}$ and $\mathrm{C}$ and Plate 42E. Lesions of slight degree and moderate extent are shown in plates $42 \mathrm{E}$ and $43 \mathrm{~B}$; note the normal nuclei in both ascending limb and distal convolution except where the ischemic disruptive lesion has occurred. In Plate $43 \mathrm{C}$ there is extensive and extreme damage with dissolution of the basement membrane and destruction of epithelium throughout two greatly swollen ascending limbs and distal convolutions from another animal which had taken the poison for seven days. Disruption has occurred where the two distals are adherent and there is a large collection of black stained debris at this point. The patchy cortical ischemia of a similar animal is shown in Plate 40B. The nephrons of all these animals showed an extensive nephrotoxic necrosis in the terminal half of the proximal convolution, this portion being greatly swollen; even the typical vacuolated appearance of the necrotic epithelium is evident (Plate 44). There is no disruptive ischemic lesion in this proximal convolution but others showed irregularly scattered segments of tubulorhexis.

In Plate 43D the disruptive lesions associated with the cortical ischemia (Plate 40A) that followed 48 hours after subcutaneous injection of 4 cc. per kilo of carbon tetrachloride are illustrated. The areas of tubular damage are quite small and localized and complete disruption of the tubule wall has not resulted. The proximal convolutions showed a typical nephrotoxic necrosis of the epithelium of their terminal portion.

In summary, the lesions seen in the kidneys of the experimental animals poisoned with various nephrotoxic agents all showed the typical involvement of the proximal convolution that is characteristic of this group of substances. Figure 9 shows camera lucida tracings of nephrons with the localization of nephrotoxic damage from various poisons. ${ }^{\circ}$ In other kidneys in which the

6 It should be emphasized that the production of exact differences in localization of the lesion demands careful experimental control for their demonstration: if too large a dose is given the area of damage tends to spread 

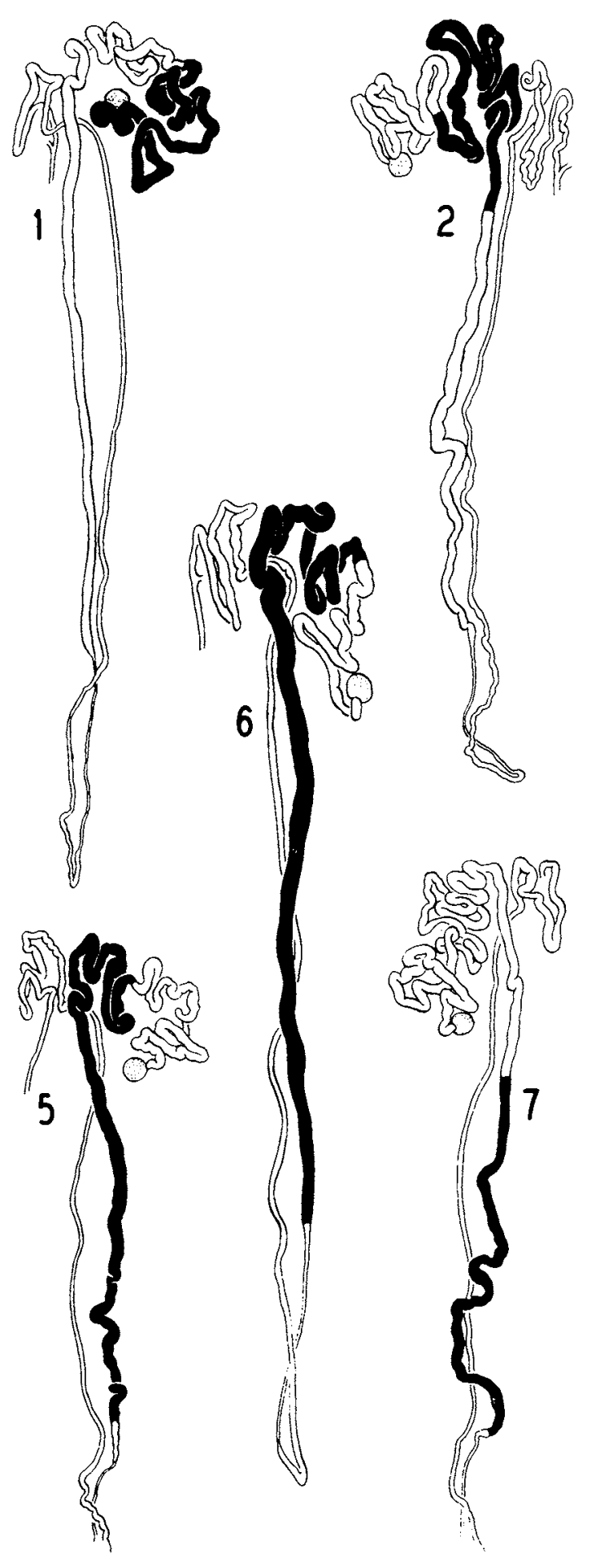

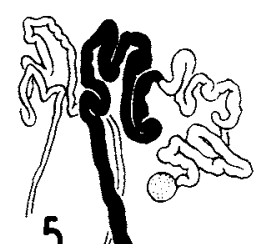

5

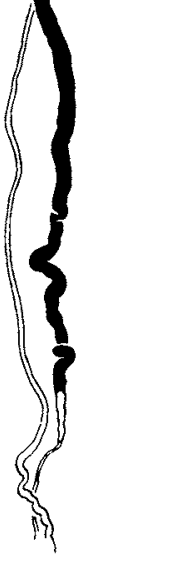

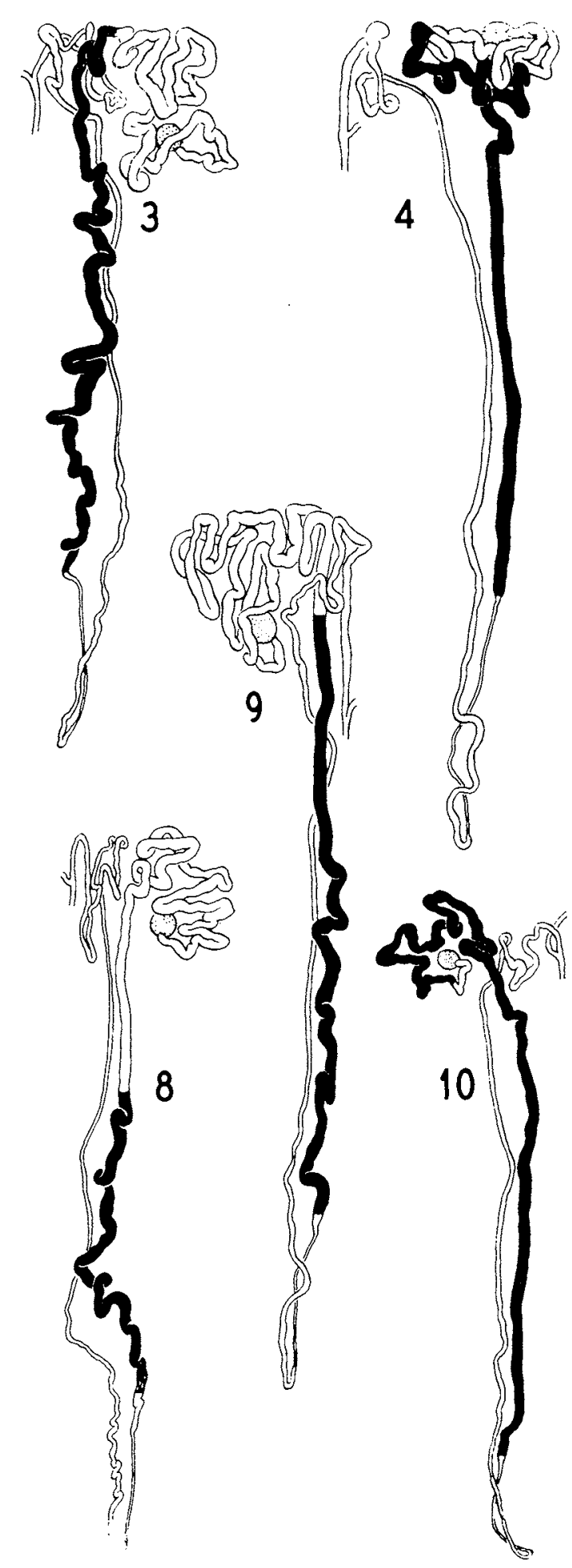

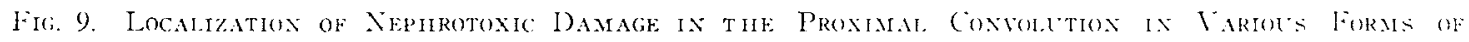
Poisonixe;

1, Potassium bichromate; 2, uranyl nitrate; 3, corrosive sublimate; 4 , solium potassium tartrate: 5, potassium chlorate; 0 , di-ethylene glycol: 7 , carbon tetrachloride; 8 , mushroom poisoning: 9, serine; 10. sulfonamides. The necrotizing effect of moderate doses is shown by black areas; with large doses the area of localization tends to spread and there is the further complication of an ischenuric episcrle with the develoy)ment of discuptive tubulat kesions. Magnification $20 \times$. 
Schlegel technique had demonstrated that there had existed an irregular and patchy cortical ischenia, the disruptive lesions of ischenic damage were found scattered at random throughout the length of the nephrons.

The experimental method, by its opportunities for control in dosage and consequent damage, allowed the demonstration of early forms of these ischemic lesions; Plate 42C, D and $\mathrm{E}$ and Plate $43 \mathrm{~B}$ and $\mathrm{D}$, for example, show short, limited areas of tubule swelling and basement membrane dissolution in stretches of tubule, the epithelial cells of which are generally normal. The similarity of these early lesions to those produced in the Van Slyke-Phillips experiments by purely ischemic insult in which no toxic substance was concerned (Plates 30 and 32) is striking evidence of a different manner of origin of the scattered tubulorhextic lesions from that of the nephrotoxic damage in the proximal convolutions. In our experimental procedure the only factor introduced to cause a disturbance in renal blood flow was the use of large doses of the toxic elements for this was adequate to establish the point of our argument. It seems certain, however, that if the other complicating factors, such as dehydration, vomiting, colitis and even "shock" that accompany fatal human poisoning, had been reproduced in the experiment, then the renal lesions in the animals

along the course of the tubule until in the end the greater part or all of the proximal convolution is affected and the selectivity of the toxic action is lost. There is also, as we have just shown, the complication of renal ischemic damage that accompanies the graver intoxications and this may occur in the proximal convolution as well as elsewhere and so disturb the pattern of the specific lesion. The selective localization of the action of various toxic agents, as observed in the experimental animal, does not therefore, as we have seen in our dissections, commonly occur in human poisoning, but it has nevertheless considerable theoretical importance in regard to the recognition of differences in the seat of functional activities in the proximal convolution (29). The only confirmations by dissection of Susuki's original conclusions, which were drawn from the study of histological sections with which we are familiar, are in the case of uranium (28) and corrosive sublimate (15). During past years a considerable number of other poisons have been studied in this laboratory and these findings are shown in Figure 9. This is not a diagrammatic, i.e., interpretive presentation, but shows camera lucida outline tracings of actual nephrons with the area of nephrotoxic damage indicated by cross hatching. would have departed as far from the classical description of the effects of toxic substances as did those of the human cases of poisoning which we have previously described.

\section{SUMMARY, REITERATION, AND SPECLLATION}

It is clear that what has been revealed by this investigation, both in its examination of the structural status of the human kidneys and by its experimental procedures, does not lead to a confirmation of the currently prevailing concept of a "kidney disease" the "unique" pathological characteristics of which can be described as "lower nephron nephrosis," for the lesions in the kidney are not peculiar to or localized in any portion of the nephron and their diverse character would seem to strain even the meaningless embrace of that most tolerant of terms, "nephrosis." ; Nor are our findings compatible with the present theories of this curious malady, the pathogenesis

7 The term "haemoglobinuric nephrosis" is equally unsatisfactory, for it can hardly be used to apply to the whole group (Acute Renal Failure associated with traumatic and toxic injury) since in many examples hacmoglobin is not concerned at all in the lesion. Even in those in which heme pigments are found either in casts or in the renal cells it is doubtful that they play any part as a "toxic factor" in the production of the characteristic tubular lesions. We have shown that the cells of the tubule are well preserved in the presence of deposits of heme pigments either free or in casts and that it is only coincidentally that lesions and pigments are found together. Extensive experimental evidence indicates that in any moderate amount haemoglobin does not damage the nephron: it is in fact used experimentally as a measure of glomerular permeability and does not sufficiently disturb renal function to interfere with the use of clearance methods. Its appearance in the cells of the proximal convolution in droplet form is a special case of the normal activity of these cells in absorbing protein from the tubule fluid and digesting it (9). In the case of haemoglobin this metabolic activity is indicated by the appearance of free $\mathrm{Fe}$ in the renal cells. It is true that, as in all digestive processes, an over-loading of the mechanisms can produce indigestion with resulting transient ill effects on the digestive organs, in this case the renal cells. A similar indigestion of serum proteins with the development of cellular abnormalities, hyaline droplets, occurs in the nephrotic syndrome of chronic glomerular nephritis, but these tubular alterations are entirely different in character from those which we have been considering. It is also true that too-bountifully a good meal has its ill effects, but one would hesitate to designate this not unpleasant temporary discomfort as a "disease" of the stomach. 
of which fits no functional or anatomical pattern known to the kidney and whose "cause" is sought in toxic factors such as heme pigments or among less determinate entities such as "products of traumatized tissue," "electrolyte imbalance" and even "allergies."

And yet amidst all this elaboration of hypothesis and semantic confusion the syndrome does stand clearly outlined as the most important of the acute disturbances of the urinary system. This place it maintains not only by the frequency of its occurrence and the gravity of its ultimate effects, but more importantly by the remarkably protean aspect of its origin: it would seem that almost any clinical situation-trauma, malarial infection, obstetrical accident, transfusion reaction, postoperative complication or intoxicationmay end with its manifestations of renal failure. There must be, therefore, some pathogenetic unity behind this screen of functional, pathological and clinical diversity.

Dr. Homer Smith solves the nosological puzzle by bringing all Acute Renal Failure together in Chapter XXIV of his recent monograph (1), and it is evident that from the functional viewpoint a most reasonable unity results. But the question may arise if the job has been completely finished, for a morphologist will be disturbed to note that the chapter begins with a consideration of the kidney in sublimate poisoning and ends with the "crush kidney," and from what he knows of the structural changes that occur in these two conditions, the squeezing of any two such "entities" into one pigeon-hole may seem a unification of diversity attained only by considerable Procrustean violence. As he sees quite different structural effects, he will suspect different pathogenetic causes ; the sublimate kidney seems obviously the result of a toxic factor while the functional data stress the importance of some disturbance of renal blood flow in the "crush kidney." Though in the last analysis toxic and ischemic damage may, as interferences with cellular respiration, be similar, at the more immediately accessible level of their mechanisms they seem quite different, a difference indeed that might determine such very practical matters as prognosis and manner of treatment. And so a morphologist justifiably might be doubly perplexed to find not only one but both of his conceptual progeny in this galley of Acute Renal Failure.

Functionally the two examples of the renal lesion are similar, for clearly the kidneys of both sorts have "failed," but this is not enough. Structurally they are different; again apparently the all-too frequent dichotomy of the functional and structural approach. Believing that the best way to solve a dilemma is to avoid it, we suggest, at the hazard of considerable reiteration, that the situation be reviewed from the beginning and, quite naturally, that the start be made from the structural standpoint.

The common structural lesion in all members of the group of Acute Renal Failure is tubular damage, and if a name be required, they may all be said to be examples of a "tubular nephropathy." This is not to assume that the glomeruli are entirely normal; proteinuria and consequent cast formation are clear evidence that the filters are leaking, an abnormality which must be structural in mechanisms which are invisible to present methods of observation. For this practical reason only we suggest the use of the tubular aspect of the renal lesions as the "structural characteristic" of the pathological entity.

When the tubules of the nephrons from a large number of examples of this group of varied clinical origin are examined by a method which adequately displays not only the cytological lesion but the topographical continuity of the nephron, it becomes apparent that there are two quite different sorts of tubular damage.

One sort of tubular damage is the well-known nephrotoxic lesion that results when some poison in the blood stream is distributed evenly and equally to every nephron of the two kidneys. Like many foreign substances, it is filtered through the glomerulus and absorbed by the cells of every proximal convolution. A. considerable intracellular concentration above what exists in the circulating blood is thereby established and the renal cells die. The basement membrane is not visibly affected in this lesion nor are the epithelial cells of the other portions of the nephron; both escape because in regard to the toxic substances they are functionless. The tubule lumen below the site of actural damage may be filled with the products of both the glomerular lesion, that is, coagulated protein in the form of casts, and epithelial destruction. 
The other sort of tubular lesion, first recognized by Dunn, Gillespie and Niven in the "crush kidney" and thought by many to be peculiar to the "lower nephron," is in almost every way the antithesis of the nephrotoxic tubular lesion. Its distribution within the kidneys is entirely random; some nephrons, often in groups, are involved while others are free of it. Within the nephron any portion may be the seat of damage, from the origin of the tubule at the glomerulus to the collecting tubule. One of the most frequent sites is the second half of the proximal convolution which extends into the outer medulla and in histological section is easily confused with the ascending distal limb of Henle's loop.

The lesions themselves differ strikingly in their appearance from what is seen in the nephrotoxic lesion. Rarely is any whole segment of the nephron affected; there may be only a short stretch of tubule or a series of isolated spots that lie distributed over several anatomically and functionally different divisions of the nephron. In these areas the entire tubule wall is affected, including the basement membrane, which may be broken with its remnants still visible or thinned to a barely perceptible sheet or completely disintegrated. The epithelium within it is dead and desquamated so that the result is a disruption of the continuity of the tubule. From this characteristic the term "disruptive" or "tubulorhextic" has been suggested to distinguish it from the nephrotoxic tubular lesion.

If casts are present at the site of the disruptive lesion, and they often are not, they may extrude from the gaping hole in the tubule. In the intertubular connective tissue in the vicinity of the rupture there commonly occurs an inflammatory reaction with leucocytic infiltration and the formation of inflammatory granulation tissue which extends by growth into the lumen of the disrupted tubule; if larger vessels are present "tubulo-venous anastomoses" may form.

If one wishes to speculate as to the origin and cause of a lesion of this sort on the basis of the purely morphological data, a procedure that was necessary in the earlier stages of this investigation, it certainly is quite clear from what is known of the functional and anatomical pattern of the kidney that no such damage can conceivably be the result of a poison reaching the nephrons by the blood stream. This conclusion disposes of a vast amount of speculative hypothetical possibility, such as an origin resulting from "toxic products of tissue damage." A great deal more will have to be said about the part that toxic damage may play in the group of Acute Renal Failure, it is true, but in our immediate and restricted problem, the cause of the disruptive tubular lesion, we can be reasonably satisfied that this element is eliminated.

Another considerable simplification in the decision as to possible causal factors results from the observation of casts, pigment and debris deposits in the continuity of the dissected nephrons for these objects have a much greater extent and complexity than is suggested by their appearance in histological sections. It is readily seen in the dissected nephrons, for example, that extensive deposits of extraordinary length of heme pigment and thick casts, either containing or free of heme pigment, may distend ascending limbs, distal convolutions and connecting tubules and yet a completely intact tubular wall persist in which both the renal epithelial cells and basement membrane, though thinned perhaps by tension, are not otherwise damaged. Moreover casts and pigment deposits are extremely uncommon in the proximal convolution where disruption is frequent. In some instances it is true that casts or pigment deposits coincide with tubular disruption, but the more frequent negative evidence precludes any possibility of a causal relation.

There remains as a causal factor to be considered, the phenomenon of reduced renal blood flow which, as Dr. Smith's chapter emphasizes, accompanies so many of the renal disturbances of this group. This factor would be equally incredible if the current concept of a lesion unique in its localization to the lower nephron were true, but a morphologist would be quick to seize upon the factor of renal ischemia as a likely cause of scattered and random lesions in the kidney for he has seen many such examples of cause and effect in other tissues and organs. Moreover this conclusion from his general experience is made more certain by the visualization of the mechanisms and patterns of renal ischemia by Schlegel and Moses whose technique allows an objective and exact morphological view of the exact status of the renal 
blood flow at any given moment and under any particular condition.

One of the most important contributions of this technique is the clemonstration of the remarkable patchiness of renal ischemia; in the experimental conditions which we have examined there is rarely an over-all, even reduction of flow through the vascular bed; some areas are bloodless, others appear congested. In viewing this total pattern one might wonder if there had been much if any reduction in total renal blood flow but rather if there had not occurred only an irregular distribution of blood flow within the cortical tissue. ${ }^{*}$ The implications of this possibility are of considerable importance both to the morphologist and the functionalist. To the former it explains the peculiar random distribution of the lesions he sees in the nephrons and to the latter it may bring further complications in his interpretation of the significance of "clearances" in the pathological kidney for it would appear that some nephrons or even parts of nephrons can be bloodless while others are irrigated with an excess and yet the total blood flow may be approximately normal. In uranium poisoning. for example, clearance methods indicate a normal renal blood flow (1), yet Plate 39A shows a definite, irregular cortical ischemia.

All this preliminary spade work of a priori evidence and speculation as to the part that might be played by renal ischenia in the production of disruptive tubular lesions may well seem to have been a labour of supererogation when we come at

8 In the cortical ischemia following the administration of toxic substances we have never seen a pattern suggesting a deflection or shunt of blood from an anemic cortex to the medulla. In this regard, i.c., the production of the Truetta phenomenon, it might be said in passing that the normal anatomical configuration of the circulatory bed in the kidney is such that any drop in the pressure head in the larger distributing arteries (interlobar or arcuate) will automatically result in a cessation of flow at the periphery of the system, i.c., the terminal branches of the interlobular arteries and so produce a zone of sub-capsular ischemia which, as the pressure head further falls, will spread downwards toward the medulla.

The medulla may thus continue to receive blood from the efferents of the juxta-medullary glomeruli at a time when the cortex is largely ischemic. Such a maldistribution of blood is not a shunt, but only the final stage in the persistance of the failing circulation. last to the actuality of its experimental production by Van Slyke, Phillips and their co-workers. In anatonical material from delicately balanced experiments where the operative margin letween too much and too little must have been peculiarly precarious, our dissections have shown that they produced the typical disruptive lesions both by direct ischemia following clamping the renal artery of dogs and by that which follows reflex renal vasomotor constriction associated with muscular trauma. This demonstration, with the extensive functional evidence which they have produced, would seem to settle the question as to what is the cause of the disruptive tubular lesion in the kiclney of shock. Besides this, the chief contribution of their experiments, there are certain subsidiary eviclences of considerable value.

For example, many attempts have been made to reproduce the "crush kidney" by clamping the renal artery with dubious success. The Van Slyke-Phillips kidneys show clearly in the large series of their experiments every degree of renal damage from what might be called an infarction of the kiclney to the typical, scattered lesions of disruptive tuluular damage in isolated nephrons. Exactly the right degree, both in duration and extent, of renal ischemia is required to produce the distinctive, disruptive lesions of the shock kidney. Moreover earlier investigators were discouraged in their attempts by finding involvement of the proximal convolution when the key lesion they were seeking was presumably confined to the "lower nephron." They could not reproduce the "unique" lesion which does not and, from the structural and functional pattern of the kidney, could not exist.

Another finding of considerable importance in the experimental material was the observation that in tubules containing well developed disruptive lesions as a result of the renal ischemia that followed extensive traumatization of muscle and where heme pigments were excreted into the tubule lumen, absorbed by the epithelium of the proxinal convolution and also deposited unchanged in cast form in the distal convolution, there was no structural evidence of toxic injury to the renal cells, even to those which were contiguous to the points of tubular disruption.

The most sensitive morphological criterion of toxic damage to the renal epithelium requires im- 
mediate and perfect fixation of the tissues and so it cannot be applied to human autopsy material. This evidence of a toxic lesion consists in a dissolution of the mitochondrial rodlets of renal cells, yet in the experimental material these structures were found well preserved throughout proximal convoluted tubules that contained extensive disruptive lesions of ischemia. To the morphologist this seems proof that neither products of the muscle injury nor the heme pigments could have had even a minimal toxic effect on the tubules of these experimental "crush" kidneys.

Though such refined cytological evidence is unavailable in human kidneys from autopsy, the general tenor of the argument would seem to indicate with reasonable certitude the pathogenesis of one member of the group of Acute Renal Failure, namely the "crush kidney" of traumatic injury: the structural lesion is characterized by the disruptive tubular type of damage with no nephrotoxic tubular damage and renal ischemia concomitant with or a sequel to the syndrome of circulatory collapse or general shock is the reason for its occurrence. ${ }^{9}$

To return now to Dr. Smith's category of Acute Renal Failure, it is found that the "crush kidney" comes at the end of a series, the first of which is the renal disturbance produced by uranium poisoning; between the two are distributed functional disturbances that result from the most varied of clinical conditions. Regarded purely on the basis of their structural status no more apposite serial arrangement of the members of the group could be made by the morphologist, for the uranium kidney in its simpler form seens far removed from the "crush kidney," its tubular lesions being by classic description of the nephrotoxic sort with no evidence of involvement of anything but the proximal convolutions and with no disruptive tubular damage.

When two structural lesions are antithetical it might be contended that they can hardly be compatible members of the same pathogenic group and that at last the much desired correlation of our structural-functional data has broken down.

\footnotetext{
${ }^{9}$ In the crush syndrome of man it is of course conceivable that the vasomotor spasm which is the "cause" of the ischemia might be the result of a poison acting on the arteries and arterioles. The "cause" of the epithelial lesion under these hypothetical conditions would remain, however, the ischemia.
}

But on considered thought and in the light of what we have seen in the sublimate kidney of man, the discrepancy is not so real as would appear and it arises only because two very different circumstances of renal insult have been compared, namely one that arises spontaneously under stress in man and another that is produced experimentally in the laboratory animal under carefully controlled conditions.

When a man is poisoned in the course of natural circumstance, by corrosive sublimate for example, in a dosage of uncontrolled amount and passes through periods of excitement and depression, of vomiting and dehydration, of violent enteritis and exhausting diarrhea that end in a state of circulatory collapse, what has occurred to him as a living organism is very different from what has happened in a rabbit which has been given moderate intravenous injection of the same substance and which passes quietly and ostensibly without clinical symptoms or disturbances into renal failure. It is therefore not surprising that the lesions in the human kidneys are not examples of simple nephrotoxic damage, but that there are also present, as evidences of circulatory disturbances, the disruptive tubular lesions of ischemia. We have shown that this is also true in the kidneys of rabbits that have received large doses of nephrotoxic poisons and have demonstrated by visualization of the renal circulation that during the course of these severe intoxications periods of renal ischemia occur which might reasonably account for the mixed nature of the tubular lesions.

To put the varied possibilities in much oversimplified case form, an individual may therefore suffer Acute Renal Failure under the following circumstances :

1. Crushing injury-shock-renal ischemiarandom disruptive tubular damage-anuria-uremia-death.

2. Anti-freeze or sublimate intoxication-diffuse nephrotoxic proximal damage-dehydration, $\mathrm{K}$ poisoning, enteritis, diarrhea-circulatory collapse-renal ischemia-random disruptive tubular lesions-anuria-uremia-death.

3. Moderate poisoning-diffuse nephrotoxic proximal damage - oliguria or temporary anuriaregeneration-recovery.

This last group is obviously a theoretical instance for the pathologist for even in the classical 
and "purest" example of nephrotoxic damage, the sublimate kidney, all fatal cases showed the effects of ischemia by the presence of disruptive tubular damage. It is one, however, which he can accept by analogy from his experimental experience and that of his clinical confreres.

By this concept unity of pathogenesis is obtained in both the structural and functional aspects of a renal syndrome that seems at first glance to be a bewildering clinical congeries of placental separations, transfusion accidents, traumatic or surgical shock of varied origin, malarial infections and diverse intoxications from "anti-freeze" indulgence to sulfonamide therapy.

The unifying element in this heterogeneous complex, Acute Renal Failure, is renal ischemia, for under whatever clinical circumstance renal ischemia may develop, there the disruptive tubular lesions, either alone or mixed with the effect of antecedent toxic damage are found along with the functional disturbances of oliguria or anuria and all the consequent varied tubular dysfunctions that are revealed by clearance techniques. The syndrome remains, therefore, on the firm basis of an adequate correlation of its structural and functional characteristics an entity.

This unity, however, arises not from what happens primarily in the kidney but outside it in that area so susceptible of disturbance, the general circulation. What confronts us is therefore not a "renal disease" but a circulatory episode that may complicate any clinical situation and we therefore suggest that it be so recognized under some appropriate term.

In various clinical situations from the shock of crush injury to chemical intoxication, one may, on the development of this clinical-pathological syndrome, speak of the occurrence of an "ischemuric renal episode," thus emphasizing in a sort of portmanteau word the principal phenomenon of cause and effect, namely the renal ischemia and, to use an older term, the ischuria.

The structural status of the kidney in the case needs, or perhaps one might say deserves, no specific pathological designation, any more, for example, than the structural status of the kidney in chronic passive congestion is given a pathological-anatomical name.
With the unity of pathogenesis of the syndrome established and an appropriate term for its designation suggested, we can now turn to various problems regarding the correlation of the structural lesions with their functional and clinical manifestations. Perhaps the first of these that presents itself is the "cause" of the oliguria or anuria which is closely related to all those other more recondite disturbances that are revealed by clearance methods. It is surely too much to hope that they can all be elucidated, but it is encouraging to see that the earlier dichotomy of interpretation is progressively narrowing as both functionalist and morphologist gain in experience of the renal lesions and, it might be added, of each others' ideas.

From the morphological viewpoint it seems certain that there is no single "cause" of oliguria but a great collocation of them, or as we would prefer to put it, that a constellation or "field" of structural phenomena determines and at the same time is a consequent of the functional disturbances. This latter form of causal concept is particularly useful, because as will be evident in our listing of them, the "causes" are interdependent, one modifying, often intensifying and at times nullifying the other. Moreover, it is often impossible to say whether a certain structural change is the "cause" of a certain functional phenomenon or whether the latter is not the determinant of the former, for "structure" and "function" are not two separate categories of being but different aspects of the same thing (25). The morphologist who has no way of measuring quantitatively the many effects of either aspect of the lesion should, however, call attention to them, leaving the final decision of their relative importance or priority for future decision.

There can be little doubt that renal ischemia may result in decreased filtration in the earlier stages of the development of the syndrome. There is small likelihood, however, that the simplicity of this factor remains long uncomplicated. First of all, the visualization by the Schlegel technique of the patchy cortical ischemia which produces the tubular lesions is quite a different pattern than what might be suggested by the simple statement that cortical renal blood flow is reduced. As we have suggested, perhaps over-all renal blood flow is not greatly decreased and yet the patchy maldistribution of blood to nephrons 
may still result in tubular dysfunction and eventual structural damage. In any case the tubules under conditions of ischemia must suffer functional derangement and presumably one of the first and least effects, occurring long before the development of a structural lesion, would be a reduction in water absorption. At least this sequence of events is demonstrable in the perfused kidney of the frog (30), and so to the factor of reduced filtration is added a counter factor.

It would not seem likely that this period of the slightest of tubular dysfunctions can long endure; with the beginning of structural change an increased permeability of the tubule wall and backdiffusion begins. The methods of clearance have given nuch data at least for speculation in regard to the diffusion of the various substances through the tubule wall and the morphologist cannot be very helpful in the interpretation of this complex problem. He can, however, emphasize the importance of a much simpler form of tubule leakage to which functionalists have paid less attention, and this is the gross pouring of tubular fluid in toto through holes in disrupted tubules at every level of the nephron from the origin of the proximal convolution to the collecting tubule.

The leakage of tubule fluid into the interstitial tissue, a factor that might directly reduce the amount of fluid delivered to the collecting system, would seem to be one element in the production of the intertubular edema which may at times be so apparent in histological section. This edema in turn seems an important factor in the production of a rise in intrarenal pressure, a potential cause of oliguria by its pressure on tubules with a direct impedance of flow and on vessels, with an indirect effect in the same sense by its compression of thin afferent capillaries and a consequent exaggeration of renal ischemia and lessened filtration.

Moreover, the flooding of the interstitial tissue with tubule fluid produces much more than an increase in physical tension. In many instances, following the administration of sulfonamides, for example, leaking fluid is an irritant which produces an exudative inflammatory reaction which becomes granulomatous in its later development. The degree of this "pyelonephritis" varies remarkably in different kidneys for reasons that are not always apparent. Perhaps one constant factor in its production is the protein which the escaping tubule fluid contains, this derived from the increased permeability of glomerular membranes as a result of the original ischemic damage. And so another modification of another sort of intrarenal tension, the oncotic pressure of the interstitial fluid, results with effects on tubule resorption and blood flow that are as yet not clearly understood.

The interminable discussion and argument in the literature concerning the part played by stoppage of renal tubules with casts may well be the result of a confusion between the "casts" that are seen in the urine as discrete plugs and the accumulations of epithelial debris, pigment, and proteins in every degree of sol-gel transformation that occur in the tubule of the nephron. Much of this confusion would be avoided if it were remembered that, judging from the results of microdissection, it is very improbable that any cast seen in the urine could have come from a nephron: urinary casts arise in the straight collecting tubules from which they are readily expelled. It is hardly necessary to state that any physical obstruction whether in the form of solid matter or an increased viscosity of a semi-coagulated protein fluid will lessen tubular flow; or that a lessening of flow for this or another reason will tend to exaggerate accumulation of these impeding materials, which in their turn will further decrease flow. Moreover any impedance in flow through the lumen of a ruptured tubule, or one even more permeable than normal, will increase leakage into the interstitial tissue and so will result in alterations of physical and oncotic intrarenal pressure. Again a circular effect on tubule fluid flow results extending beyond its own immediate viscious circle, and by the consequent disturbance of renal blood flow enmeshing, wheels within wheels, one cycle of oliguria-producing phenomena with another.

If we have over-laboured in what is admittedly a highly speculative manner the discussion of the . question, "what is the cause of anuria," it is because one so frequently hears and even reads, where more considered treatment might be expected, argument brought either for or against some particular factor which doubtless seems to its proponent the definite, specific and therefore logically valid "cause." A great deal of such para-logical discussion we believe originates in the 
subconscious use of a "linear" concept of causality" and would never arise if the constellational or field concept were applied to the eluciclation of what must surely be an infinite collocation of related phenomena among which we can at best define only a few. ${ }^{10}$

A few other clinical correlations nay be briefly attempted. During the stage of recovery the volume of urine increases, at times rather suddenly, to a degree that might be considered a state of diuresis. In its explanation a morphologist can do little but draw on an imagination reasonably controlled by what he knows of the altered structural status of the kidney: certainly it is difficult for him to believe that any profound structural change has abruptly occurred in the danaged kiclney, such as a sudden flushing out of impecling casts and clebris from tubule lumens, for these structures have too much the look of permanency in his dissected specimens. What seems more likely to him is that a partial recovery of both blood flow and tubule function has occurred to that level where a moderate filtration is combined with a still inadequate tulular absorption of water, a situation mentioned earlier as a theoretical step in the progressive development of the functional lesion of oliguria. Under such conditions a very little filtrate from a few nephrons might result in a large volume of urine and such a change, being deternined by increased blood flow, might develop suddenly as it depends on no alteration of the structural basis of the renal lesion but rather on a relaxation of vascular spasm.

To speak of reparative processes raises the question of the very considerable practical importance in problems of prognosis, prophylaxis

\footnotetext{
10 In this last regard, that is, our doubtless long to continue ignorance of the totality of renal fundamentals, it is well to remember that in the present problem, the explanation of a change in rate of flow through the renal tubules, we do not yet know how fluid cain flow through the tubule even under the presumably relative simpler conditions that obtain in the normal nephron! Lichtwitz (31) was forced to the assumption that tubule fluid, like mercury in contact with glass, does not wet the wall of the tubule and Winton (32) after considerable calculation leaves the mystery with the pertinent suggestion that, in view of our uncertainty regarding this basic datum, we need feel no surprise if our explanations of its infinitely more complicated derivative problems prove inadequate.
}

and of treatment, of what may have seemed to be largely an excessive refinement, namely the sharp distinction we have macle between the two sorts of tubular damage. As we have shown, the repair of extensive and severe disruptive tubulorhextic lesions is difficult of achievement; extensive but futile "regeneration of tubular epithelium" may occur with nevertheless a failure of reconstitution of nephrons whose structure is compatible with function. Nephrons so affected are probably lost forever and a return to adequate renal function must therefore come from compensatory processes in those that escaped the original ischemic insult. The number of these is cloubtless determined in the early stages of the syndrome, so that the sooner renal blood flow is increased the better the chance of ultimate recovery. After the ischemuric episode has occurred and its disruptive lesions are established it would seem doubtful if any amount of renal circulatory improvement could do much good. The clinical instance of glycol intoxication which we have described, where after three weeks there had been no return to functional normality even with a reestablished "urinary output," is a good example. This is not true of those kidneys where the nephrotoxic tubular lesions predominate; here the intact basement membrane provides the scaffolding for epithelial regeneration and nephron reconstitution; if the individual can be maintained a sufficient time. recovery of a functioning kidney can occur although the great majority of the nephrons were originally damaged. Unfortunately, as our cases of glycol and sublimate poisoning i1lustrated, severe intoxication leads to the complication of a renal ischemia with added disruptive tulular damage, so that the prognosis in such instances is perhaps not better or may be even worse than in the simpler case of the "crush kidney."

Complication by an ischenuric episode with its disruptive tubular damage in cases of poisoning may well be a reason for the failure of such therapeutic agents as BAL in certain specific forms of poisoning and in any example of severe intoxication; no matter how efficacious this procedure may be in its effect on the nephrotoxic action of the poison, it seems clear that its mechanisms cannot obviate or relieve the effect of the complicating renal ischemia and its consequent disruptive tubular damage. A reference to the 
case of sublimate poisoning treated with this agent will emphasize this point.

It may seem to some that a defect in our final conclusions is our inability, or disinclination, to give a specific anatomical-pathological name to the abnormal kidney in Acute Renal Failure. We believe, however, that the situation in the kidney, even from the relatively simpler aspect of its structural alteration, is much too complex to be covered by any meaningful term. A recent attempt to do so illustrates this difficulty: the conditions with which we are concerned are distributed variously among three categories, Upper $\mathrm{Ne}$ phron Nephrosis, Distal Nephron Nephrosis and, apparently to catch the anomalous stragglers, Upper and Lower Nephron Nephrosis. It would seem unlikely that any sort of renal lesion could escape this nosological net, but what does one distinguish by the use of such all-inclusive terms? Only that there seems to be no Middle Nephron Nephrosis.

The structural lesions, if not the sort of "renal disease." that occur in Acute Renal Failure can, however, be accurately and meaningfully stated: they are: "nephrotoxic tubular necrosis" and disruptive tubular damage or "tubulorhexis," each with its distinctive structural characteristics both in regard to the sort of damage that is done to the tubule and to the location of the lesions. Of perhaps more importance to the clinician, each has its differential aetiological characteristic, a renal poison in the one case, and in the other the ischemia of the ischemuric episode. All these essential connotations are clouded or even completely obscured when an attempt is made to find a phrase that covers everything and describes nothing.

It is, therefore, when one attempts to pass from the objective level of the renal lesions to the higher abstraction of a "renal disease" that semantic difficulties with all their conceptual confusion result. The answer, of course, is not to take this unnecessary step, for Acute Renal Failure is the result not of a specific sort of altered kidney that can be given a name, but of the summation of various structural-functional lesions in several million nephrons (19).

It is quite true that under controlled experimental conditions Acute Renal Failure can be produced in two specific forms, one characterized by purely nephrotoxic tubular lesions limited to the proximal convolution or on the other hand, as our dissections of the material of Van Slyke, Phillips and their co-workers showed, a contrasting renal status with only disruptive tubulorhextic lesions so pure in form that even the mitochondrial elements of the intact tubule showed no evidence of toxic effect.

It seems very doubtful, however, if the clearcut distinctions of the experimental situation ever occur spontaneously in Acute Renal Failure as it happens in man. The "crush kidney," or the kidney from individuals where an ischemuric episode has occurred during surgical shock, may approach the ideal example of purely tubulorhextic damage, but in the protracted course of the renal failure it will be the rare case where some conplicating nephrotoxic damage to the renal tubules, such as the "cloudy swelling," has not occurred.

In the fatal case of Acute Renal Failure where an ischenuric episode has complicated poisoning, the abnormal structural renal status is from its origin always confused; there are both nephrotoxic tubular lesions peculiar to the specific poison in the proximal convolution and the disruptive tubular lesions of renal ischemia everywhere in the nephron, all so mixed and blended that their separate identities are at times difficult to distinguish even in the continuity of a single isolated nephron. Moreover, besides necrosis of tubules there are present the interstitial inflamnatory reaction that follows tubular disruption and the abnormalities in the epithelial regenerative processes with the failure of restitution of functioning nephrons.

From the standpoint of pathogenesis, one phenomenon stands clear in this confusion of various tubular necroses, inflammatory reactions and regenerative abnormalities-the ischemuric episodefor the occurrence or not of this event determines the course and direction of the reactions in the kidney to the ill effects of trammatic and toxic injury.

\section{CONCLUSIONS}

In Acute Renal Failure related to toxic and traumatic injuries the structural alterations which are correlated with and which may reasonably explain the functional disturbances in the kidney are those of a tubular nephropathy. 
Two sorts of tubule injury are encountered. The first is a nephrotoxic necrosis limited to that part of the nephron, the proximal convolution, which is functionally concerned with the handling of the poison. Since poisons are distributed by the renal circulation all nephrons are equally involved. The second type of lesion is a disruption of the renal tubule (tubulorhexis) due to focal cortical ischemia. It occurs at random among nephrons and in any part of a nephron.

In the kidney of any case of fatal Acute Renal Failure arising under various clinical circumstances these two lesions appear in varying proportions depending on the nature of the renal insult, whether toxic or circulatory or both. In Acute Renal Failure of non-toxic origin (shock, crush injury, etc.) the insult is purely circulatory, a part of or an episode in the general circulatory disturbance which has involved the peculiarly susceptible area of the renal blood flow. The term ischemuric episode is suggested to designate this complication.

Such a renal circulatory episode also may occur as a consequent of the general circulatory disturbances of grave intoxication. From this coincidence stems the unity of pathogenesis in the group of Acute Renal Failure as a whole the members of which include such clinically disparate origins as crush injury, transfusion reactions, obstetrical accidents and fatal poisonings.

\section{REFERENCES}

1. Smith, H., The Kidney; Structure and Function in Health and Disease. Oxford Univ. Press, New York, 1951.

2. Schlegel, J. U., Demonstration of blood vessels and lymphatics with a fluorescent dye in ultraviolet light. Anat. Rec., 1949, 105, 433.

3. Lucké, B., Lower nephron nephrosis (renal lesions of the crush syndrome, of burns, transfusions, and other conditions affecting lower segment of nephrons). Mil. Surgeon, 1946, 99, 371.

4. Mallory, T. B., Hemoglobinuric nephrosis in traumatic shock. Am. J. Clin. Path., 1947, 17, 427.

5. Van Slyke, D. D., The effects of shock on the kidney. Ann. Int. Med., 1948, 28, 701.

6. Corcoran, A. C., and Page, I. H., Crush syndrome: post-traumatic anuria. J. A. M. A., 1947, 134, 436.

7. Burch, G. E., and Ray, C. T., Lower nephron syndrome. Ann. Int. Med., 1949, 31, 750.

8. Dunn, J. S., Gillespie, M., and Niven, J. S. F., Renal lesions in two cases of crush syndrome. Lancet, $1941,2,549$.
9. Oliver, J., The structure of the metabolic process in the nephron. J. Mt. Sinai Hosp., 1948-49, 15, 175.

10. Walker, A. M., and Oliver, J., Methods for the collection of fluid from single glomeruli and tubules of the mammalian kidney. Am. J. Physiol, 1941, 134, 562.

11. Greenspan, E. M., Hyperchloremic acidosis and nephrocalcinosis. The syndrome of pure "lower nephron" insufficiency. Arch. Int. Med., 1949, 83, 271.

12. Oliver, J., New directions in renal morphology: a method, its results and its future. Harvey Lect., 1945, 40, 102.

13. Oliver, J., Architecture of the Kidney in Chronic Bright's Disease. Paul B. Hoeber, New York, 1939.

14. Susuki, T., Zur Morphologie der Nierensekretion unter physiologischen und pathologischen Bedingungen. G. Fischer, Jena, 1912.

15. Edwards, J. G., The renal tubule (nephron) as affected by mercury. Am. J. Path., 1942, 18, 1011.

16. Bergstrand, A., and Bergstrand, H., The pathology of glomerulo-nephritis and related diseases. Scandinav. J. Clin. \& Lab. Invest., 1949, 1, 334.

17. Muller, F., Bezeichnung and Begriffsbestimmung auf dem Gebiet der Nierenkrankheiten. Veroeflentl. a. d. Geb. d. Mil. San. Wes., 1917, 65, 21.

18. Rather, L. J., Renal athrocytosis and intracellular digestion of intraperitoneally injected hemoglobin in rats. J. Exper. Med., 1948, 87, 163.

19. Oliver, J., When is the kidney not a kidney? J. Urol., $1950,63,373$.

20. Sussman, R. M., and Kayden, H. J., Renal insufficiency due to paroxysmal cold hemoglobinuria. Arch. Int. Med., 1948, 82, 598.

21. Schlegel, J. U., and Moses, J. B, A method for visualization of kidney blood vessels applied to studies of the crush syndrome. Proc. Soc. Exper. Biol. \& Med., 1950, 74, 832.

22. Phillips, R. A., Dole, V. P., Hamilton, P. B., Emerson, K., Jr., Archibald, R. M., and Van Slyke, D. D., Effects of acute hemorrhagic and traumatic shock on renal functions of dogs. Am. J. Physiol., 1945-46, 145, 314.

23. Foote, J. J., and Grafflin, A. L., Quantitative measurements of the fat-laden and fat-free segments of the proximal tubule in the nephron of the cat and dog. Anat. Rec., 1938, 72, 169.

24. Lippman, R. W., Ureen, H. J., and Oliver, J., Mechanism of proteinuria. III. A comparison of the functional and structural aspects of the effects of certain intraperitoneally administered proteins on hemoglobin excretion in the rat. J. Exper. Med., 1951, 93, 325.

25. Oliver, J., When seeing is believing. (Festschift for Thomas Addis.) Stanford M. Bull., 1948, 6, 7.

26. Sirota, J. H., Carbon tetrachloride poisoning in man. I. The mechanisms of renal failure and recovery. J. Clin. Invest., 1949, 28, 1412. 
27. Marshall, D., and Hoffman, W. S., The nature of the altered renal function in lower nephron nephrosis. J. Lab. \& Clin. Med., 1949, 34, 31.

28. Oliver, J., The histogenesis of chronic uranium nephritis with especial reference to epithelial regeneration. J. Exper. Med., 1915, 21, 425.

29. Oliver, J., An essay towards a dynamic morphology of the mammalian nephron. Am. J. Med., 1950, 9, 88.
30. Oliver, J., Experimental nephritis in the frog. IV. The significance of the functional response to vascular and to parenchymal disturbances in the kidney. J. Exper. Med., 1932, 55, 295.

31. Lichtwitz, L., Die Praxis der Nierenkrankheiten. J. Springer, Berlin, 1934, 3. Aufl.

32. Winton, F. R., Physical factors involved in the activities of the mammalian kidney. Physiol. Rev., $1937,17,408$. 


\section{Plate 1}

This figure shows the detail that can be seen in a normal nephron (rabbit) when fixed immediately in 10 per cent formalin, dissected after maceration in concentrated $\mathrm{HCl}$ and stained with iron haemotoxylin, mounted in $\mathrm{H}_{2} \mathrm{O}$ without the use of any clearing agent and photographed through the thickness of the specimen $(60 \mu)$ at a magnification of $100 \times$.

The nuclei are unstained and therefore appear as clear spots on the dark mitochondrial pattern of the renal epithelium. The decreasing gradient of the mitochondrial pattern is well shown, so dense in its first portion as to almost obscure the nuclei and fading almost to disappearance in the terminal medullary portion.

Certain discrepancies may be noted in the size of the objects photographed in the following plates. This is due to the pressure of the cover slip which flattens and spreads the glomeruli, actually $0.20 \mathrm{~mm}$. in diameter, so that they appear out of proportion to less compressed tubules. 


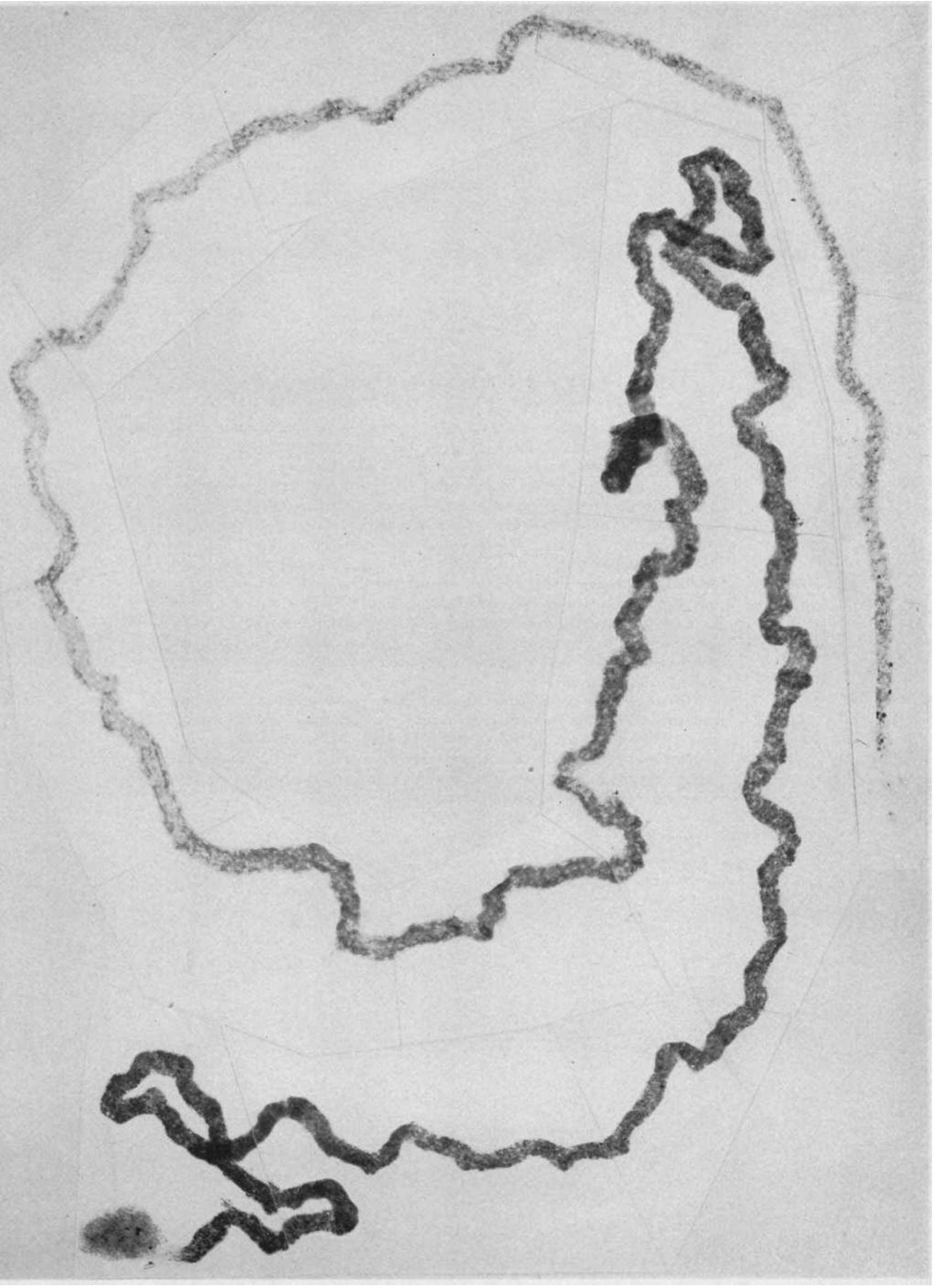




\section{Plate 2. Defails of the Preceding Convolution of Plate 1 at a Magnification of $300 \times$. (See Legend to Plate 1)}

In $\mathrm{A}$ to $\mathrm{E}$ are portions of the convolution showing the decreasing gradient of the mitochondrial apparatus, so dense near the glomerulus as to obscure the nuclei (A) and practically invisible in the medullary portions (D) and (E). In favorable regions of focus and where not too densely packed together the basal ends of the rodlets can be seen at the arrows. In the midportion of the convolution where the mitochondria are less dense the brush border can be seen (C). The ascending limb is shown in $F$ and in $G$ the distal convolution with its stout heavy rodlets. In $\mathrm{H}$ is the transition of the proximal convolution into the thin, descending limb of Henle's loop. The tubule is over-stained to show the islands of cells in the proximal convolution as they progressively fade into the capillary-like thin segment. The diverticulum on the proximal convolution (A) is the only one we have ever noted in any species in this situation; they are common in the distal convolution of man (Plate 27).

The appearances noted above are only perfectly seen in immediately fixed material: 20 minutes delay at room temperature will cause a dissolution of the mitochondrial rodlets to a diffuse granular background. As in the histological section, human autopsy material therefore never shows the perfect picture of these finer structures; this contrast will be apparent in a comparison of the human with the experimental material. 


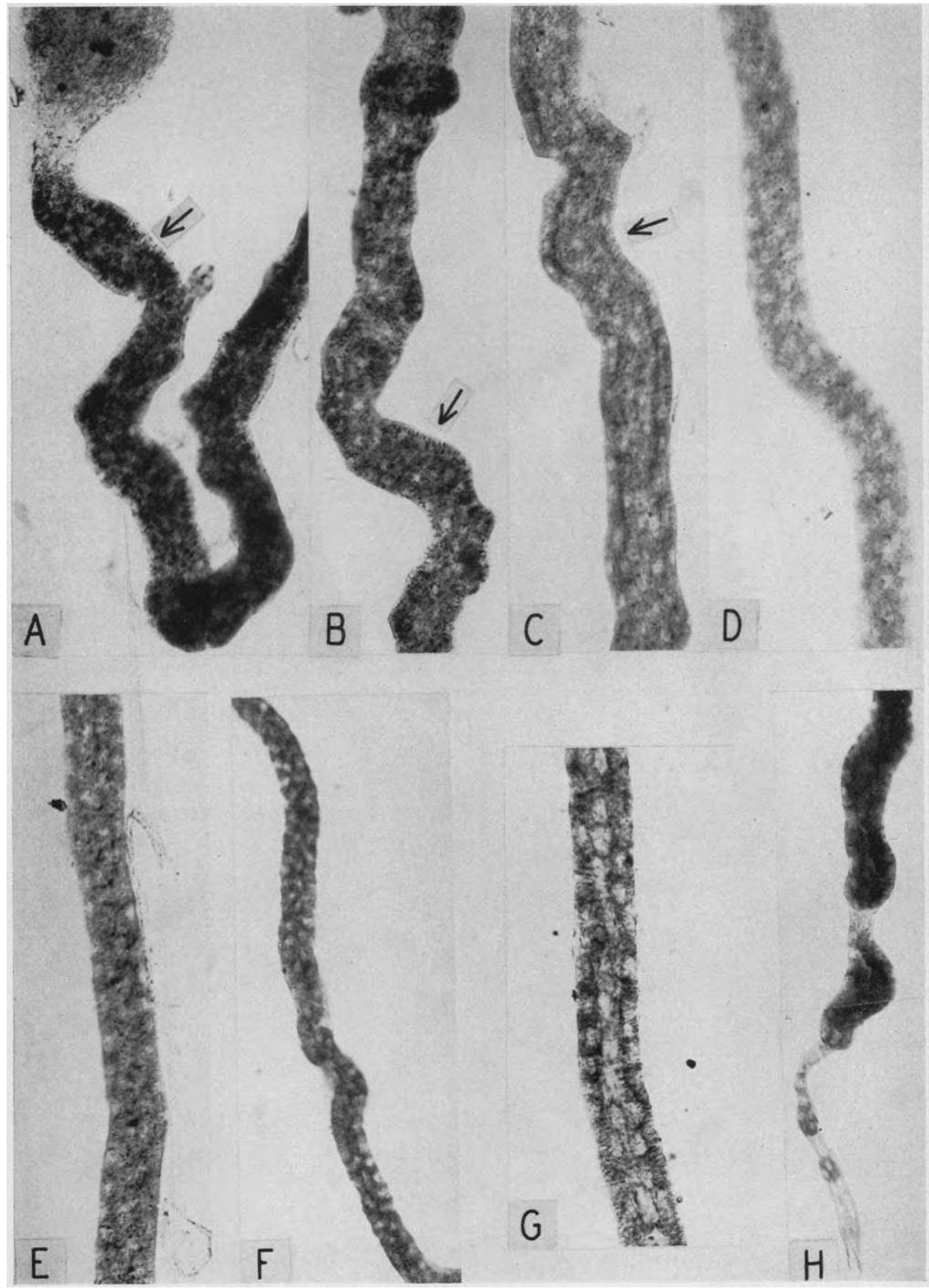


Plate 3. Crush Anuria-Man Buried lnder Debris for Several. Holrs

Fracture of spine with compression of cord; passed a few oz. of urine each 24 hours and died with a blood urea of $420 \mathrm{mg}$. per cent ( $c f$. J. Path. \& Bact., 1941, 53, 493, Case 1). Specimen through the courtesy of Dr. J. E. Morison, Belfast, N. I.

A complete proximal convolution with glomerulus. The patchiness of the staining of the mitochondrial pattern is evidence of the damage to the tubular epithelium. At the arrows this danage is so intense as to have resulted in disruption of the basement membrane and the epithelium is reduced to granular debris. In spite of these areas of tubular destruction, note intervening stretches with normal nuclei and mitochondrial apparatus (A). Areas of damaged and well preserved tubule alternate irregularly to the terminal medullary portion of the convolution (B). Here the basement membrane is barely visible and there is complete destruction of the renal epithelium; the irregular black stained spots are clumps of cellular debris and detritus. Magnification $80 \times$.

The insert shows the detail of an incipient disruptive lesion. Magnification $200 \times$. 


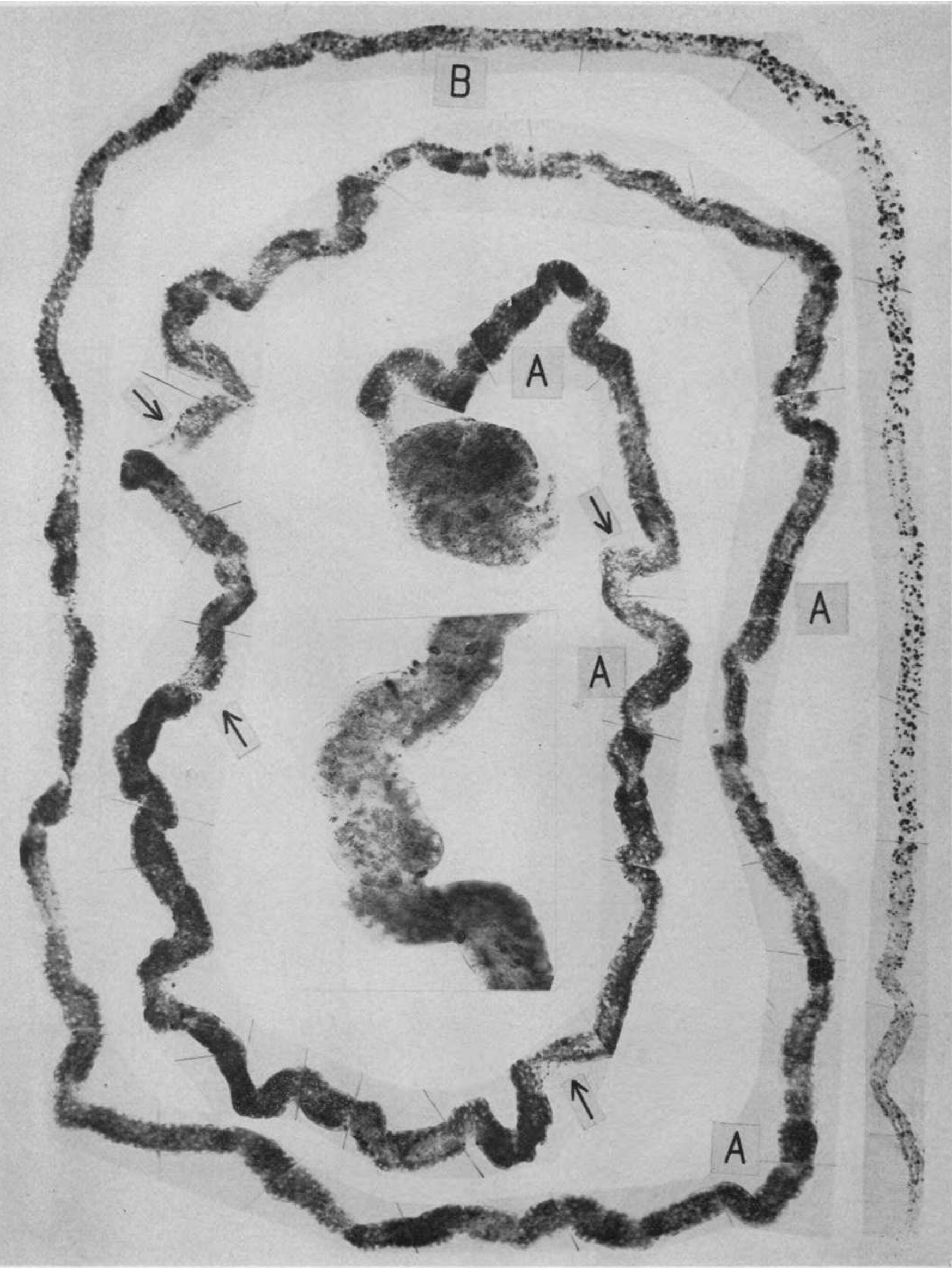




\section{Plate 4. A Spray of Distal Convolutions Jolning to Form a}

Collecting Tubule from the Same Kidney as in Plate 3

Four ascending limbs with normal nuclear pattern and tubular contour rise on the left to pass into the distal convolutions. Note the rupture of the basement membrane and tubular disruption in all four at the arrows; each distal is distended with a heme pigment cast (A) of varying length; note lack of cast or pigment at points of tubular damage and the lack of disruption where the casts distend the tubule. The cast is continuous in the upper two distals far into the collecting tubule. Magnification $40 \times$.

Insert shows detail of disruptive lesion in lower distal convolution. Magnification $100 \times$. 


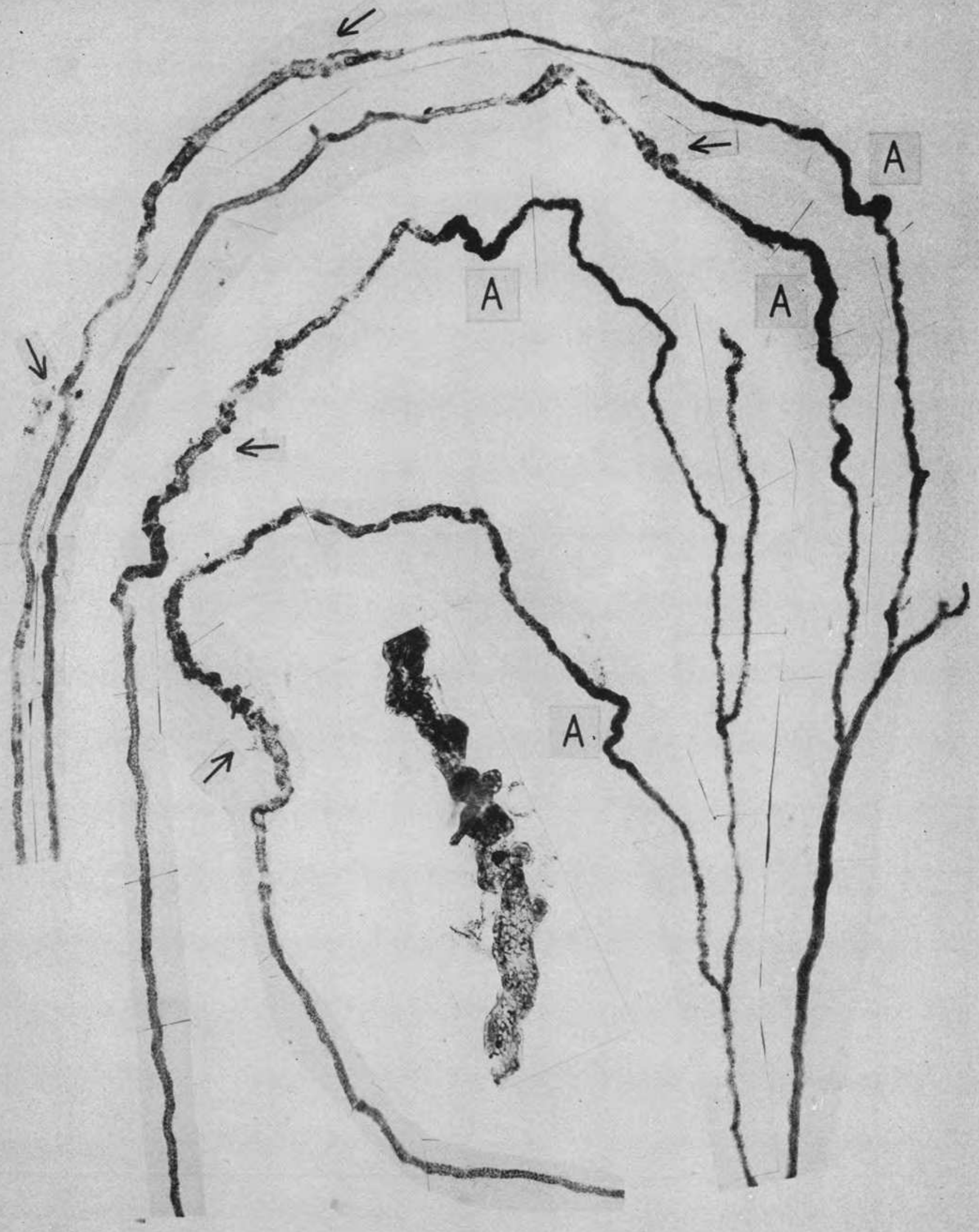


Plate 5. Crush Antria-Young Woman Pinned under a Beam for Six Hours

Legs crushed; shock. Lived nine days, urinary output 120 to $420 \mathrm{cc}$. per 24 hours, terminal blood urea 368. (Cf. Lancet, 1941, 2, 549, Case 1). Specimen through the courtesy of the late John Shaw Dunn of Glasgow, Scotland.

The appearance of the disruptive lesion in the distal convolution from the first case in which it was described by Dunn, Gillespie and Niven (8). An ascending limb with a fairly well preserved nuclear pattern curves down and then up to the distal convolution. At this point (A) the basement membrane is ruptured and the tubular wall disrupted. There is no heme cast at this point of damage but at $B$ the tubule is distended with a black pigment cast and there is an extensive fragmentation of the tubule wall. Surrounding the fragmented tubule are granulomatous adhesions in which large capillary venous channels can be seen. Another fragmentation follows C; irregular masses of regenerating epithelial cells can be seen in the tubule below this point (D) but the tubule wall is not repaired. Magnification $81 \times$. 


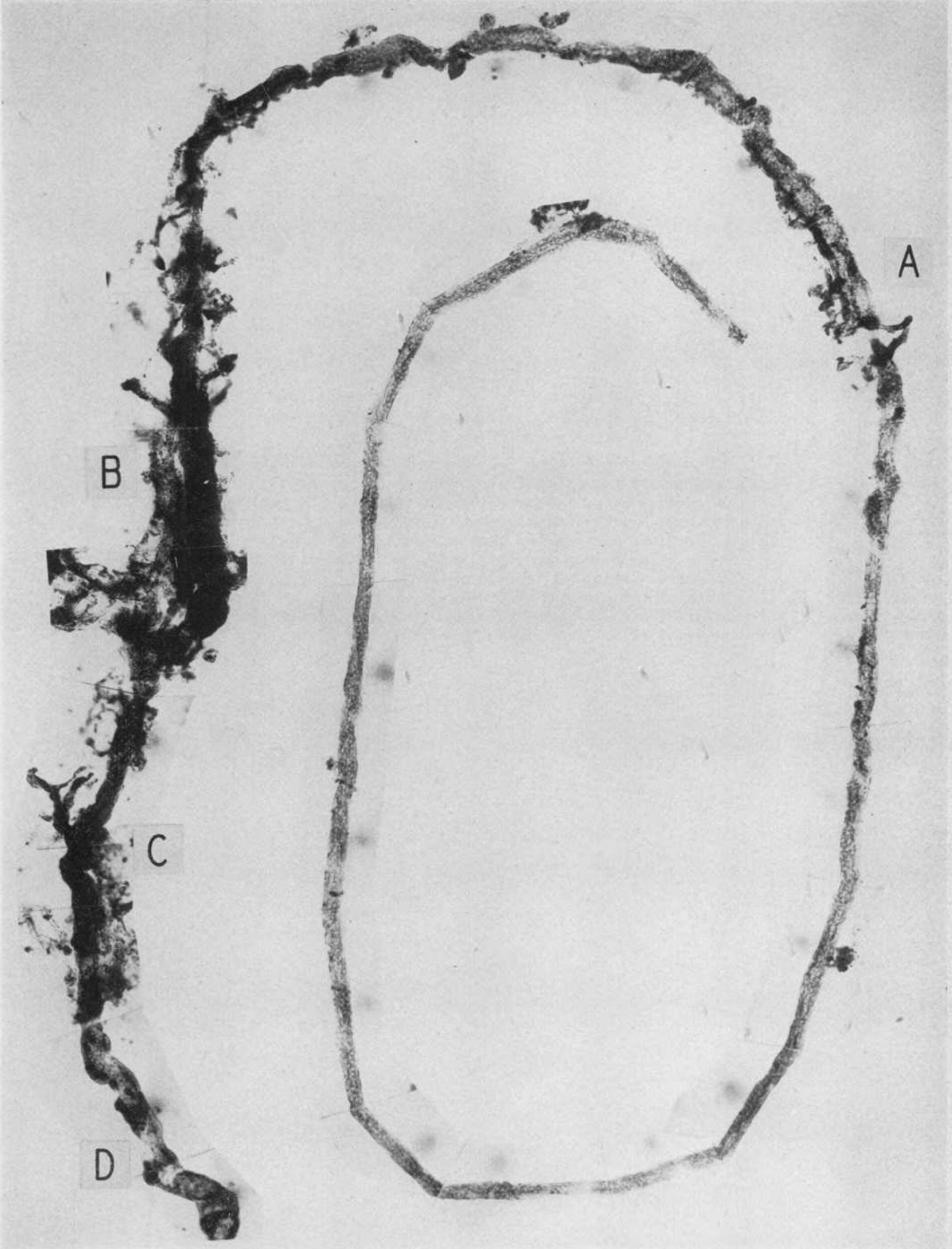


Plate 6. Fatal Burns-Man Sustained Multiple Third Degree Burns OVER $1 / 6$ OF HIS BODY

Urinary output $600 \mathrm{cc}$. per day until death on ninth day. Specimen through the courtesy of Dr. Baldwin Lucké.

A complete proximal convolution with its glomerulus. In the first quarter of the convolution the nuclear and mitochondrial pattern is essentially normal; from this point on there is extreme damage. At A the tubule is swollen, the basement membrane broken, the renal epithelium necrotic, and the tubular wall disrupted. Varying degrees of tubular damage alternate until the terminal portion of the convolution is reached (B); the basement membrane shows almost complete dissolution and only irregular clumps of nuclear and cellular debris remain. Magnification $83 \times$. 


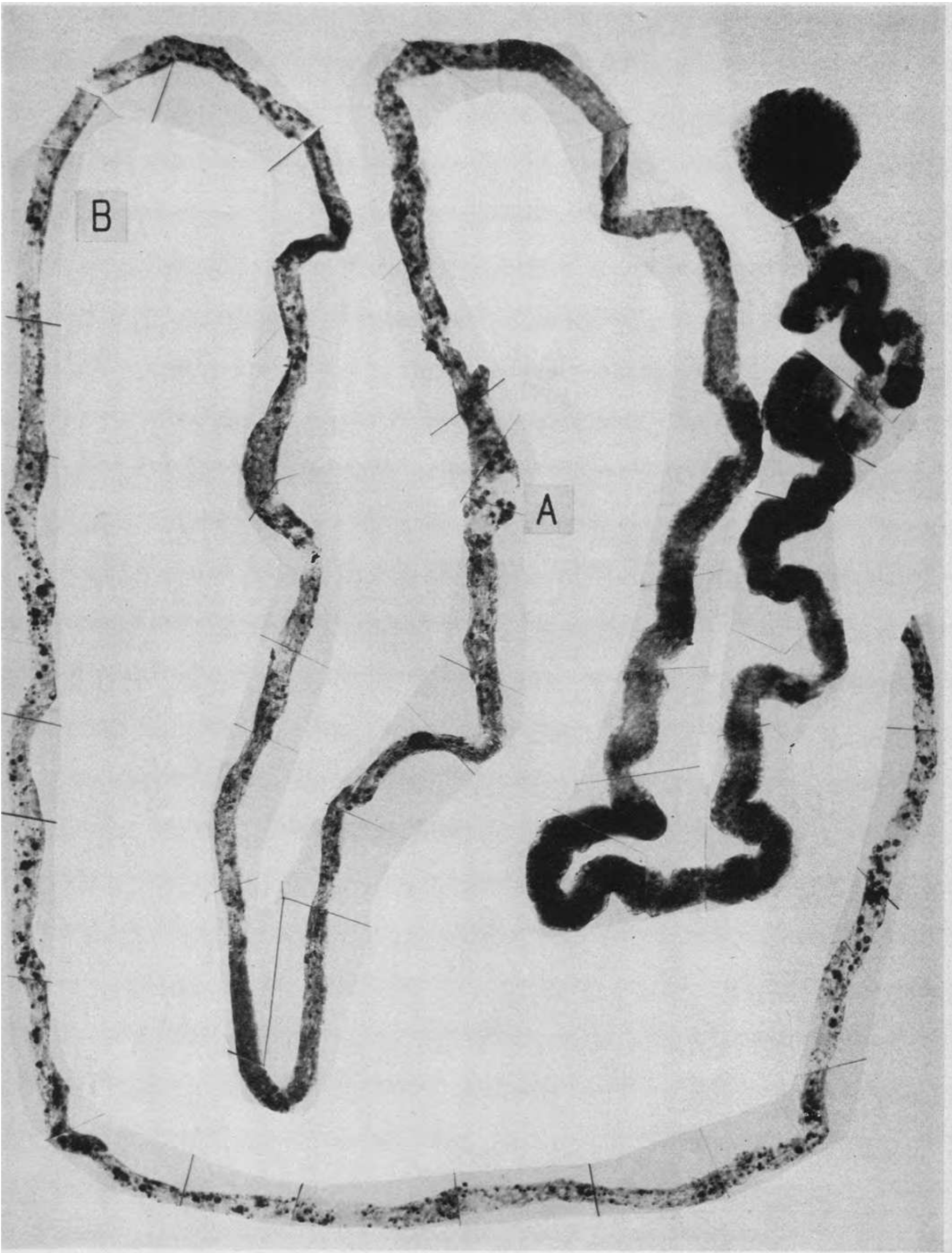


Plaie 7. The Ascenuing Limb and Distal Convolution of the Same Nephron as in Plate 6

The former curves from right to left: it is fairly well preserved in its first portion but from A its basenent membrane is fragmentary and the epithelium necrotic and desquamated. In the distal convolution there is a segment of tubular disruption with fragmentation of the basement membrane (B); note that there is no associated heme cast or deposit in the tubule lumen. Where these objects occur (C) there is no tubular disruption.

The damaged terminal segment of the proximal convolution shown in the preceding figure and the ascending limb of this figure lay side by side in the subcortical medulla. It is commonly stated that the distinction between them in the histological section is not difficult, an opinion which may be judged by comparing the two figures. Magnification $83 \times$.

Insert shows detail of disruptive lesion at a magnification of $200 \times$. 


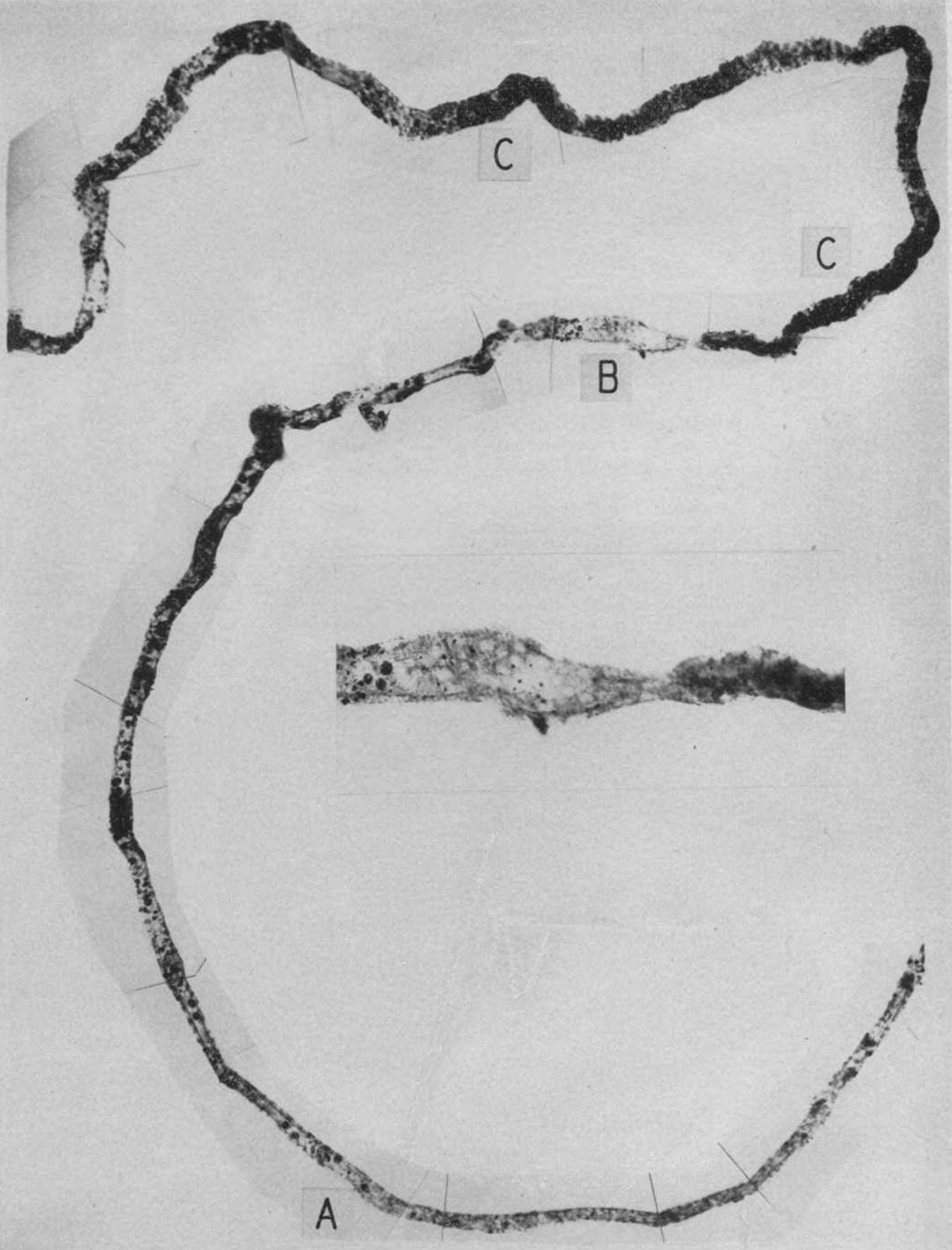


Plate 8. Fatal Burns-Man Burned by Gasoline Explosion, Face, Neck, Shoulders and Back to Waist

Died in renal failure on ninth day. Specimen through the courtesy of Dr. N. Winer.

The insert shows a Camera lucida tracing of a complete nephron. The cross-hatched portion of the tubule indicates the seat of extreme tubular disruption as illustrated in the photographs. As evidence of the random distribution of the disruptive lesions note that the terminal segment of the proximal convolution was intact in this specimen. The distal convolution contained no heme casts and showed no apparent lesions. In Inserts 1, 6-8 the nuclear patterns are normal, but obscured by the dense black staining of the mitochondrial material. Magnification $83 \times$. 


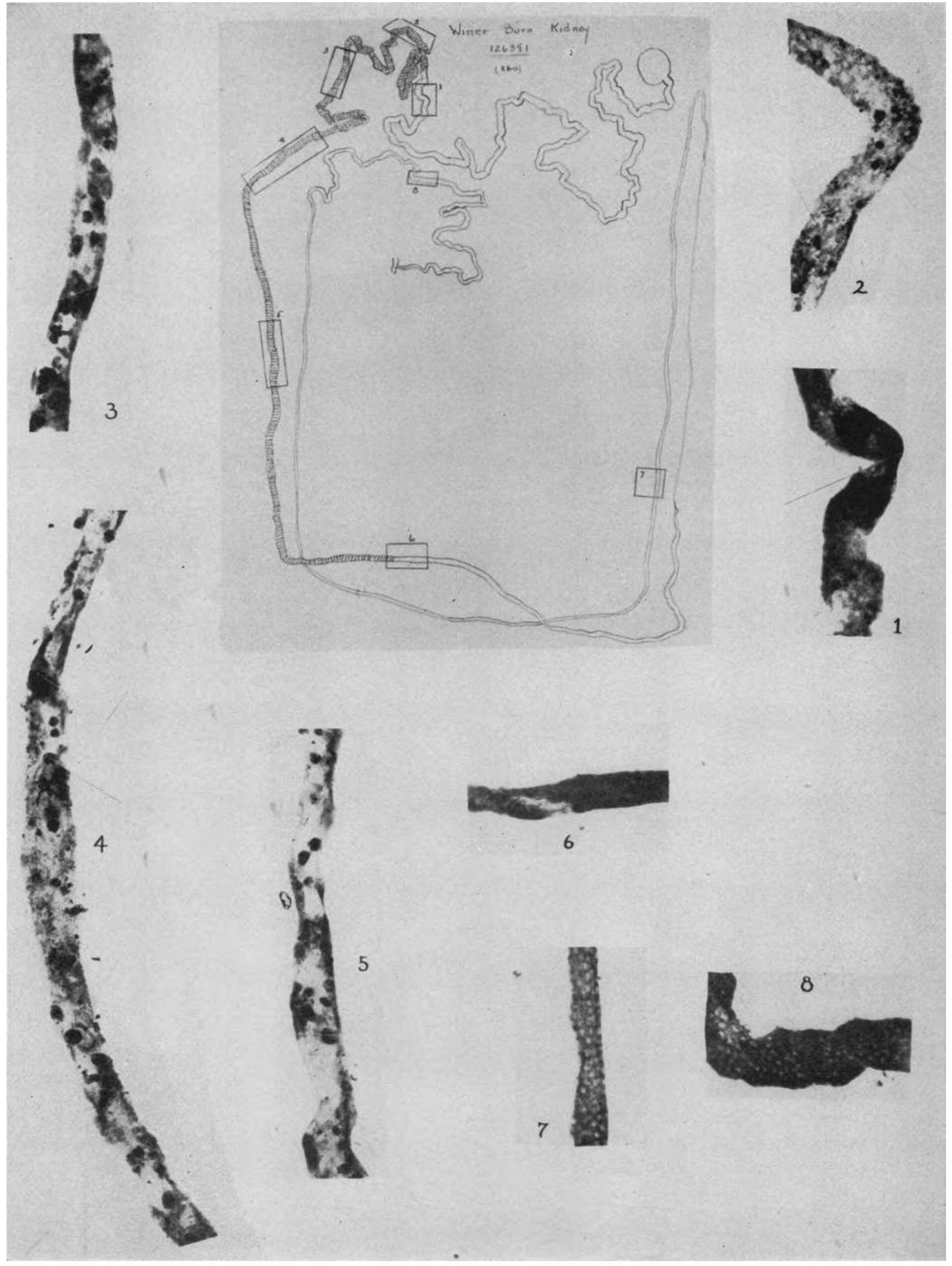


Pi.ate 9. Uterine Hemorrhage-Copiols Hearorrhage in

Third Stage of Labor; Shock

Plasma, whole blood, and haemoglobin solution given intravenously. Urine volume on fourth day $120 \mathrm{cc}$; $360 \mathrm{cc}$. on the sixth. Died on the ninth day. Specimen through the courtesy of Dr. Baldwin Lucké.

Glomerulus and proximal convolution. The greater part of the proximal convolution is well preserved: the heavy staining was deliberately done to bring out the contrast of two regions (A) where stretches of the convolution show damage. In the more proximal a slight disruptive lesion is shown; in the second there is complete disruption of the tubule wall with surrounding tissue reaction. Magnification $83 \times$.

Insert shows incipient disruptive lesion. Magnification $200 \times$. 


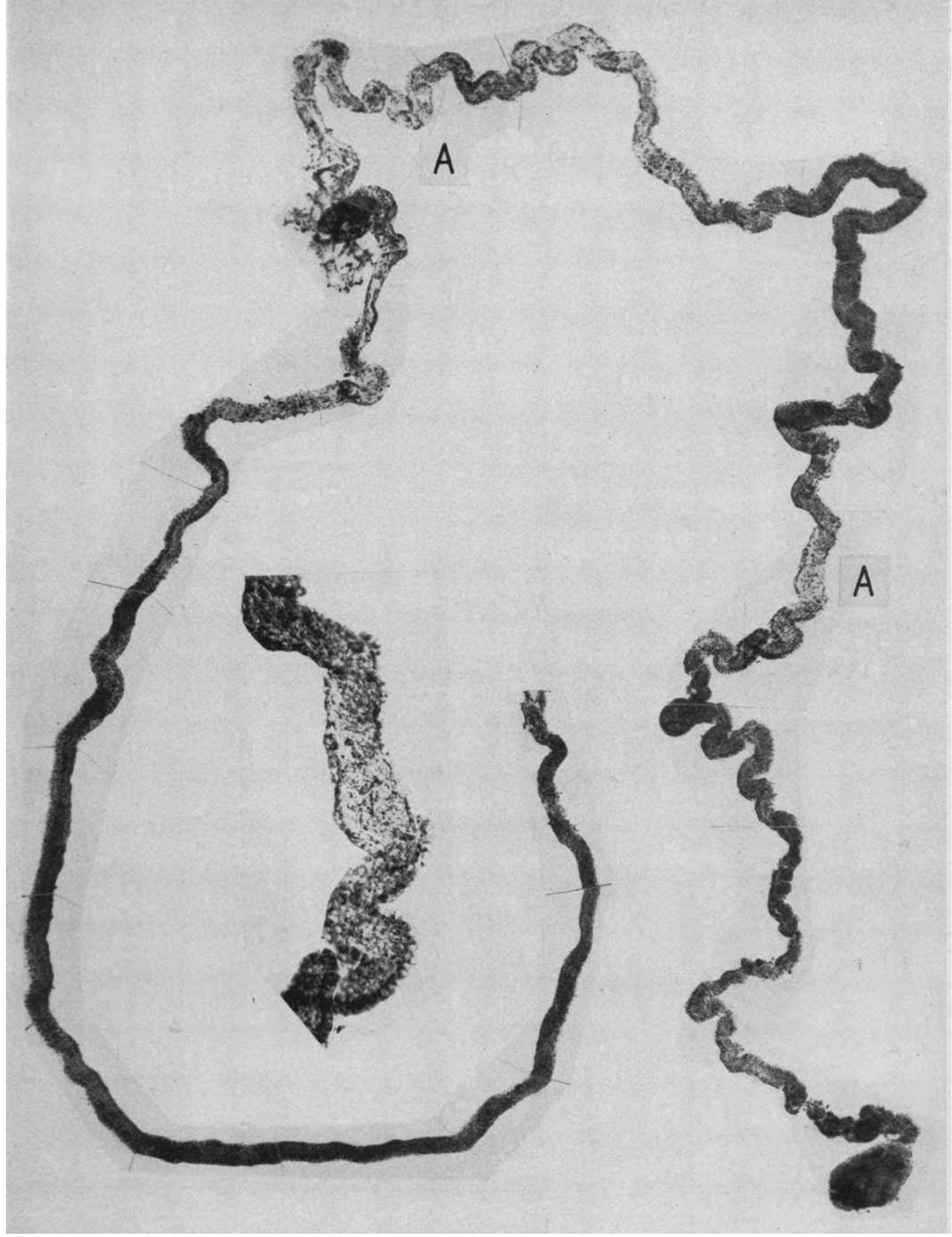


Plate 10. Ascending Limb and Distal Convolution from the Same Kidney as in Plate 9

The first portion of the ascending limb is intact, from $\mathrm{A}$ it shows extensive damage. At $\mathrm{B}$ is complete disruption of the distal convolution with surrounding adhesions of reactive granulation tissue in which are large capillary-like channels. The dense black segments of the greater part of the convolution are tubule filled with heme cast ; as is so commonly the case there is no apparent lesion in the tubule in the proximity of the pigment casts. Magnification $84 \times$.

Insert shows detail of disruptive lesion at a magnification of $200 \times$. 


$$
5
$$


Plate 11. Transfusion Reaction-Transurethrai. Prostatectomy, SHOCK

B.P. 78/60. Three blood transfusions. Urine output from retained catheter not measured. Died on fifth day following operation with "marked elevation of blood chemistry."

A complete proximal convolution. The glomerulus is missing. The most striking feature shown by this specimen is the random scattering of stretches of disrupted tubule (A) among portions in which the nuclear pattern indicates a relative normality of the tubule wall. (B) The very dark stretch of tubule is due to the presence of haemoglohin in the renal epithelium. Magnification $82 \times$. 


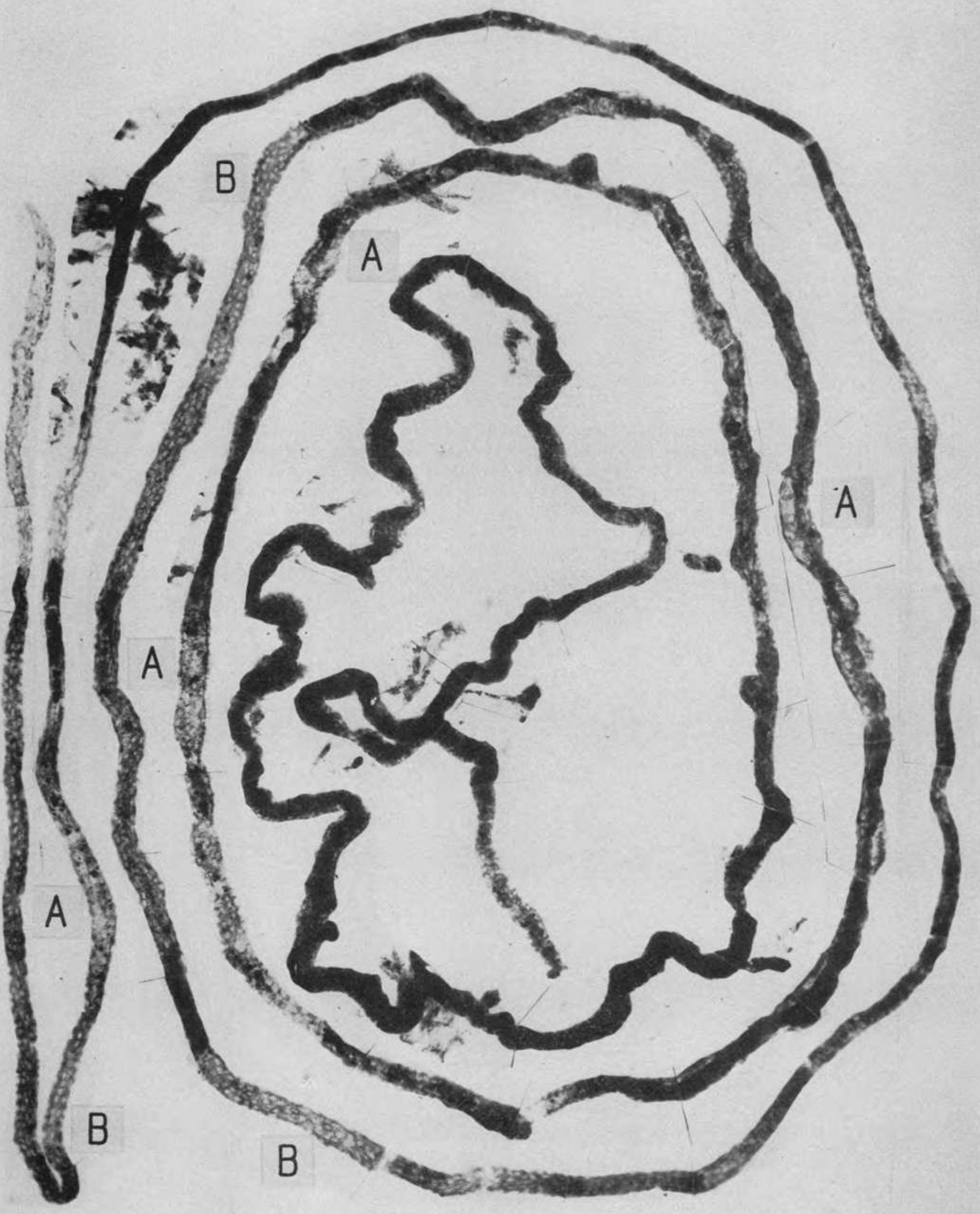


Plate 12. The Terminal Segment of Axother Proximal Convolution from the Same Kidney as in Plate 11

Regeneration has replaced the original epithelium by cells with oval nuclei and with sparce mitochondrial material. The new epithelium had absorbed haemoglobin as is evident from the dark granules which were positive for free iron with the Berlin Blue reaction. At A debris impregnated with blood pigment, negative for free iron but positive to benzidine, fills and distends the tubule lumen. Note that as is usual, these substances have caused no damage to the epithelium of the tubule wall. Magnification $82 \times$.

Insert shows detail of pigment in regenerated cells at a magnification of $200 \times$. 


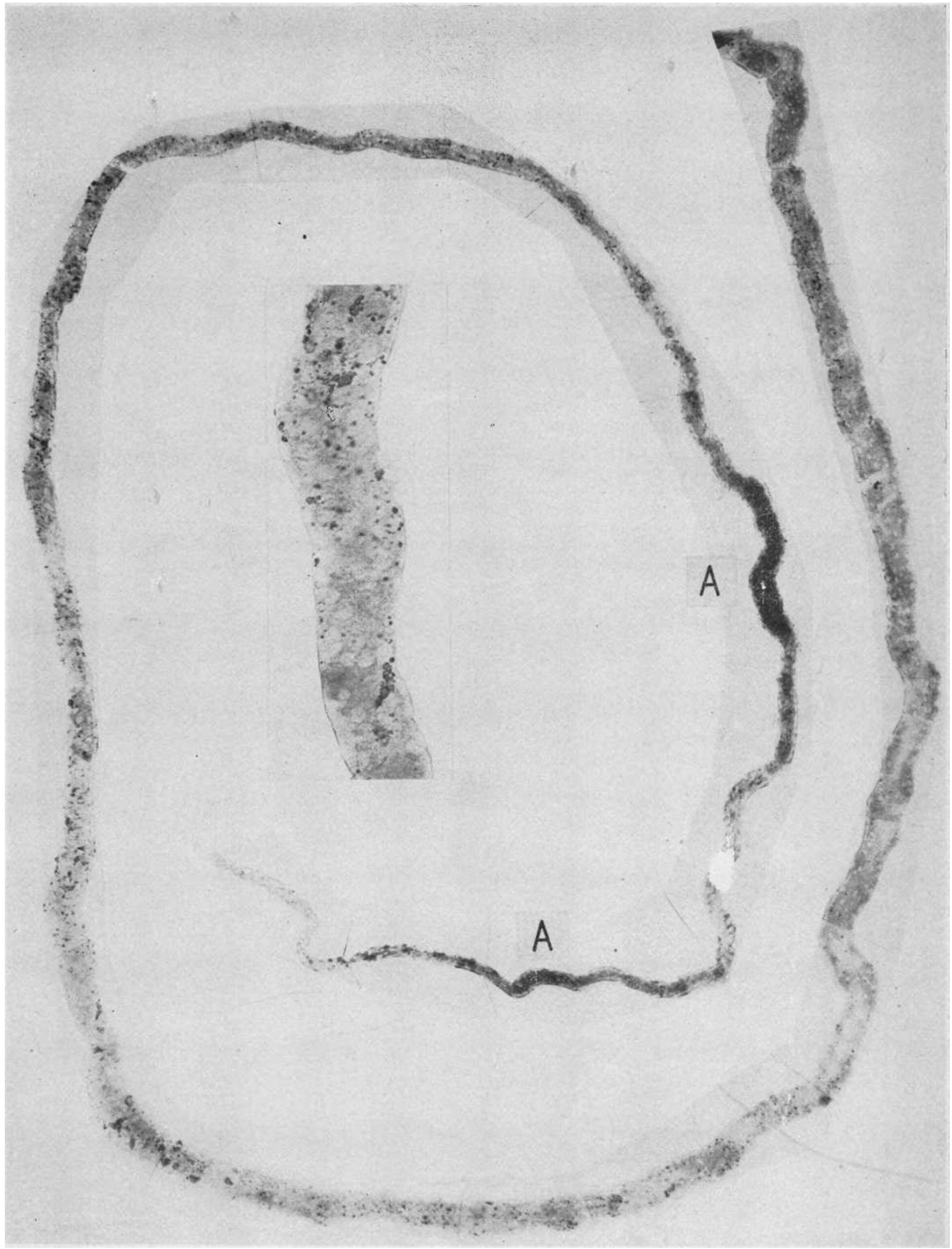


Piate 13. Blackwater Fever-Man Brolgint from the Colntrysine with a Typical. History of Black water Fever: Difd Shiortily after Admission to the Hospital.

Specimen through the courtesy of Col. H. C. Clarke, Canal Zone, Panama. This and Plate 14 show a complete proximal convolution with its glomerulus. At the very origin of the convolution dilatation of Bowman space, dissolution of the hasement membrane and destruction of the tubule wall are seen (A). There then follow irregularly alternating disruptive lesions and stretches of convolution which are relatively better preserved. Magnification $82 \times$. 
Plate 14. The Distal Half of the Convolution (See Plate 13)

This shows more damage and the terminal medullary portion is the seat of an almost continuous disintegration of the basement membrane and necrosis of the tubular epithelium. In areas of relatively* normal tubule, droplets of absorbed heme pigment are visible, some positive to the Berlin Blue reaction and some to benzidine. These stain black with iron haemotoxylin and therefore are obscured in the upper portions of the convolution by the mitochondrial elements; where the latter are less dense (A), the fine pattern of heme pigmentation can be seen. The dark granular material in the desquamated dead cells in the lumen of the tubule (B) was positive to the Berlin Blue reaction. Magnification $82 \times$.

Insert shows detail of heme pigment granules at $\mathrm{A}$ at a magnification of $200 \times$. 


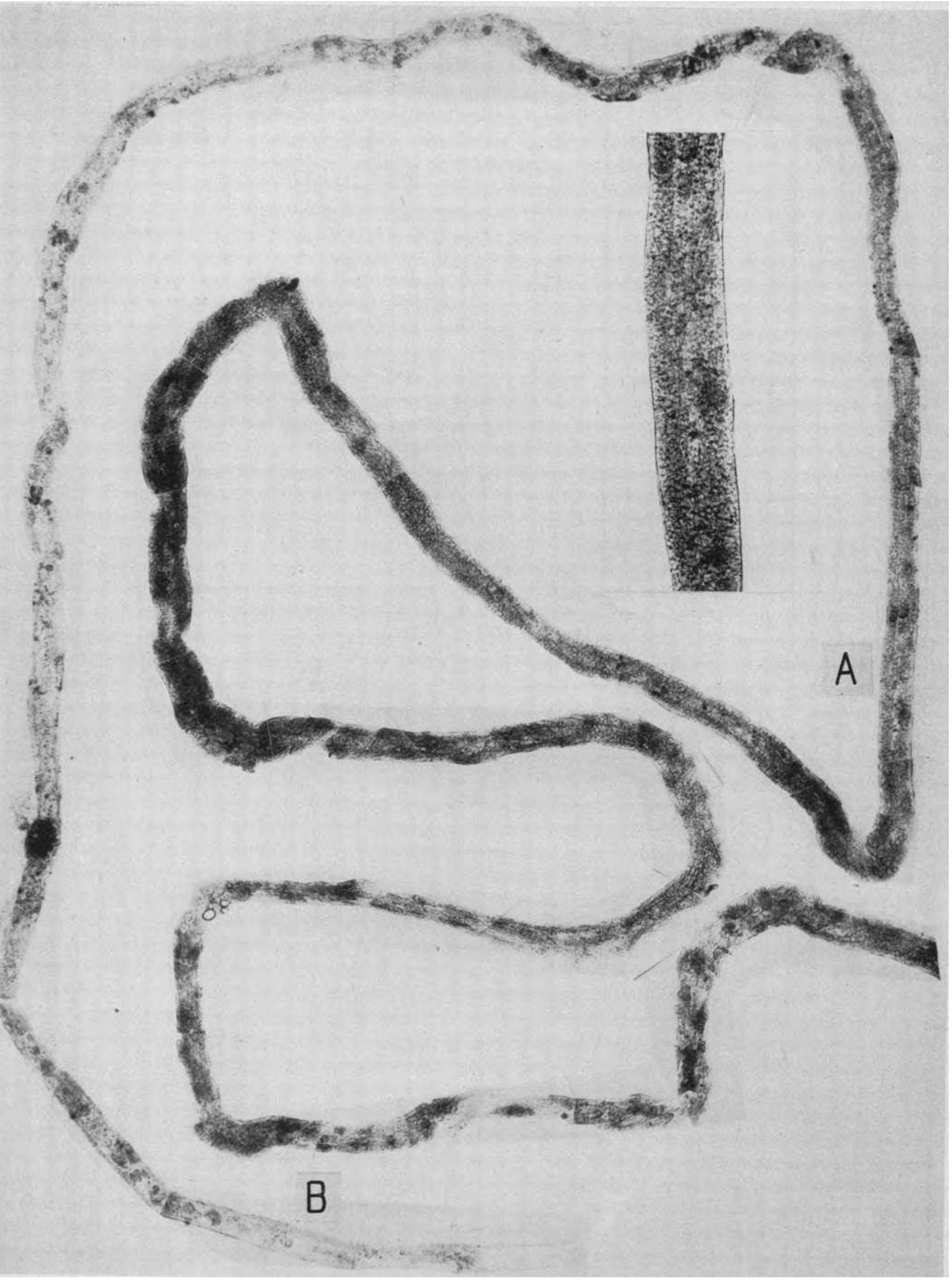




\section{Plate 15. Ascending Limb and Distal Convolution from}

the Same Kidney as in Plates 13 and 14

A dilated ascending limb on the left, lined by an atypical epithelium. Note the similarity of this abnormal distal portion of the nephron to the abnormal regenerated terminal portion of the proximal convolution in Plate 12, except that the ascending limb does not contain granules or droplets of absorbed heme pigments. A complete dissolution of basement membrane, necrosis of epithelium and tubular disruption in the distal convolution at $\mathrm{A}$. Scattered along the length of the entire specimen are collections of heme pigment impregnated debris. (B) As is usual, the tubule wall is less damaged at these points. Magnification $82 \times$. 
Plate 16. Slimate Poisoning;

Details of clinical history in text, page 1314. Died on the 29th day.

The terminal medullary portion of a proximal convolution. The cortical portion of this convolution showed the typical nephrotoxic necrosis due to mercury. This lesion is visible in the horizontal stretch of tubule above, $A$, an intact basement membrane outlining the dead mass of dark stained necrotic debris. At $B$ there begins a long segment of tubule, greatly swollen, in which the basement membrane has completely disintegrated and the tubule wall is destroyed. The tubular disruption ends abruptly (C) and the convolution continues, its basement membrane intact, to the passage into the thin loop of Henle's loop. At the point of greatest disruption $\left(\mathrm{B}^{1}\right)$ irregular regeneration of the epithelium is evident in the clusters and masses of cells with large vesicular nuclei. It is also evident that this regeneration has not repaired the tubule wall or reestablished a lumen; this, apparently due to lack of a basement membrane to direct the course of the proliferating cells. Magnification $82 \times$.

Insert $\mathrm{B}^{1}$ shows the disruptive lesion at $\mathrm{B}^{\mathrm{x}}$ at a magnification of $200 \times$. For the appearance in histological section of a similar lesion $c f$. Figure 1.

Insert A shows a typical nephrotoxic tubular lesion in the terminal segment of a proximal convolution of a nephron from the kidney of a dog which had received an intravenous injection of $75 \mathrm{mg}$. of corrosive sublimate. The renal epithelium is completely necrotic and reduced to granular debris. Note the intact basement membrane, at places out of focus and therefore invisible, which maintains the tubular contours. Magnification $200 \times$. 
Plate 17. Two Distal Convolutions and the Origin of the

Collecting Tubule from the Same Kidney as in Plate 16

At the lower right a distal convolution is filled in great part with dark staining casts which in histological section stained with eosine and contained no heme pigment. The second half of the upper distal convolution is similarly filled and the cast extends some distance into the collecting tubule on the extreme left. The original thick epithelium of the first portion of the upper distal conyolution has been replaced by a flat atypical regenerated layer of cells which has not completely healed the disruption that had occurred in the tubule wall (A). Magnification $83 \times$. 


\section{Plate 18. Potassium Chilorate Potsoning}

Details of clinical history in text, page 1316. Died on the 11th day.

A complete proximal convolution. The dark staining poorly preserved mitochondria in the first half of the convolution indicates a relative integrity of the tubular epithelium, although there may be some lesser form of damage such as "cloudy swelling." Beginning at the arrow there is a frank necrosis of the tubular epithelium with desquamation which continues to the termination of the convolution in the narrow limb of Henle's loop. Magnification $84 \times$.

For the appearance in histological cross section of the partially necrotic upper half of a similar convolution, cf. Figure 2. 


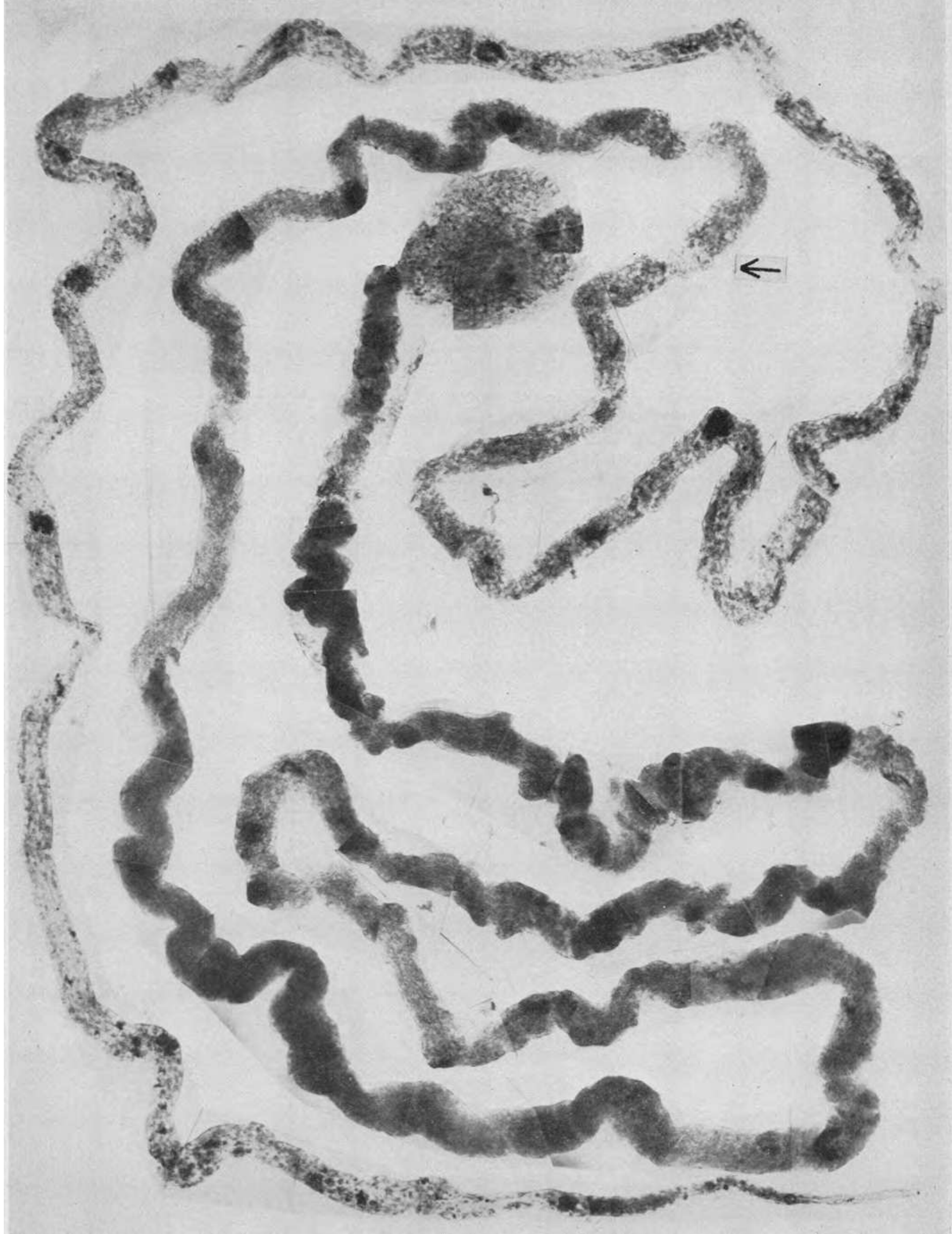


Plate 19. An Ascending Limb, Below, and Distal Convolution from the Same Case as in Plate 18

The ascending limb is dilated but its cellular pattern is fairly well preserved. At $\mathrm{A}$ is a huge cast which blocks the distal convolution and proximal to it a greatly dilated segment with complete disintegration of the basement membrane and disruption of the tubule wall. Note the abortive regeneration of sheets of cells that grow at random. Magnification $84 \times$. 


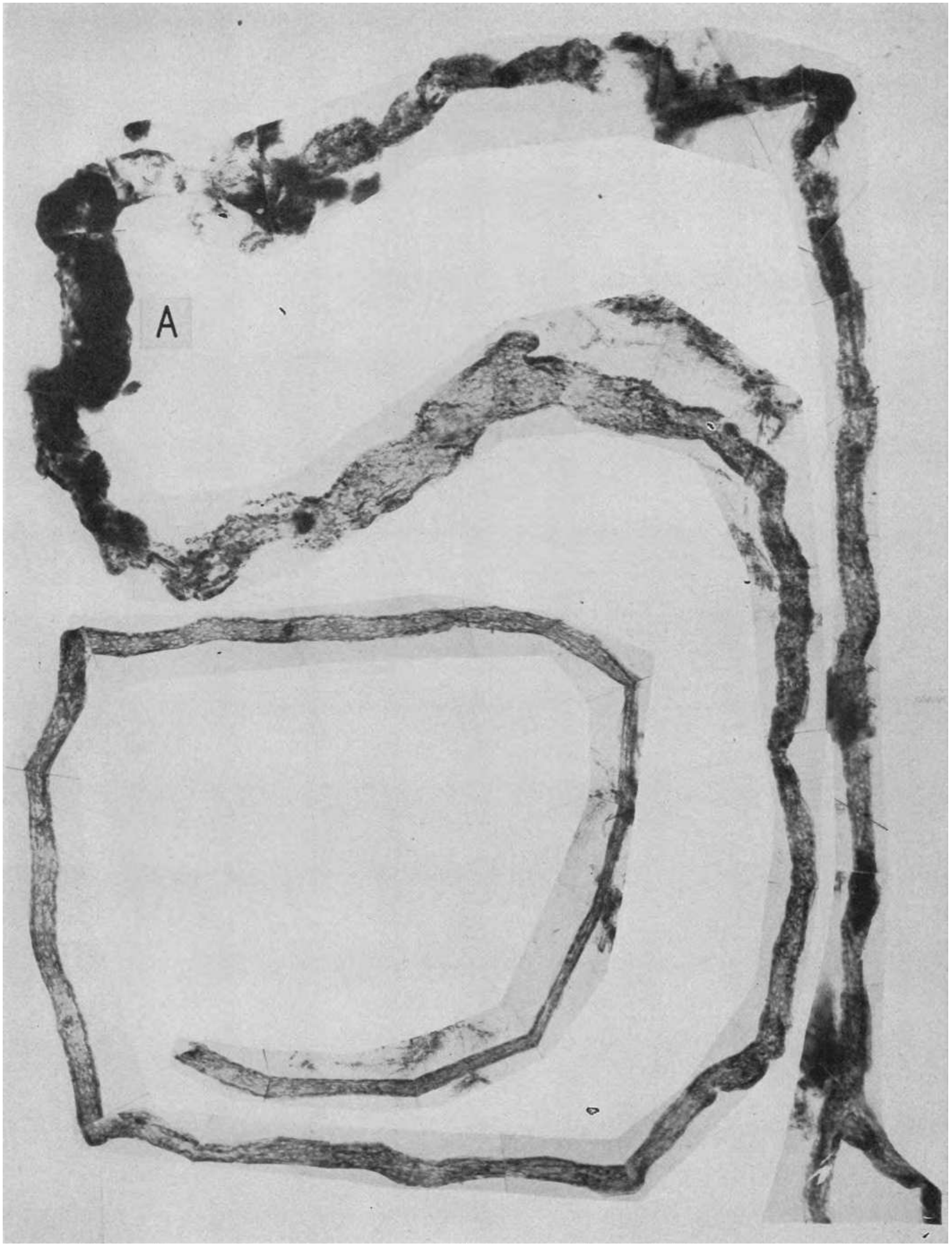




\section{Plate 20. Sulfonamide Intoxication}

Woman with sinusitis and a previous history of albuminuria took $3.4 \mathrm{gm}$. sulfathiazole in one day at home, and $3 \mathrm{gm}$. after entering hospital for severe headache and pain in back. Did not void in 12 hours, erythematous rash appeared, $60 \mathrm{cc}$. urine by catheter. Died 20 hours after admission (cf. J.A.M.A., 1942, 119, 8, Case 4). Specimen through the courtesy of Dr. Max Lederer.

A complete proximal convolution and its glomerulus. As was noted by Dr. Lederer in histological section there is necrosis of the renal epithelium throughout almost the complete length of the convolution. The basement membrane, however, is intact and its tube is stuffed with cellular debris in various degrees of consolidation. This case showed in extreme degree damage of the proximal convolution: as noted in our text there is great variation in the type of reaction of the kidney to the deleterious effects of the sulfonamides and many do not show the nephrotoxic effect on the proximal convolution that is well illustrated in this case. Magnification $80 \times$. 


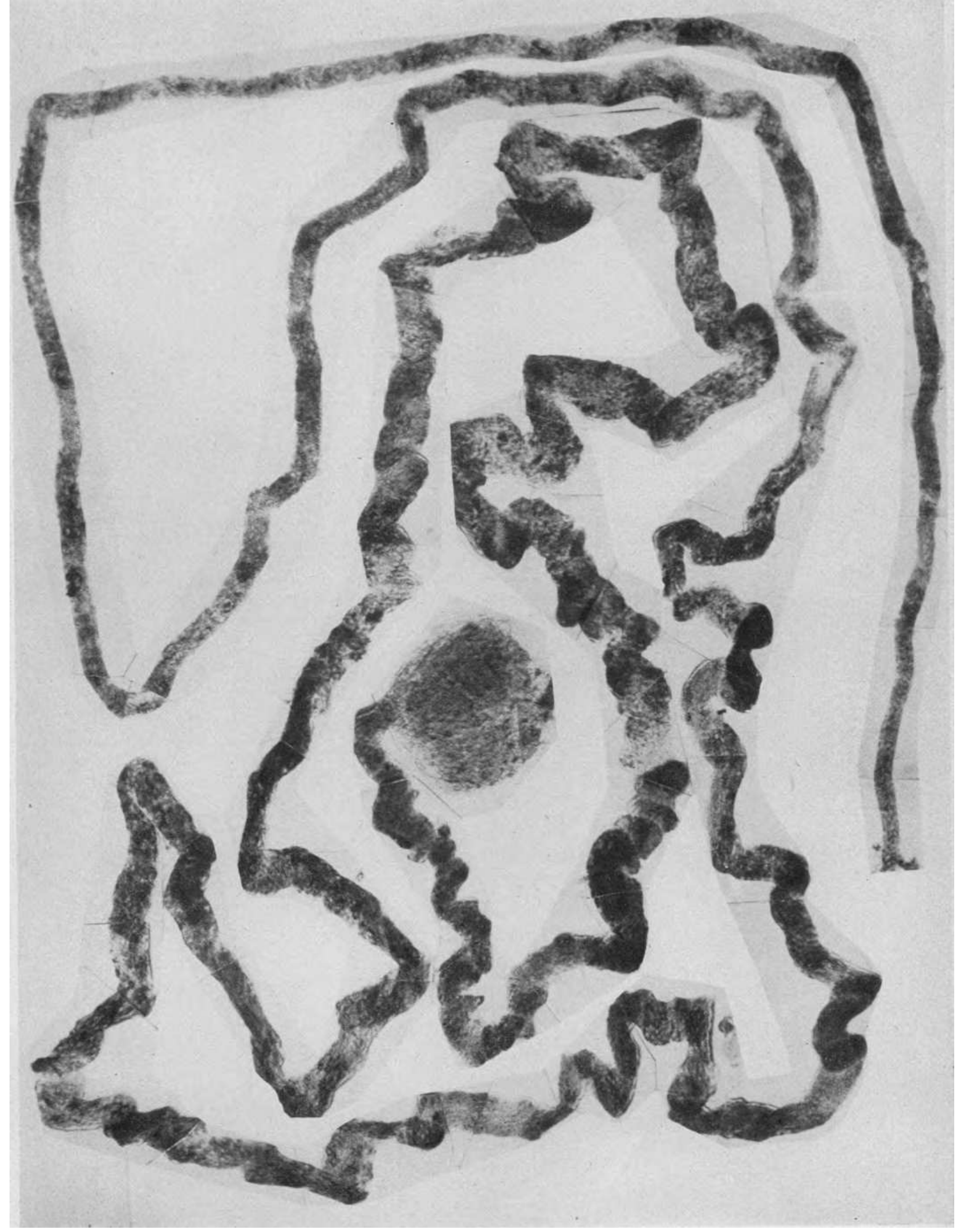


Plate 21. Sulfonamide Damage-Male Admitted with a "Cold"

Symptomless treatment with sulfathiazole one month previously. Given 13 gm. sulfathiazole in 48 hours; nausea, vomiting, urinary suppression, $150 \mathrm{cc}$. in four days, and death in uremia eight days later. Specimen through the courtesy of Dr. Baldwin Lucké.

Ascending limb and two distal convolutions and collecting tubule. The ascending limb (left) shows an intact epithelium. At $A$ and $A^{1}$ the distal convolution shows the disruptive lesion; at $B$ there is a cast distending the intact tubule. At A a projecting sheet of atypical epithelial cells can be seen but the disruption is not filled. The other distal convolution, above and to the right, shows less damage; at $\mathrm{C}$ there is an incipient solution of continuity of the basement membrane. In the collecting tubule, below, the typical epithelial pattern is well preserved; the lumen contains masses of debris and desquamated epithelial cells. Magnification $83 \times$.

Insert shows detail of disruptive lesion at $A$ at a magnification of $200 \times$. 


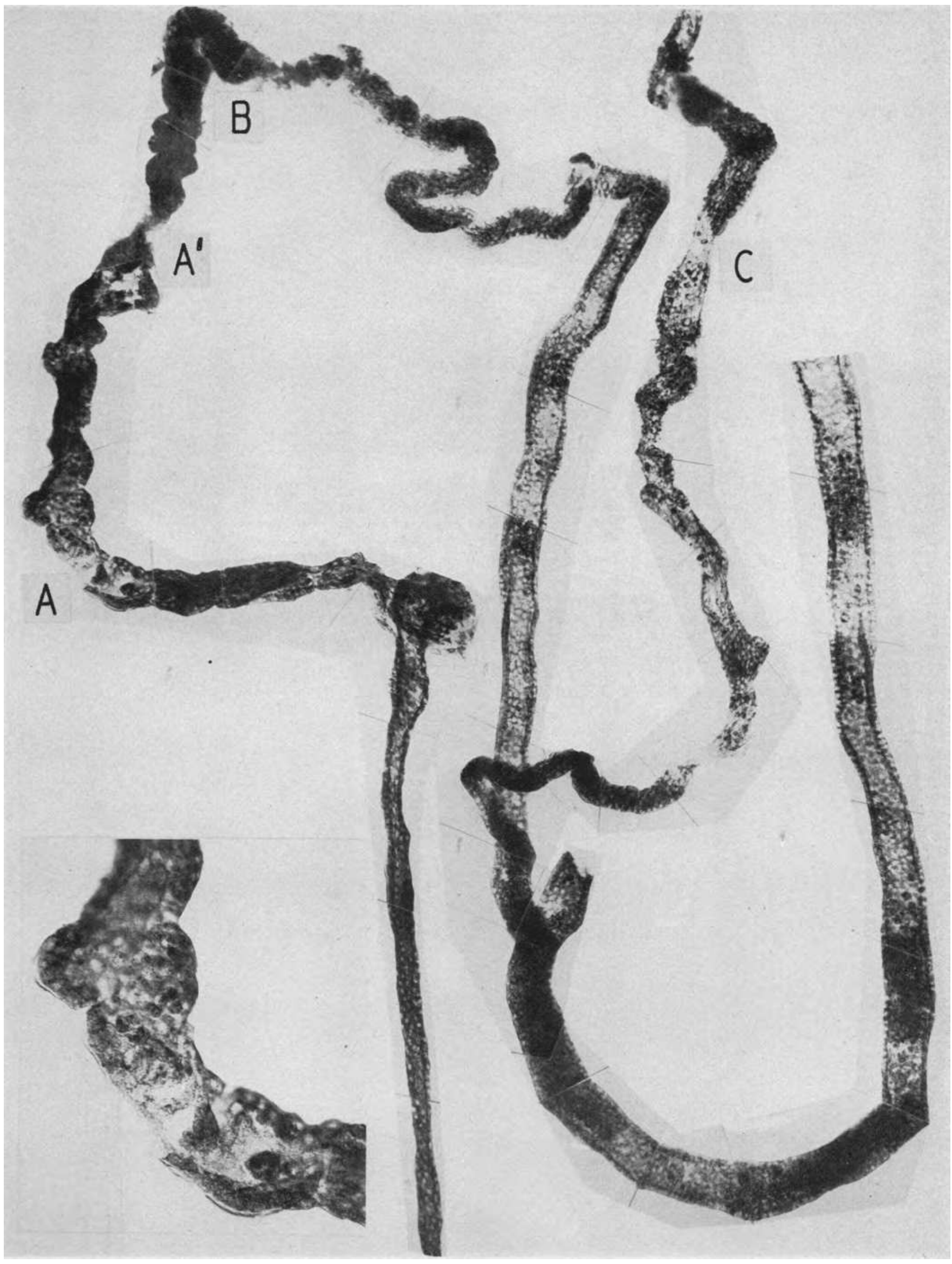


Plate 22. Di-Ethylene Glycol. Poisoning

Details of history in text, page 1322. Died on the 12th day. Specimen through the courtesy of Dr. Milton Helpern.

A portion of the proximal convolution. The dissection of proximal convolutions from this kidney was exceedingly difficult because there was a combination of a nephrotoxic vacuolar necrosis of the epithelium and a disruption of the basement membrane (A) throughout the convolution. For the appearance in histological section of the lesion see Figure 3. The specimen was under-differentiated for purposes of contrast as the almost complete vacuolization of the renal epithelium left little to stain by the usual procedure. For the typical appearance of a lesser and limited lesion of di-ethylene glycol under experimental conditions, see Plate 44. Magnification $84 \times$. 


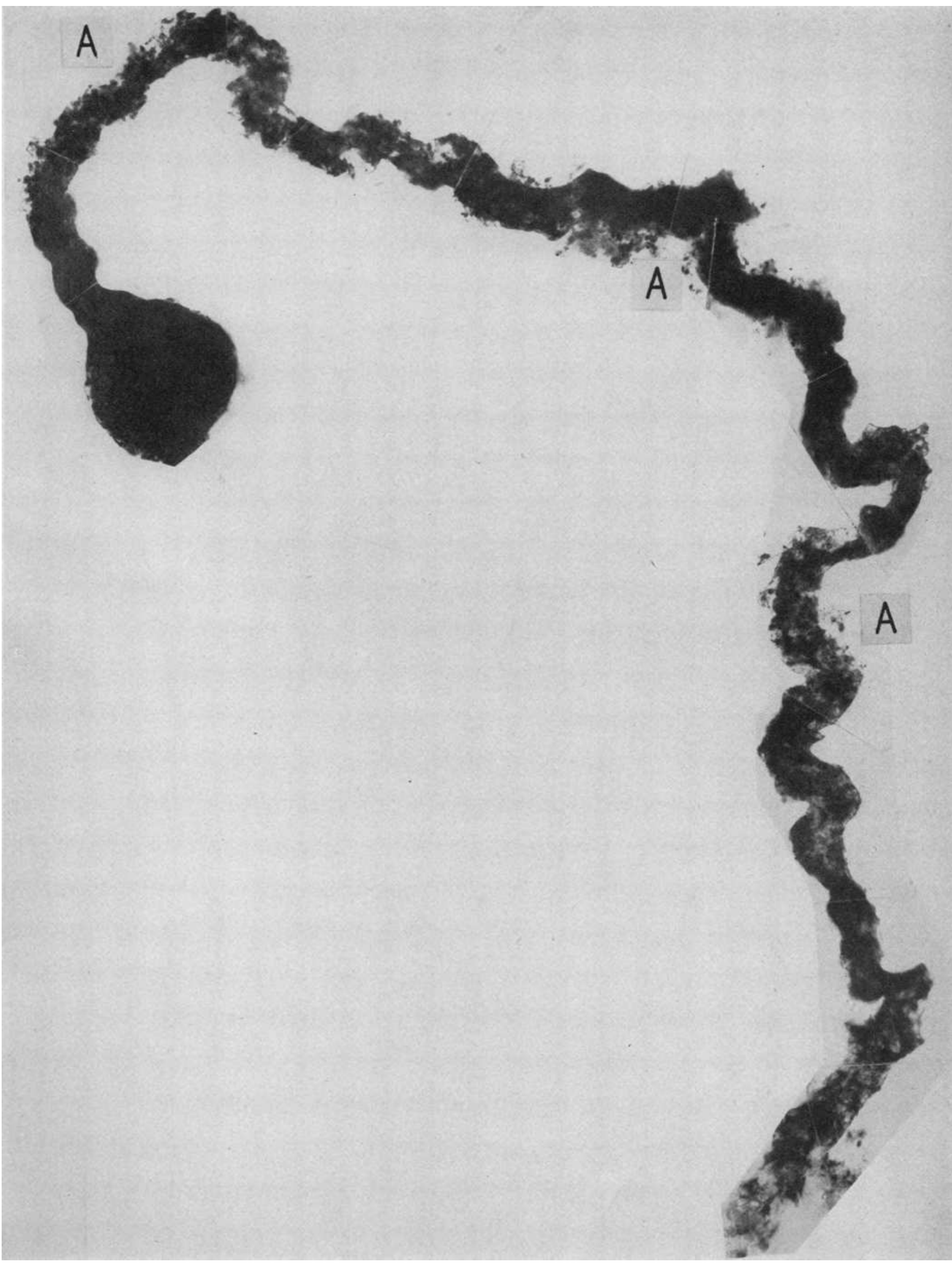


Plate 23. Ascending Limb with Distal Convolution on the Right Joining Another to Form a Collecting Tubule; from the

Same Case as ix Plate 22

There are casts of debris in the ascending limb and in other portions of the various tubules at $A$. At $B$ the distal convolution is greatly swollen, the basement membrane disintegrated and the tubule disrupted. The distal convolution on the left is fairly well preserved; the wall of the collecting tubule is intact and its lumen contains debris and a consolidated cast. Magnification $80 \times$.

Insert shows detail of disruptive lesion at $B$ at a magnification of $200 \times$. 


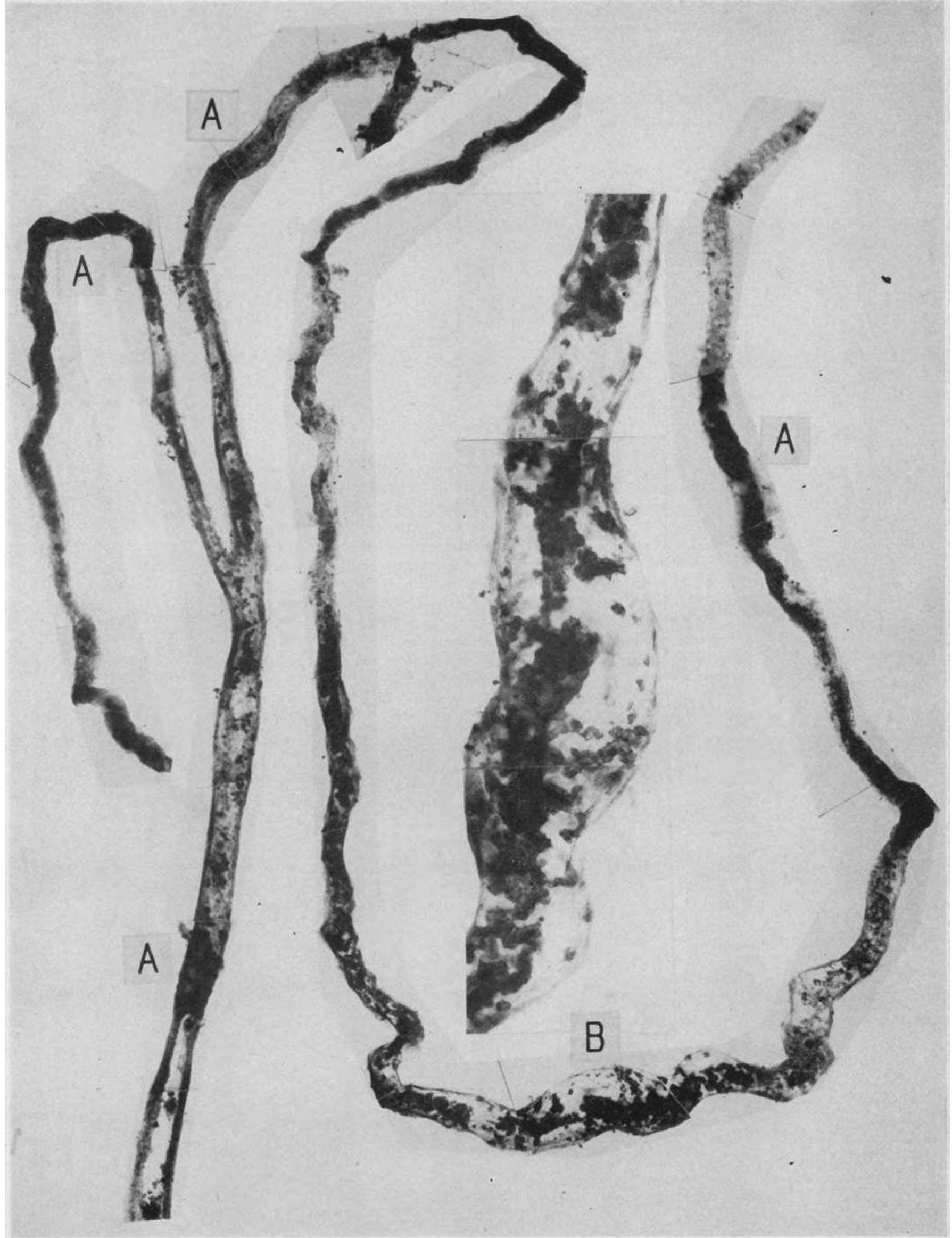


Plate 24. Di-Ethylene Glycol Poisoning-Kidney of the Other Individual. Who Was Poisoned at the Same Time bl: Liven 24 Days

(See text, page 1322 , for detailed history.)

Glomerulus and first half of proximal convolution. In contrast with the intensely damaged convolution shown in Plate 22, extensive regeneration of the tubular epithelium has occurred in the 12 days following the biopsy. See Figures 4 and 5 for the change that occurred in this time. The dissected specimen shows, however, the inadequacy of this marked epithelial proliferation so far as a restitution of a functioning nephron is concerned. Masses and sheets of proliferating renal epithelial cells impinge upon and occlude the lumen with the production of valve-like flaps and whorls of cells (C). As a result, irregular constrictions (A) alternate with pouch-like protrusions or diverticula (B). At many places the earlier disruption of the tubular wall persists (D) though one "side" of the tubule wall seems repaired. The contrast of the real inadequacy of this repair, as seen in the dissected nephron with what appears to be good "tubular regeneration" in the histological section (Figure 5), is noteworthy. Magnification $82 \times$. 


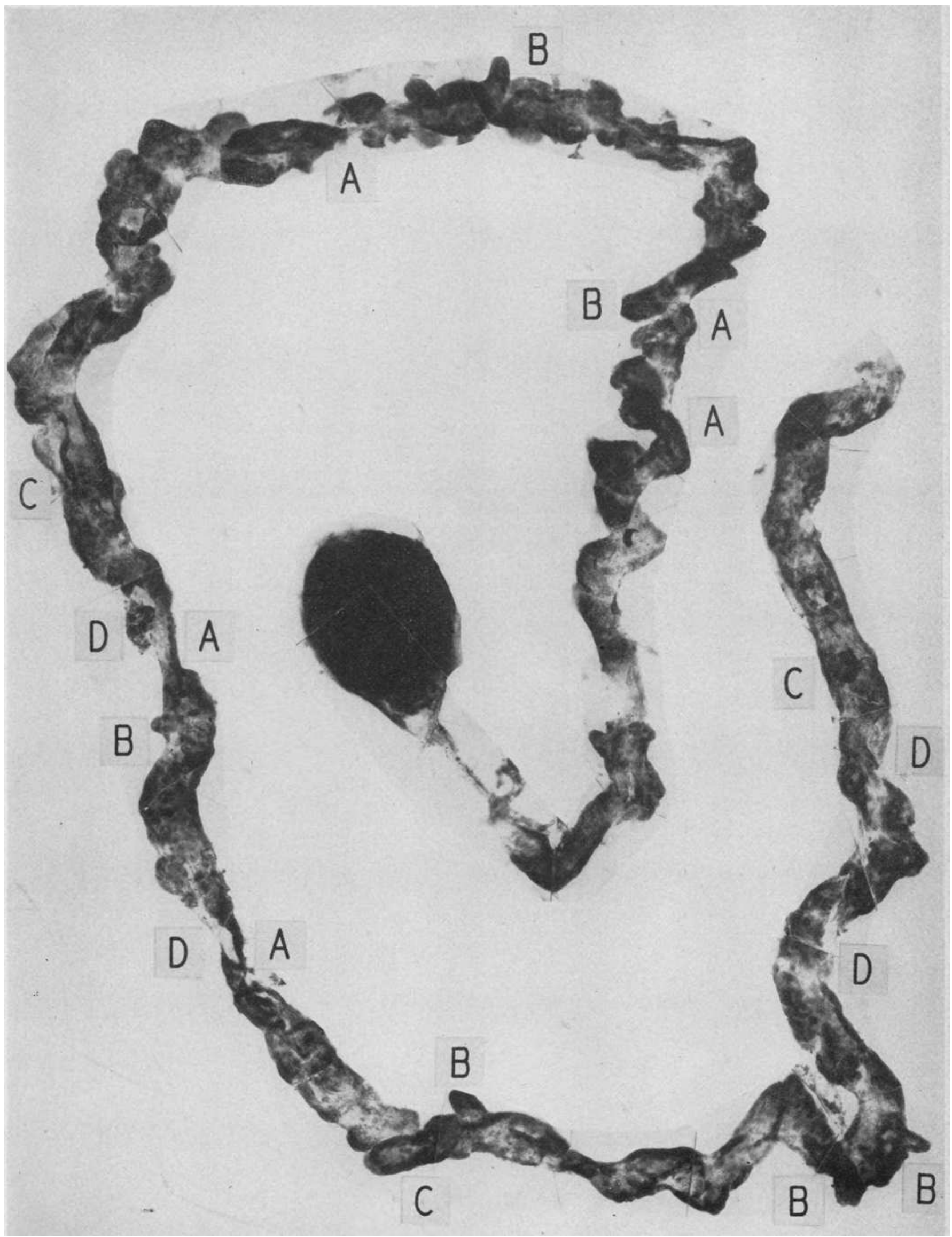




\section{Plate 25. Ascending Limb, Distal Convolltions and Coliecting}

Tubule from the Same Kinney as in Plate 24

Extensive casts (A) are seen in various portions of the tubules; at $B$ and $\mathrm{B}^{1}$ there are disruptions of the distal convolution, the latter surrounded by interstitial reaction and large capillaries. There is extensive regeneration in the ascending limb and distal convolution on the right. From $\mathrm{B}^{1}$ to $\mathrm{C}$ the detail of this incomplete repair can be seen in the irregular sheets of cells that imperfectly reconstitute the tubule wall. Magnification $80 \times$.

Insert shows the detail of the incomplete regeneration in the upper right distal convolution at a magnification of $200 \times$. 


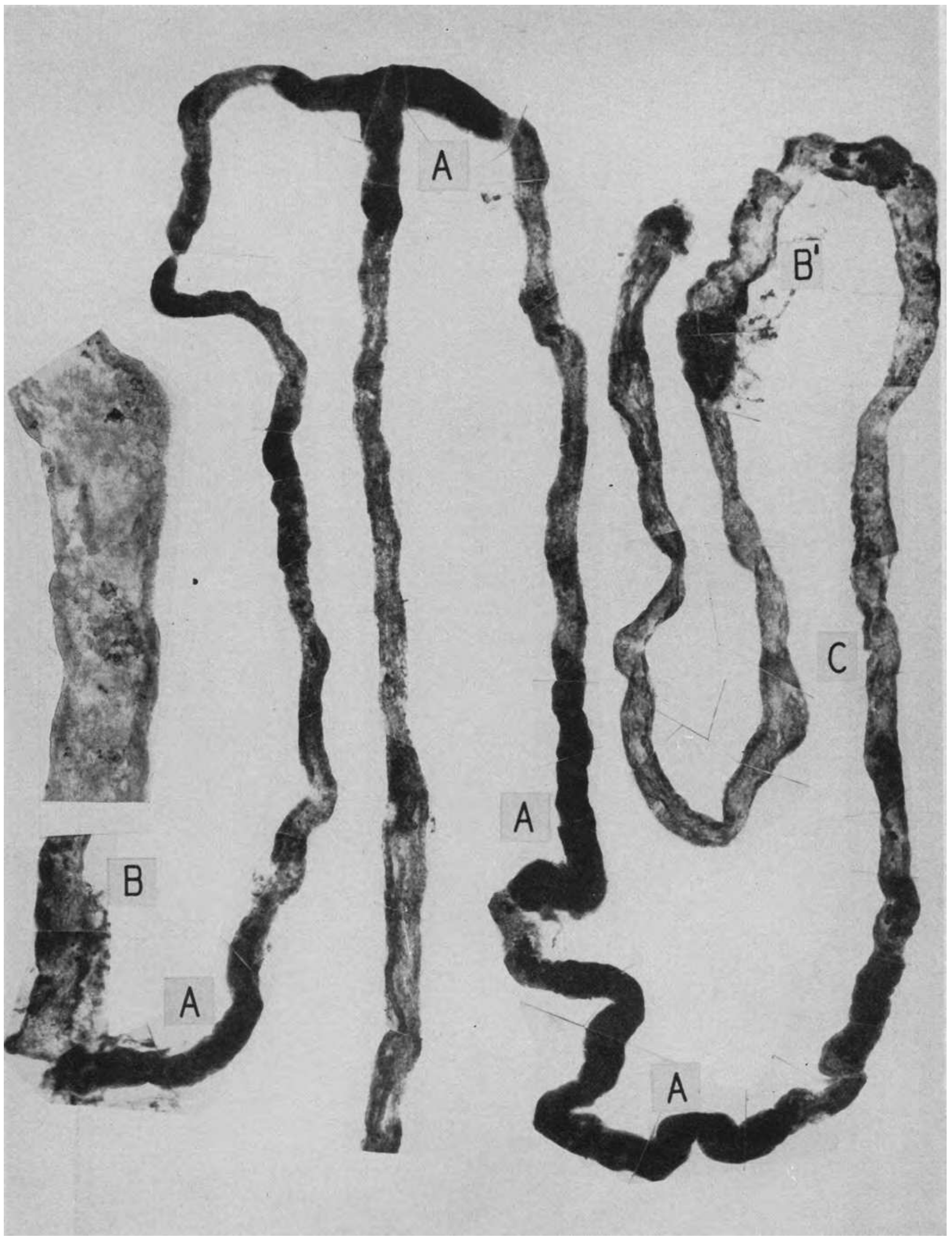




\section{Plate 26. Paroxysmal Hemoglobinutia}

Repeated attacks of cold induced hemoglobinuria; death 12 days after a particularly severe attack. (See details in text, page 1326.) Specimen through the courtesy of Drs. Alfred Plaut and R. Sussman.

Proximal convolution and its glomerulus. This nephron came from the periphery of a fibrous scar in the kidney which, since there were no vascular lesions to account for it, was apparently the result of the damage of an earlier attack. There is an irregular atrophy and distortion of the convolution; at $\mathrm{A}$ there are frank disruptive lesions with peritubular adhesions. Magnification $84 \times$. 


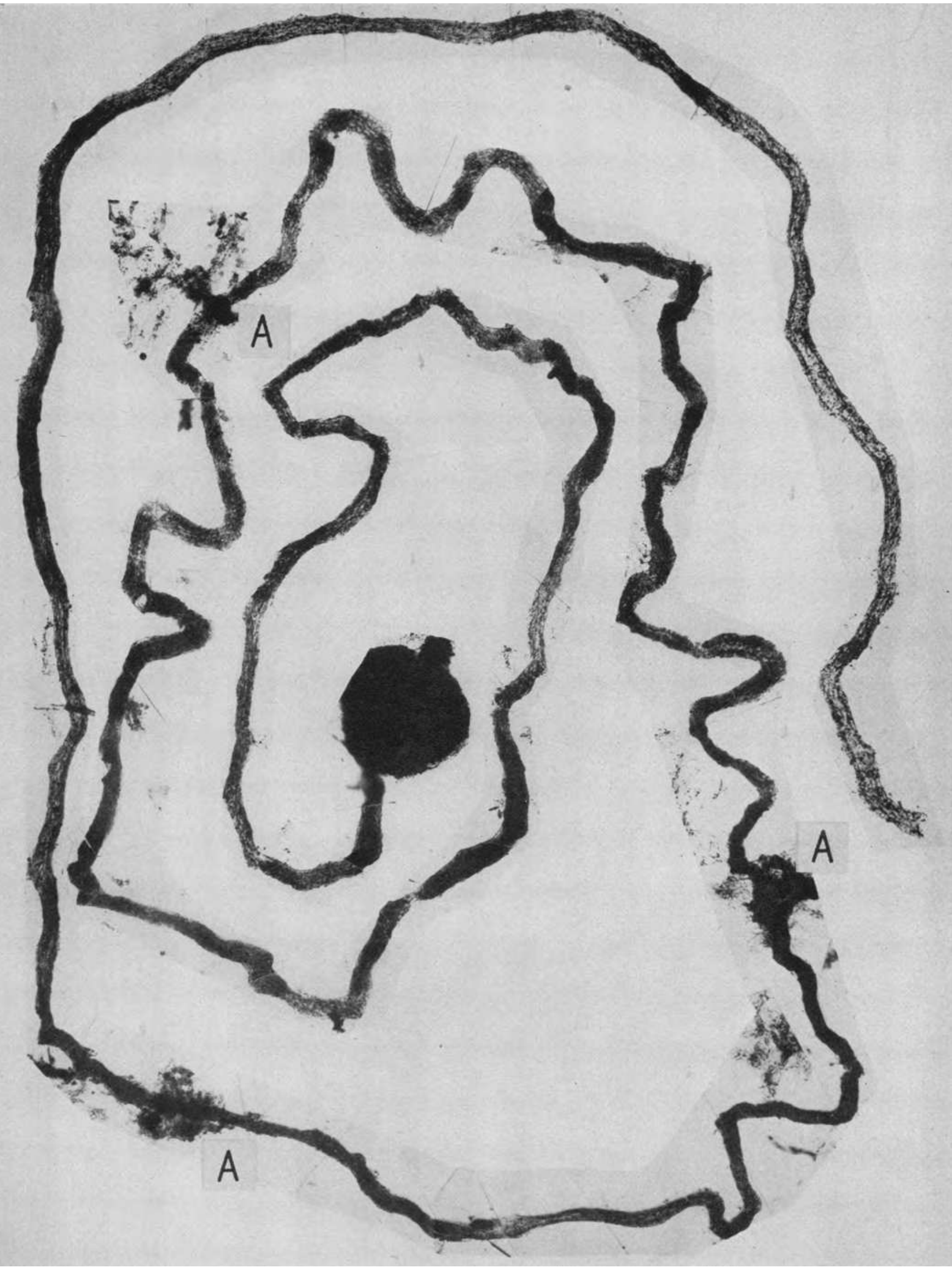


Plate 27. Ascending Limb and Distal Convolution from a Similar Fibrous Area of the Same Kidney as in Plate 26

Descending from the upper right, an ascending limb. It is difficult to decide in this atrophied and distorted nephron where the distal convolution begins, but since we have never seen diverticula elsewhere than on the distal convolution, presumably its origin is at A. There is a typical recent disruptive lesion at $\mathrm{B}$ with a small heme pigment cast in situ. These lesions were noted by Dr. Plaut in histological section and the similarity of the lesion to "crush injury nephrosis" noted. At $\mathrm{C}$ is a cystic dilatation of a segment of the convolution which contains some debris. Its angular irregular configuration strongly suggests the deforming effect of shrinkage of the connective tissue which surrounds it. Magnification $80 \times$.

Insert shows three large diverticula on another distal convolution which have the general configuration of glomeruli. Magnification $200 \times$. 


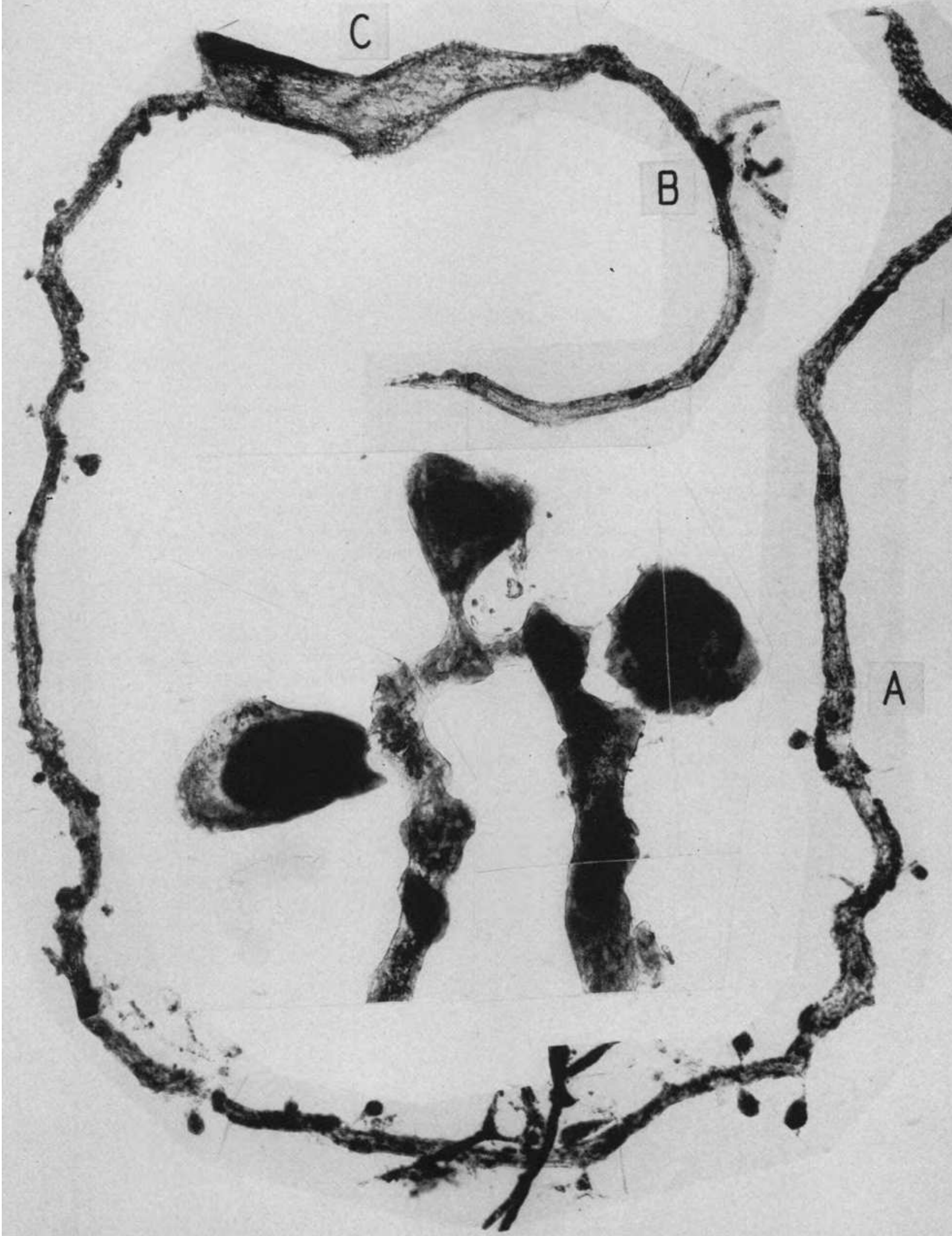


Plate 28. Dog K29

Under nembutal anesthesia the left renal artery was clamped for $66 \mathrm{~min}$ utes. Seventy-two minutes after removal of clamp a $350 \mathrm{cc}$. infusion was given, consisting of $50 \mathrm{cc}$. of the animal's red cells laked in distilled water with salt added to isotonicity and $50 \mathrm{cc}$. of the animal's plasma. The animal was killed five days later.

A complete proximal convolution with its glomerulus. The first part of the convolution shows a complete disintegration of its basement membrane and disruption of the tubule wall. The convolution from here on is remarkably well preserved; note perfect delineation of the rodlets in the basal portion of the renal cells in the insert. At $A$ this mitochondrial pattern is disturbed and at $\mathrm{B}$ the basement membrane is disintegrated and the cellular detail lost. This portion of the proximal convolution in the dog normally contains much visible fat and this is the source of some of the granular appearance noted in this region.

A most striking feature of the disruptive lesion due to the renal ischemia in all of the experiments, was the immediate continuity of stretches of completely damaged and perfectly preserved normal tubule; there was therefore no general toxic damage in these kidneys in spite of the infusions of haemoglobin. Magnification $82 \times$.

The insert shows the perfect preservation of the mitochondrial rodlets in areas of tubule which have escaped ischemic disruptive damage. Magnification $200 \times$. 


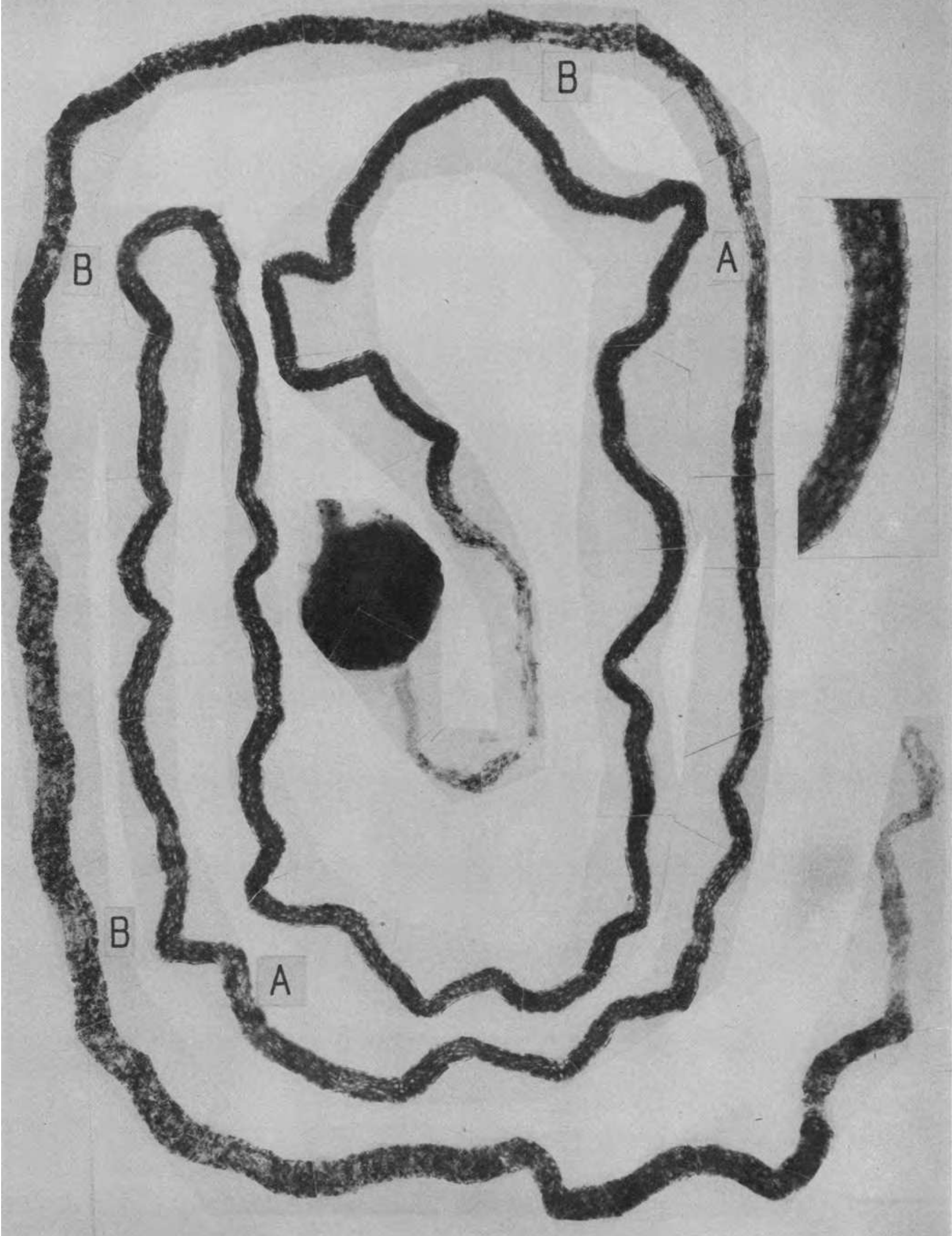


Plate 29. Ascending Limb and Distal Convolution from the Same Kidney as in Plate 28

The ascending limb on the left is well preserved. At $A$ the tubule is intact but distended with heme pigment casts that were positive to benzidine and negative for free iron by the Berlin Blue test. At $B$ and $\mathrm{B}^{1}$ there is disintegration of the basement membrane and a complete disruption of the tubule wall. At $\mathrm{B}^{1}$ crystals of heme pigment are present. Magnification $84 \times$. 


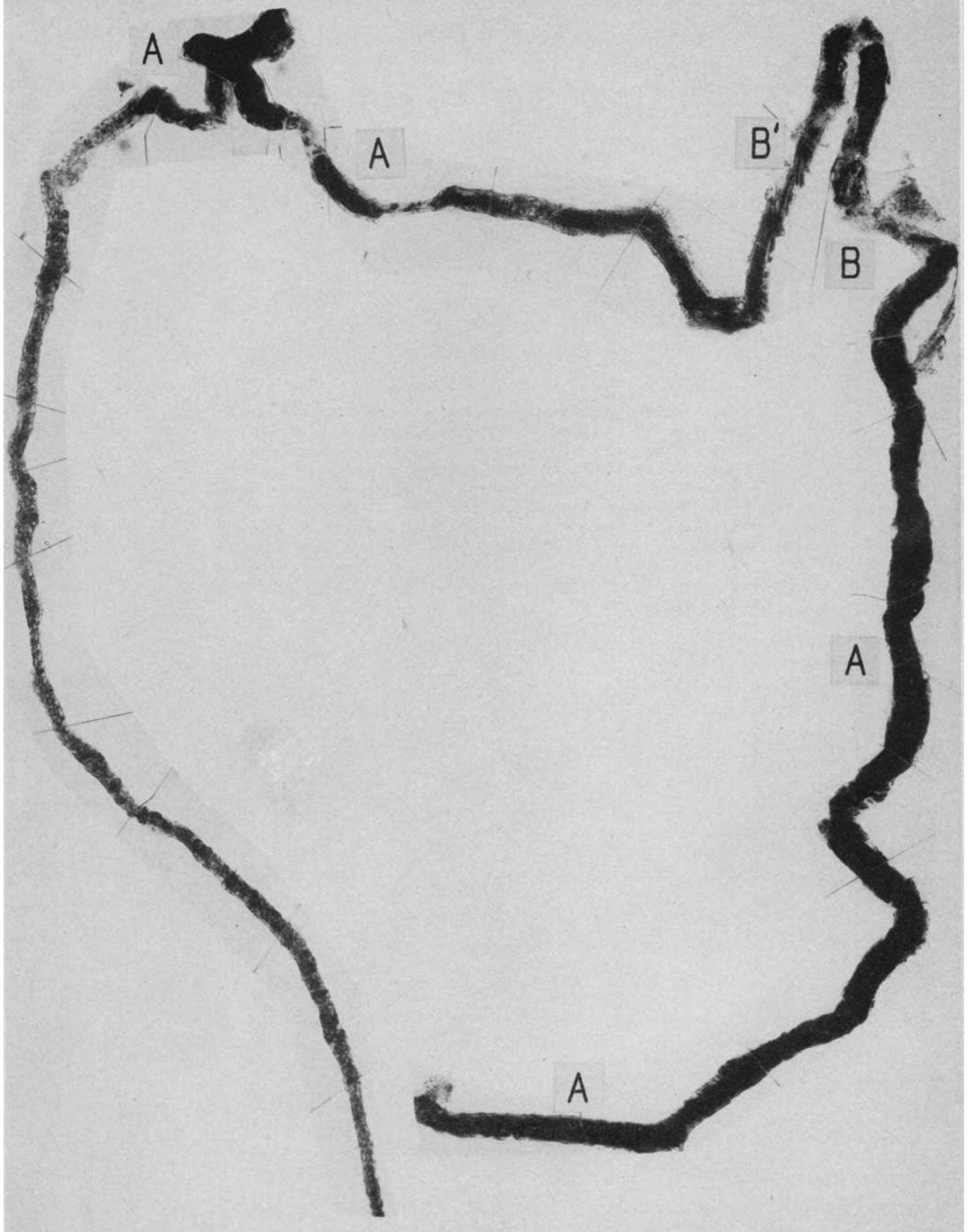




\section{Plate 30. Dog K26}

Under nembutal anesthesia the right kidney was removed and a serrefine placed on the left renal artery for 60 minutes. The animal remained in good clinical condition and was killed on the fourth day after the operation.

A proximal convolution. The greater part of the tubule is well preserved as is shown by the mitochondrial pattern; at $\mathrm{A}$ even the detail of the normal rodlets can be seen. At the arrows this pattern is disturbed and a disintegration of the basement membrane is evident. At B the lesion is fully developed, the tubule is swollen, the basement membrane disintegrated and the renal epithelium necrotic. At $C$ there is a collection of heme pigment impregnated debris; note the continuity and normality of the basement membrane. Magnification $80 \times$. 


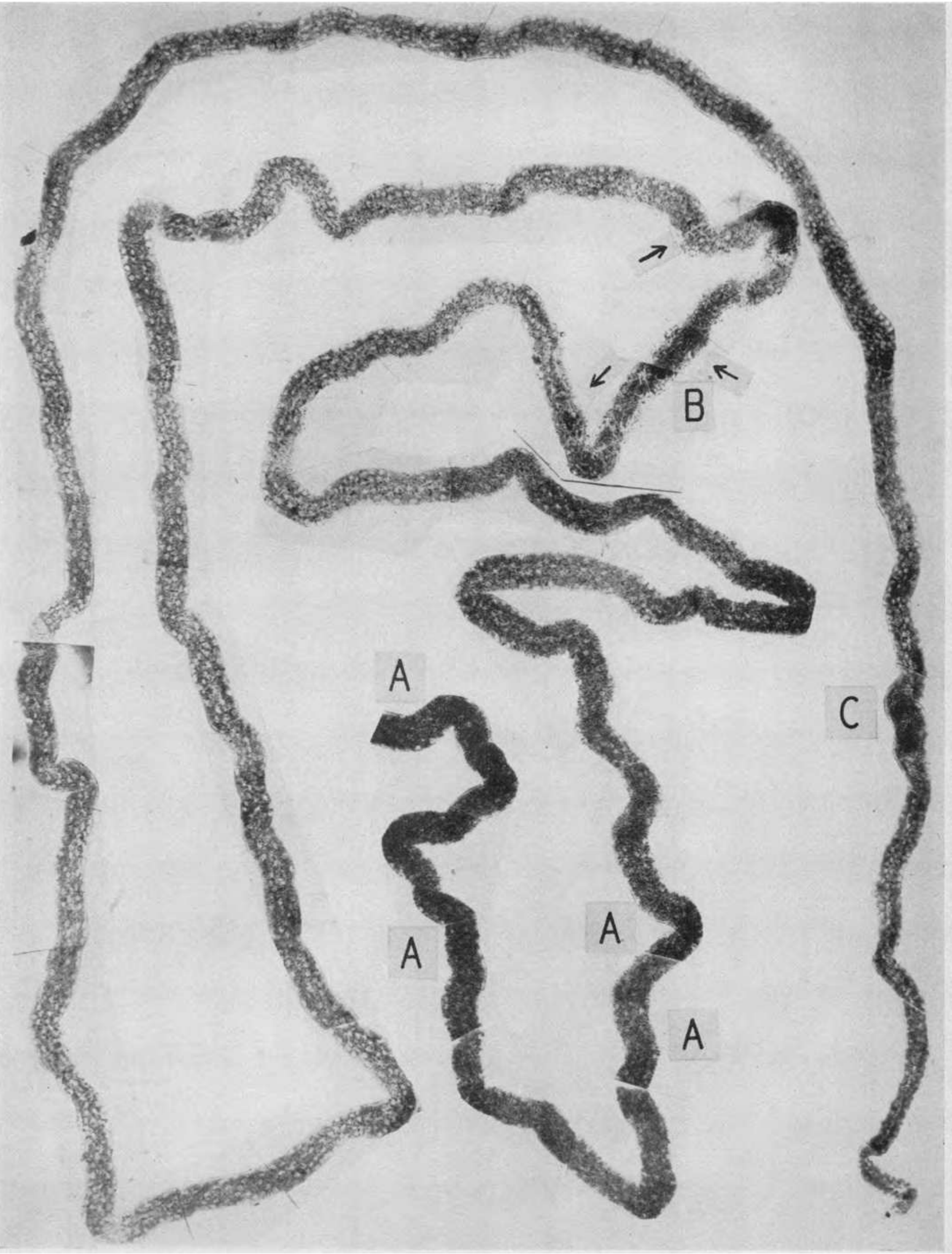


Plate 31. Three Distal Convolutions from the Same Kidney (Plate 30) Join to Form a Collecting Tubule on the Left

At $\mathrm{A}$ are seen the infrequently mentioned intercalated cells (12) common to all species in this portion of the renal tubule. All of the tubules are essentially normal except at $B$ where an incipient disruption can be seen. At $C$ the distal convolution contains a densely stained heme pigment cast; note that the tubule wall is not disturbed by its presence. Magnification $80 \times$.

Insert shows the detail of collecting tubule at $\mathrm{A}$ with granular intercalated cells. Magnification $200 \times$. 
Plate 32. Dog K33-The Results of Extreme Renal Ischemia of 200

Minutes Duration as Measured by Clearances Following Trauma

under Nembutal Anesthesia to Muscle Masses

A transfusion of the dog's own blood was given at the end of the experiment. Clinical condition of the animal was good on the fourth day. Blood urea 24.8. The animal was then killed.

Proximal convolution and glomerulus. Many nephrons from this kidney were structurally normal; others, perhaps $1 / 4$, resembled the illustration. The mitochondrial pattern of the greater part of the convolution is normal; from $B$ downward the dark staining is due to the normal presence of fat in the convolution. Compare with the similar segment of Plate 28 where both normal fat and disintegration of the basement membrane are present. At A and $\mathrm{A}^{1}$ are short segments of swollen convolution in which the basement membrane is fragmented, the renal cells necrotic and the tubule disrupted. These are shown at a higher magnification in Plate 33. At the arrows incipient lesions of the same sort are evident. Magnification $41 \times$. 


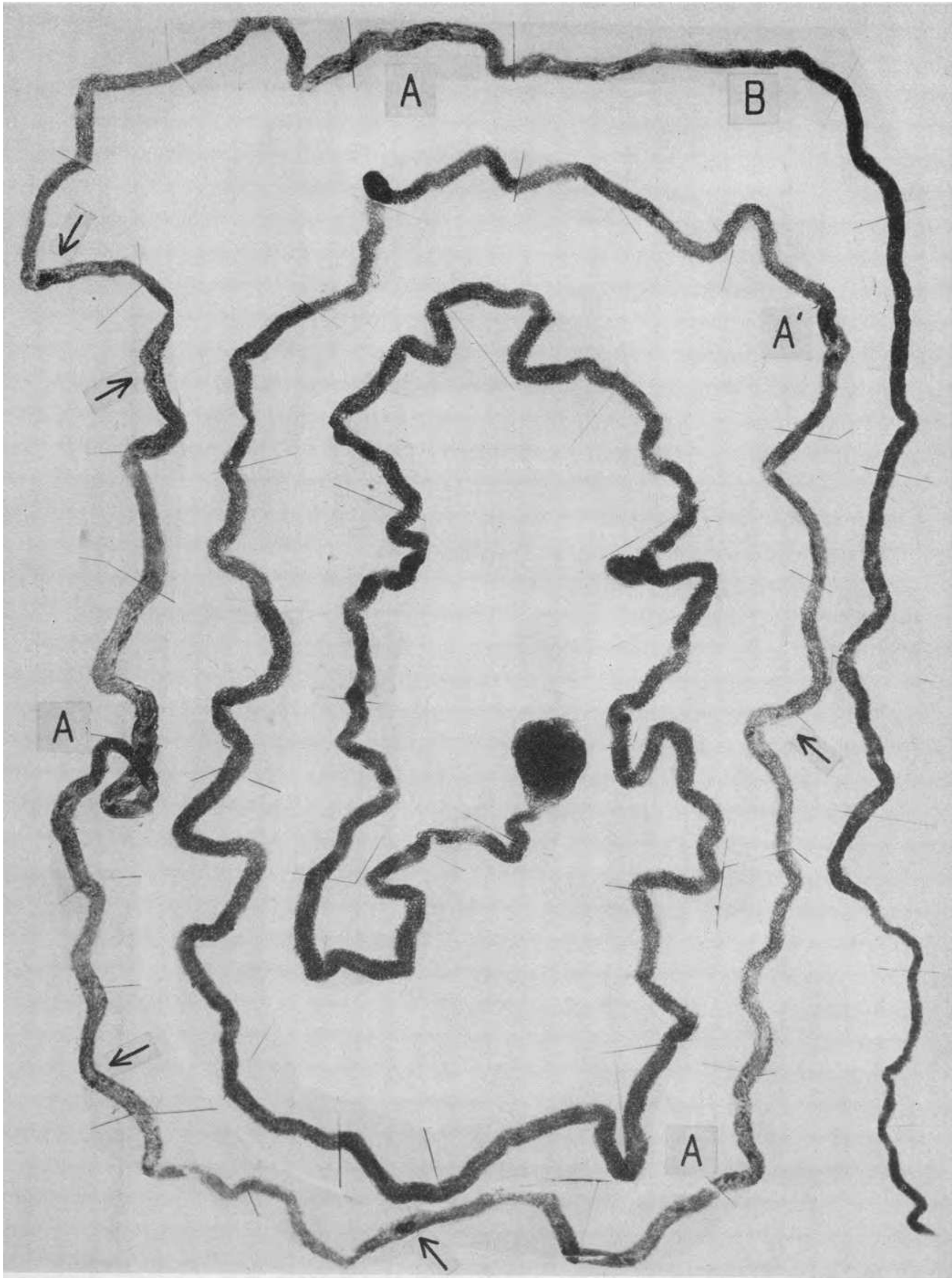


Plate 33. Ascending Limb axd Distal Convolution from tile Same Kidney as in Plate 32

Except for the presence of hene casts which have produced no tubular lesion the ascending limb is normal. Its upper portion and the greater part of the distal convolution are distended with heme casts; at $\mathrm{A}$ the presence of tubule wall disruption is evidenced by the surrounding adhesions of reactive intertubular tissue. Magnification $45 \times$.

Inserts show the detail of the disruptive lesions of the preceding plate at a magnification of $200 \times$. 


$$
\text { abs }
$$


Plate 34. Dog K32-Experimental Procedere as in the Experiment of Plates 32 and 33

A transfusion of the animal's blood was given at the end of the experiment. Before the experiment the blood urea was 10.9 ; on the fourth day 25.4 and on the seventh, 79.4. The animal, having convulsions, was killed.

Complete proximal convolution and glomerulus. The first $2 / 3$ of the convolution is fairly well preserved, though small areas are visible, as at $\mathrm{B}^{1}$, where the mitochondrial and nuclear pattern are disturbed as compared to the normality at $B$. Beginning in the terminal portion of the proximal convolution (A) there is an extensive disintegration of the basement membrane with epithelial necrosis and tubular disruption. Magnification $40 \%$. Lesser disruptions are evident at $A^{1}$ and $A^{2}$. For detail see following plate at a higher magnification. 


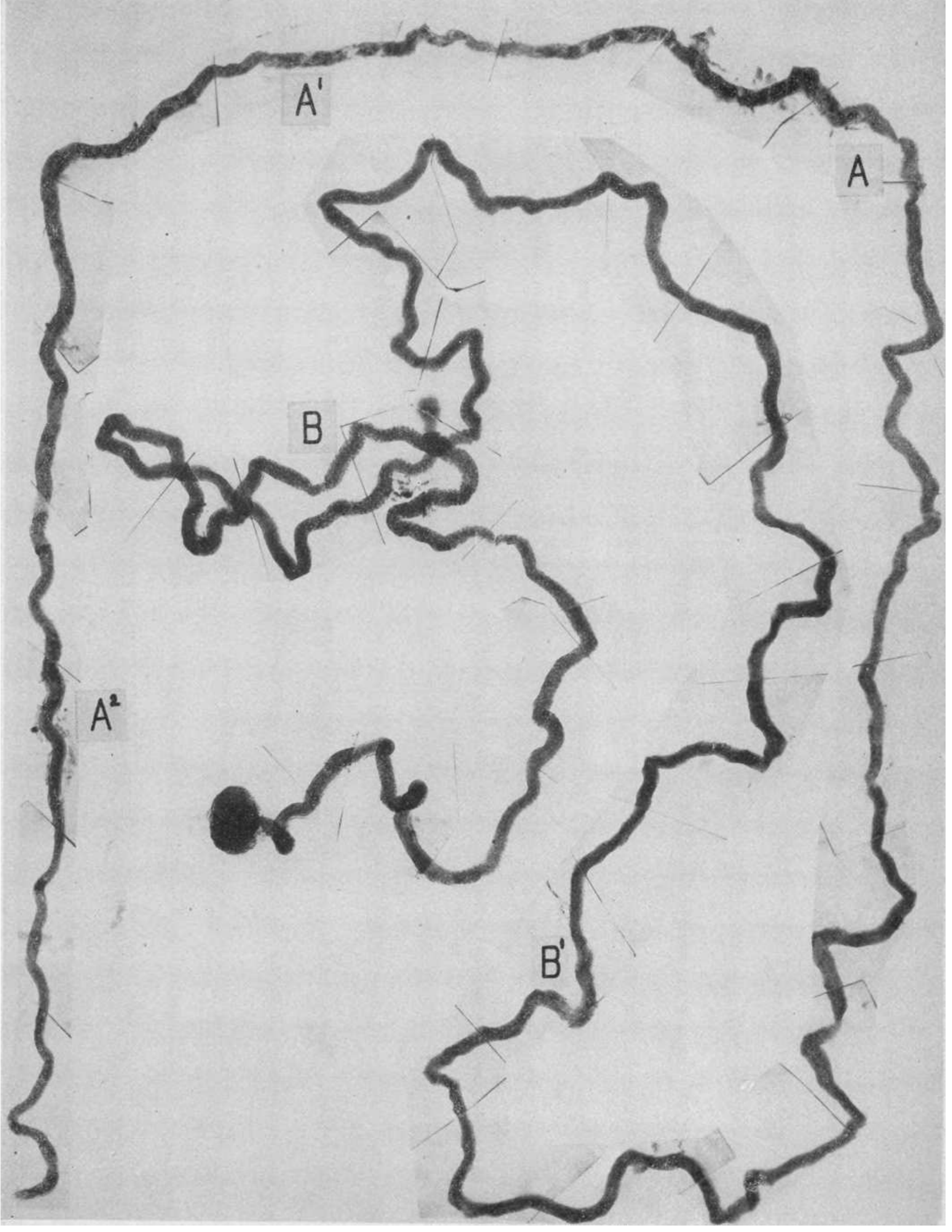




\section{Plate 35. A Higher Magnification of the Terminal Segment of}

Plate 34

The tubule begins in the center and it will be noted that its first part as it passes upwards is well preserved and the basement membrane intact. At A the epithelial cells are necrotic, the basement membrane disintegrated and the tubule disrupted; a short stretch of better preserved tubule with intact basement membrane follows and at $A^{1}$ another disruption. $A$ third disruption, less severe, is seen at $A^{2}$ and the tubule following to the thin limb of Henle's loop is again intact. Magnification $82 \times$. 


$$
\left\{3^{2}\right\}
$$




\section{Plate 36. An Ascending limb and Distal Convolution from the. Same Kinney as in Plate 34}

The ascending limb is entirely normal in its first portion; at $\mathrm{A}$ are heme casts. In the distal convolution at $B$ the tubule is swollen, the epithelium reduced to necrotic debris and its wall disrupted. Magnification $45 \times$.

Insert shows the disrupted distal convolution at $B$ at a magnification of $100 \times$. 


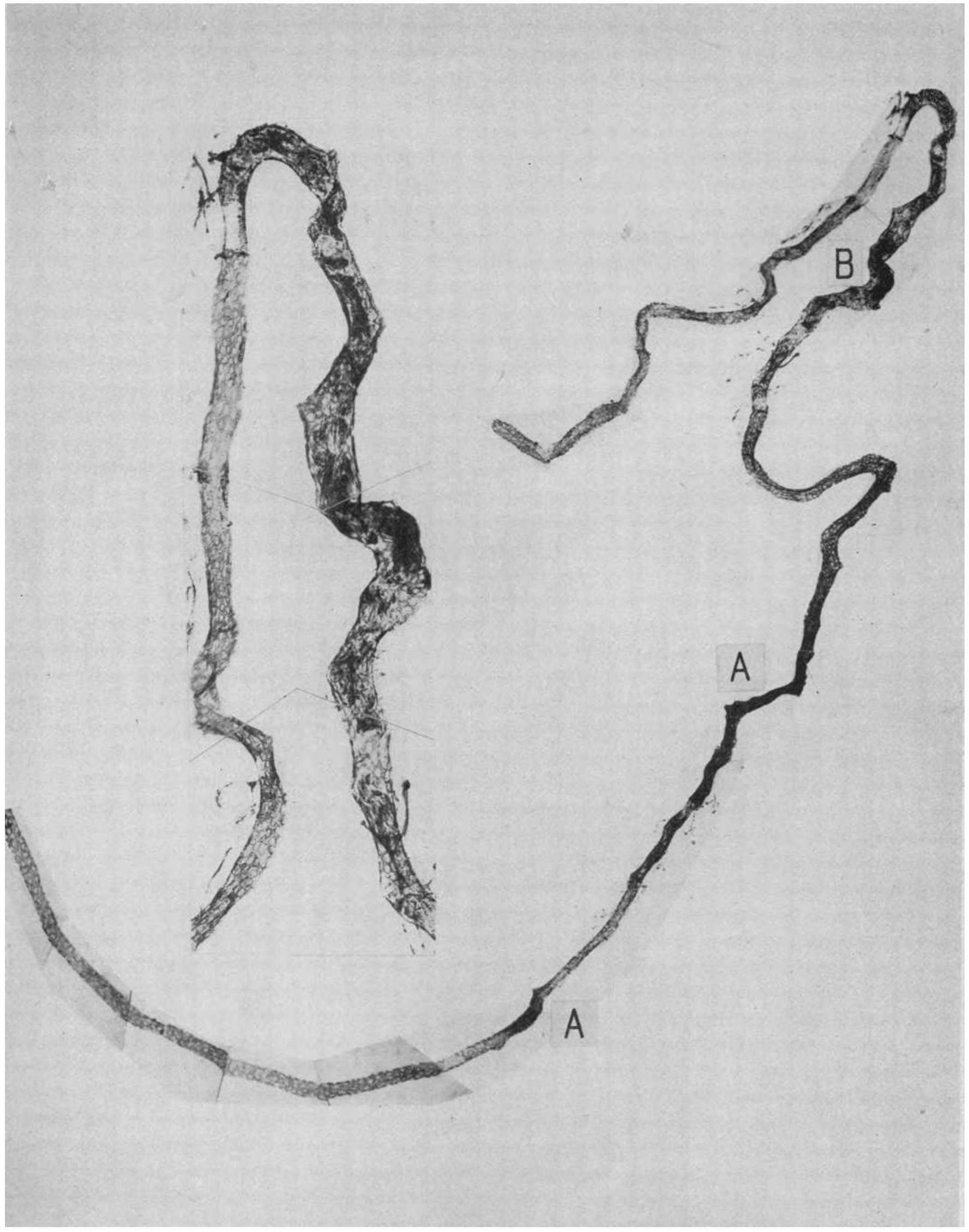


Plates 37 to 40

Circulatory Patterns in the Renal Cortex cxder Normal and Toxic Conditions as Shown by the Schlegel Technique

Magnification of all plates $16 \times$. The experimental animal received under anesthesia $1.0 \mathrm{cc}$. of $4 \mathrm{per}$ cent thioflavin $\mathrm{S}$ intravenously. The kidney was removed in six seconds, split, placed in glycerine and photographed under illumination by Wood's light. The fluorescent dye is held in the walls of the arteries and glomerular tufts. Capillaries show faintly and the veins not at all.

\section{Plate 37}

\section{A. Thie Cortical Circulation in a Normal Rabbit}

Note the even distribution of the blood to all glomeruli. The brilliant zone in the outer medulla is due to natural fluorescence of this tissue.

B. The Cortical Circulatory Pattern 19 Hours after the Subcutaneous Injection of 400 mg. of Sodium Potassium Tartrate

A large interlobar artery below with its arcuate branches. There is an irregular area of cortical ischemia where the dye in the blood did not reach glomeruli and intertubular capillaries. To the left an area of more nearly normal blood flow. The disruptive lesions in the nephrons which accompanied the irregular ischemia are seen in Plate 42. 


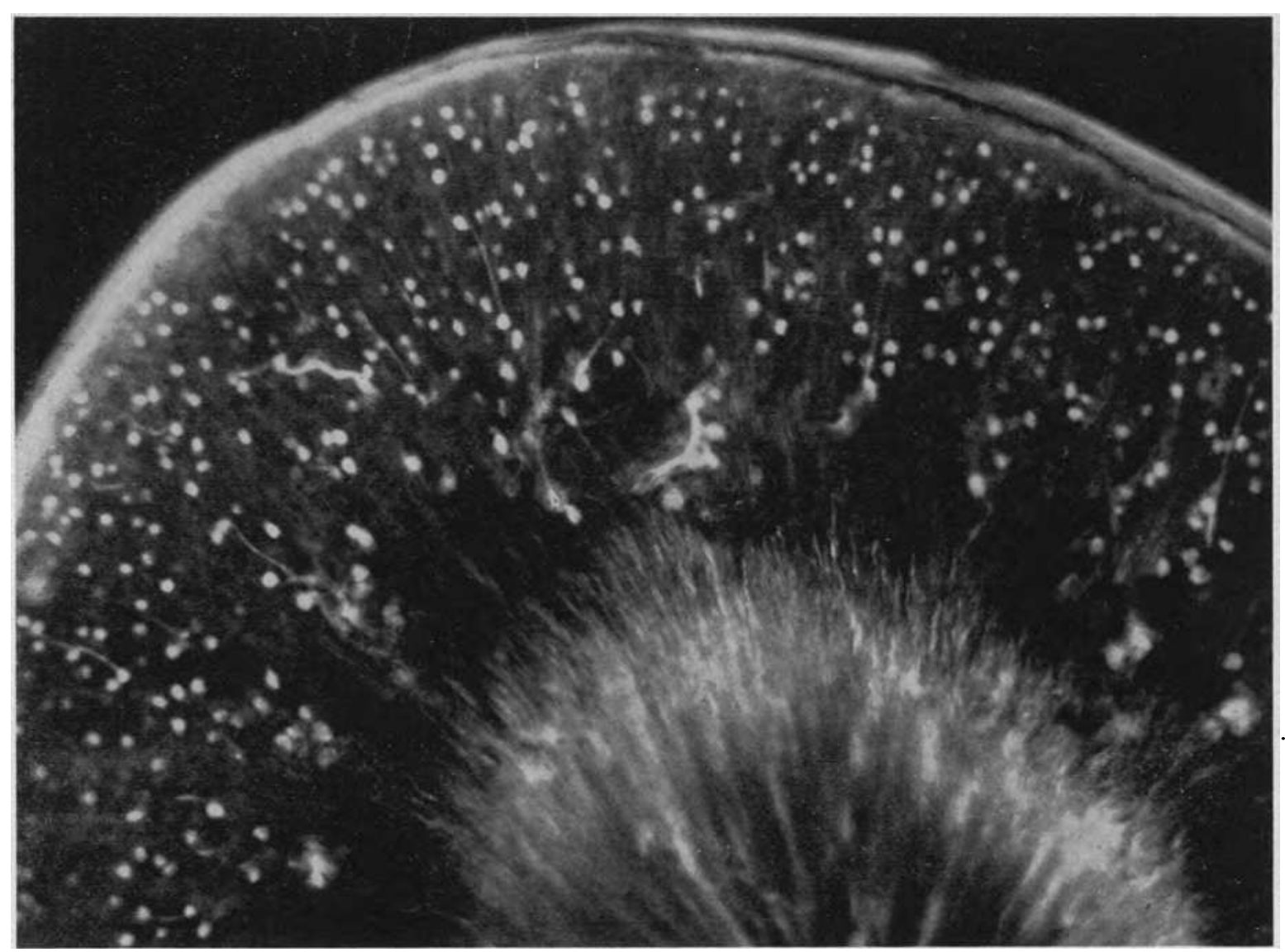

A

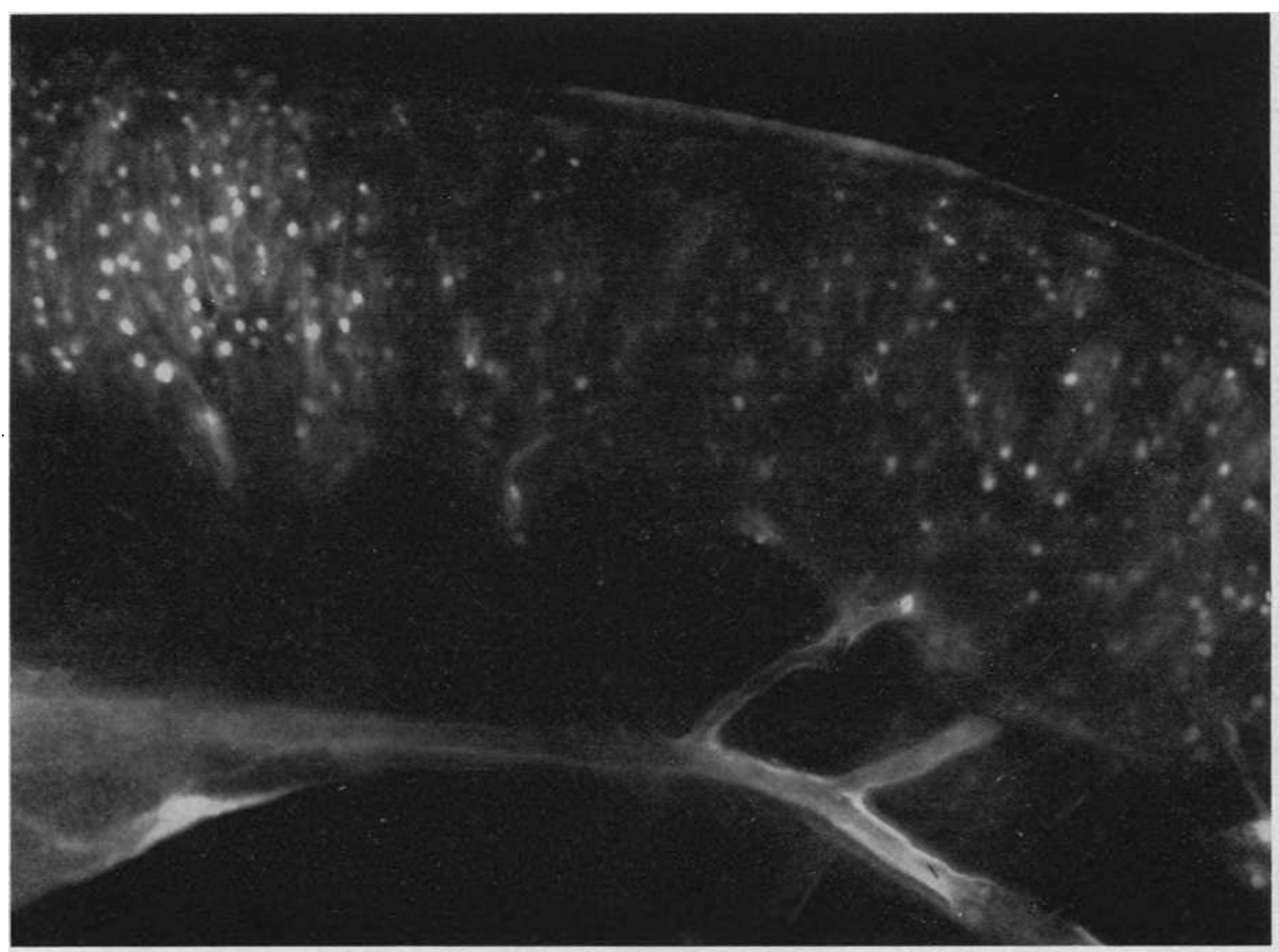




\section{Plate 38}

A. The Cortical Circulation in the Kidney of a Rabbit Which Had Been Given 24 mg. of Sublimate Intravenously 41/2 Hours Previously

There is a marked retardation of blood flow from which only occasional glomeruli received the dye-containing blood.

B. The Other Kidney of the Same Animal Which Was Allowed to Receive Blood for a Period Three Times as Long as the Former

More glomeruli and interlobular arteries have accumulated enough dye to fluoresce, but as compared to the normal pattern of 37A, the irregular patchy ischemia is still evident. Disruptive lesions in the nephrons of another animal which received the same dosage but which lived 18 hours are shown in Plate $42 \mathrm{C}$ and $\mathrm{D}$. 


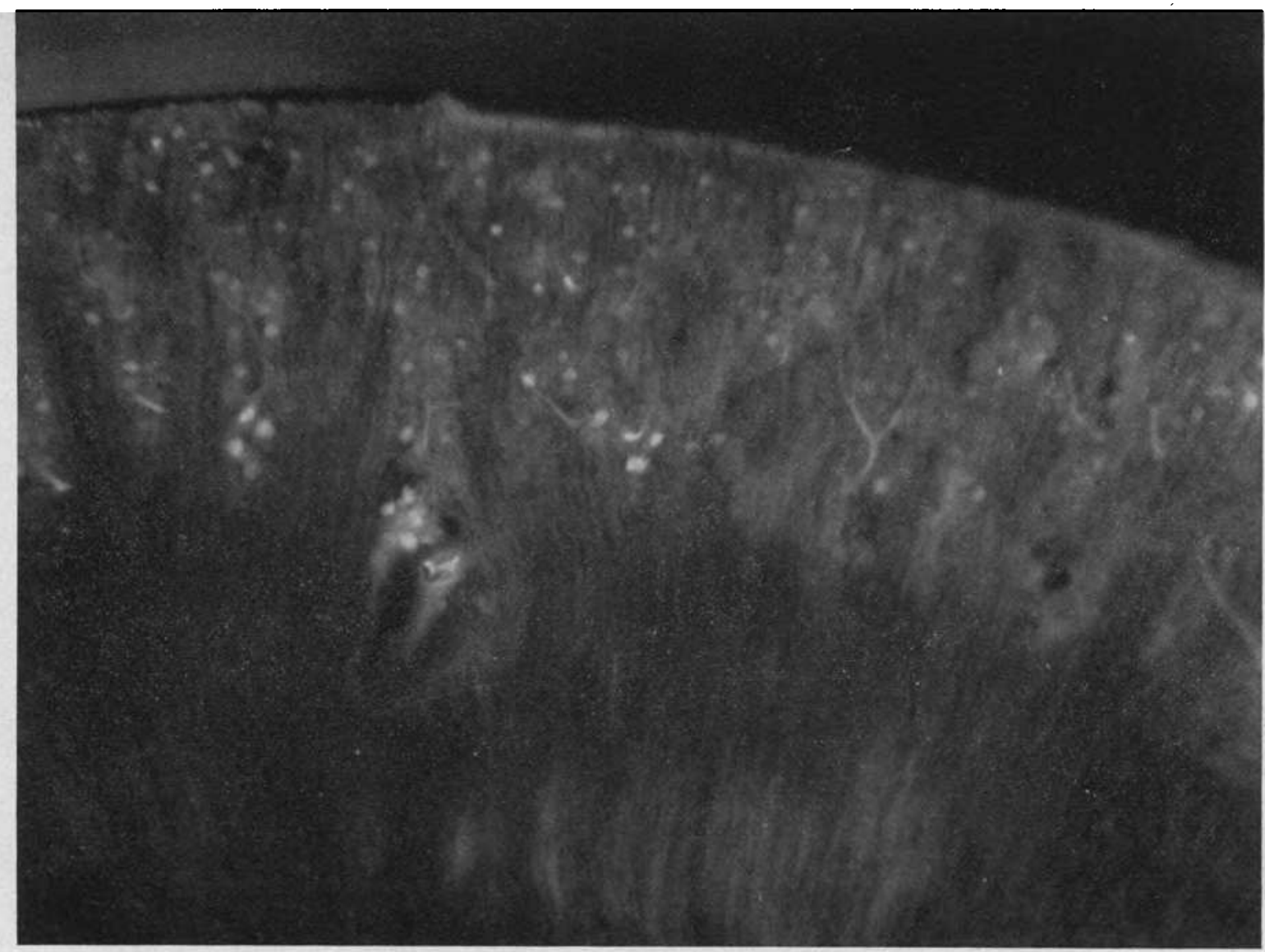

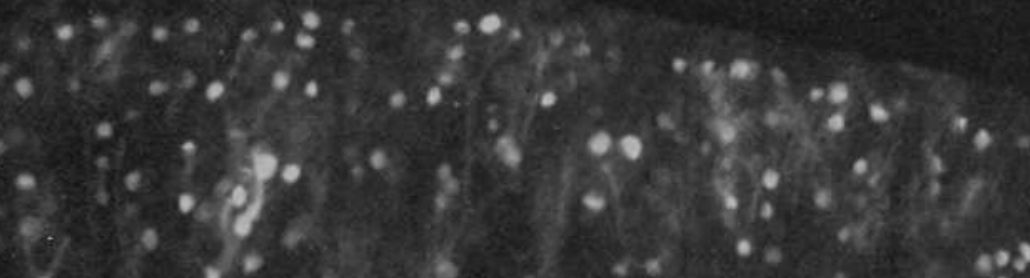

$\because \because \cdots$

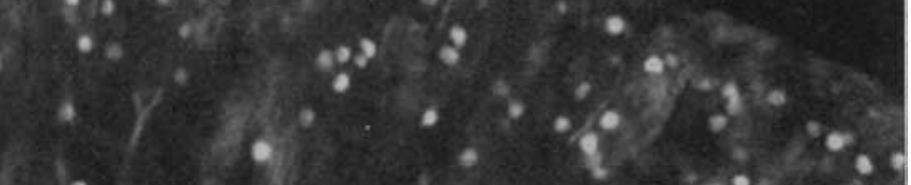

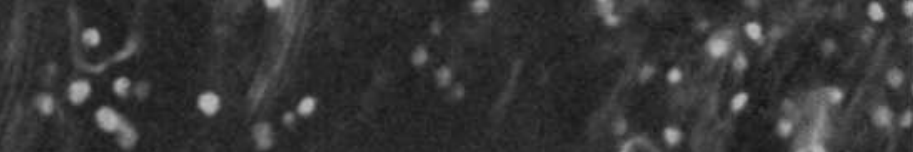

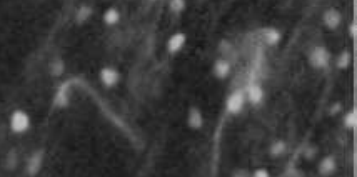

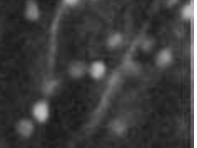

it 


\section{Plate 39}

A. The Circulatory Pattern in the Cortex of the Kinney of a Rabbit Which Had Been Given an Intravenous Injection of 40 mg. of Uranyl Nitrate 70 Hours Previously

An irregular area of ischemia with few glomeruli is seen. The exposure in this photograph and in Plate 38A was considerably longer than in the other figures hence the background of natural fluorescence of the renal tissue produced a greyish tone which is also somewhat exaggerated by the slight diffusion of the dye through vessel walls. Disruptive lesions in the nephrons of this animal are shown in Plates 42B and 43A.

B. The Cortical Circulatory Pattern in the Kidney of a Rabitt Given 30 mg. of Potassium Bichromate Intravenously 19 Hours Previously

There is a widespread but irregular ischemia of the cortex. The subcortical glomeruli, near the larger arteries, seem better irrigated. 


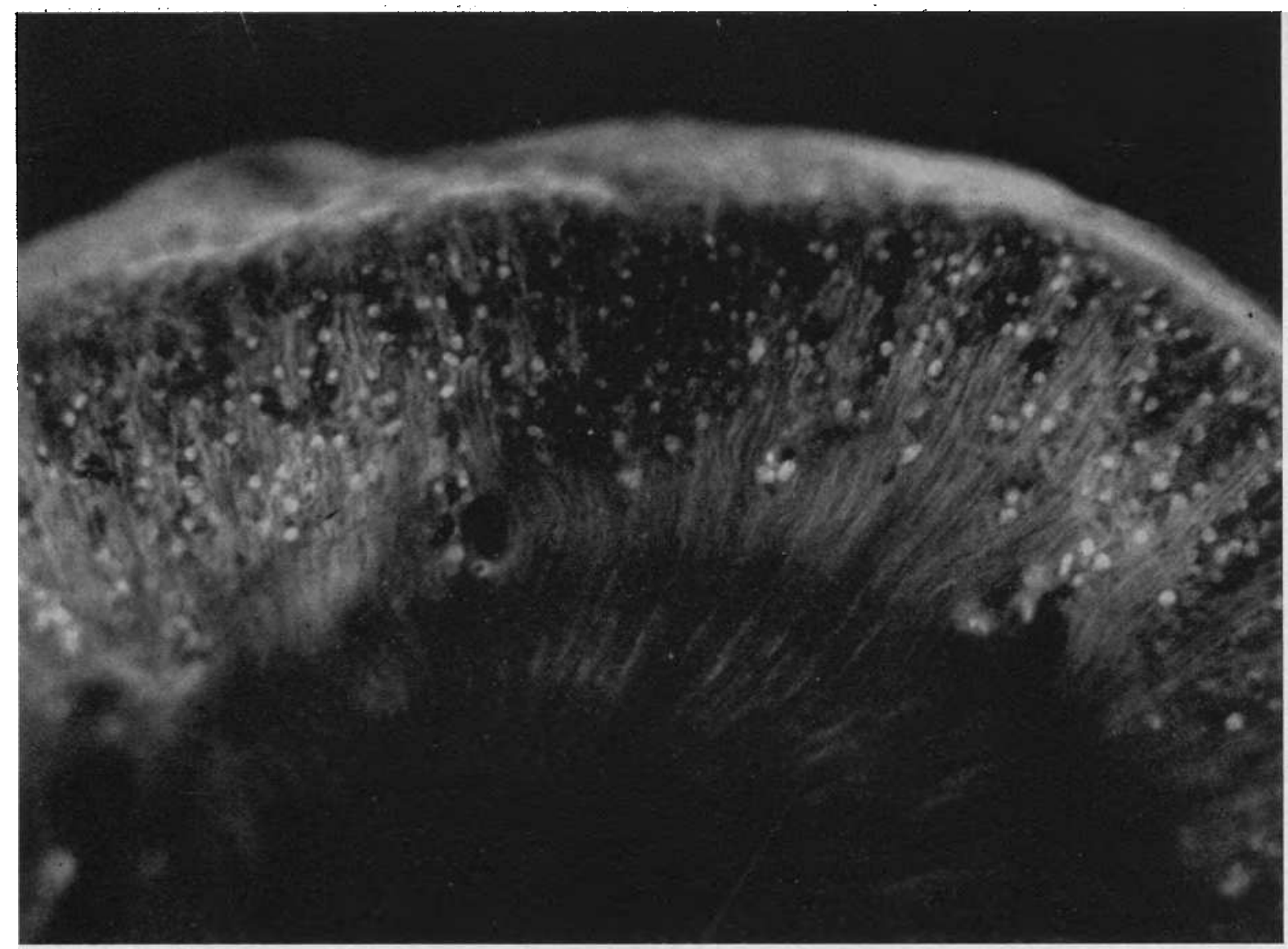

A

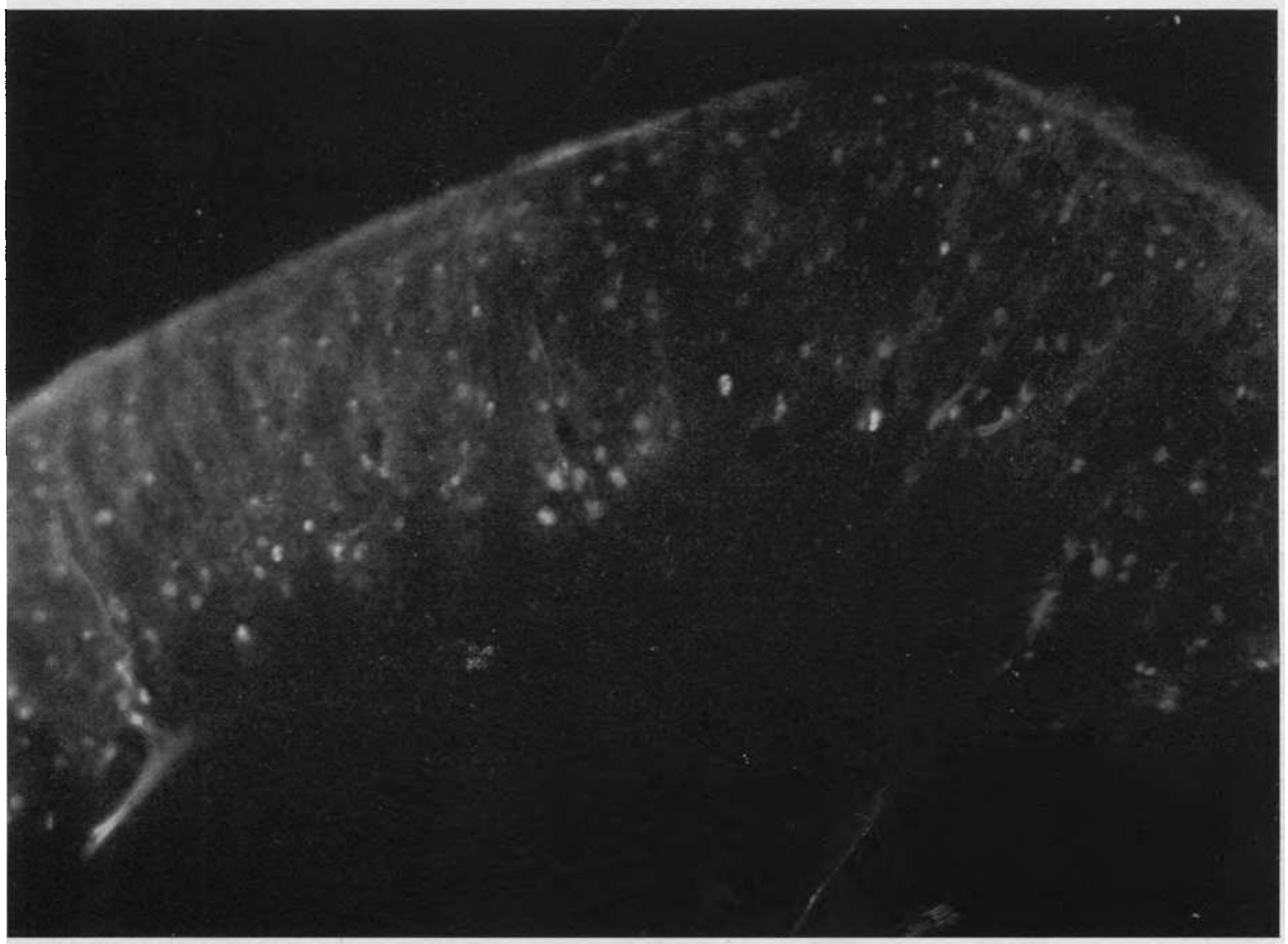




\section{Pi.ATE 40}

A. Irregulariy Patchy Cortical Ischemia is the Kidney of a Rabitt Grven 4 cc. of Carbon Tetrachloride on Two Successive Days and Injected with Dye 48 Hours Later

To the left the cortex has received a considerable amount of blood and the glomeruli and small arteries are brightly fluorescent; in the subcortical zone, particularly in the center, they can only dimly be seen. Disruptive tubular lesions from the animal are shown in Plate $43 \mathrm{D}$.

B. The Patchy Cortical Ischemia in the Kidney of a Rabbit Which Had Drunk ad lib. Di-Ethylene Glycol over a 24 Holir Period

Disruptive lesions in the nephrons of other rabbits which had drunk the same mixture over periods of five to seven days are shown in Plate 43 $\mathrm{B}$ and $\mathrm{C}$. 

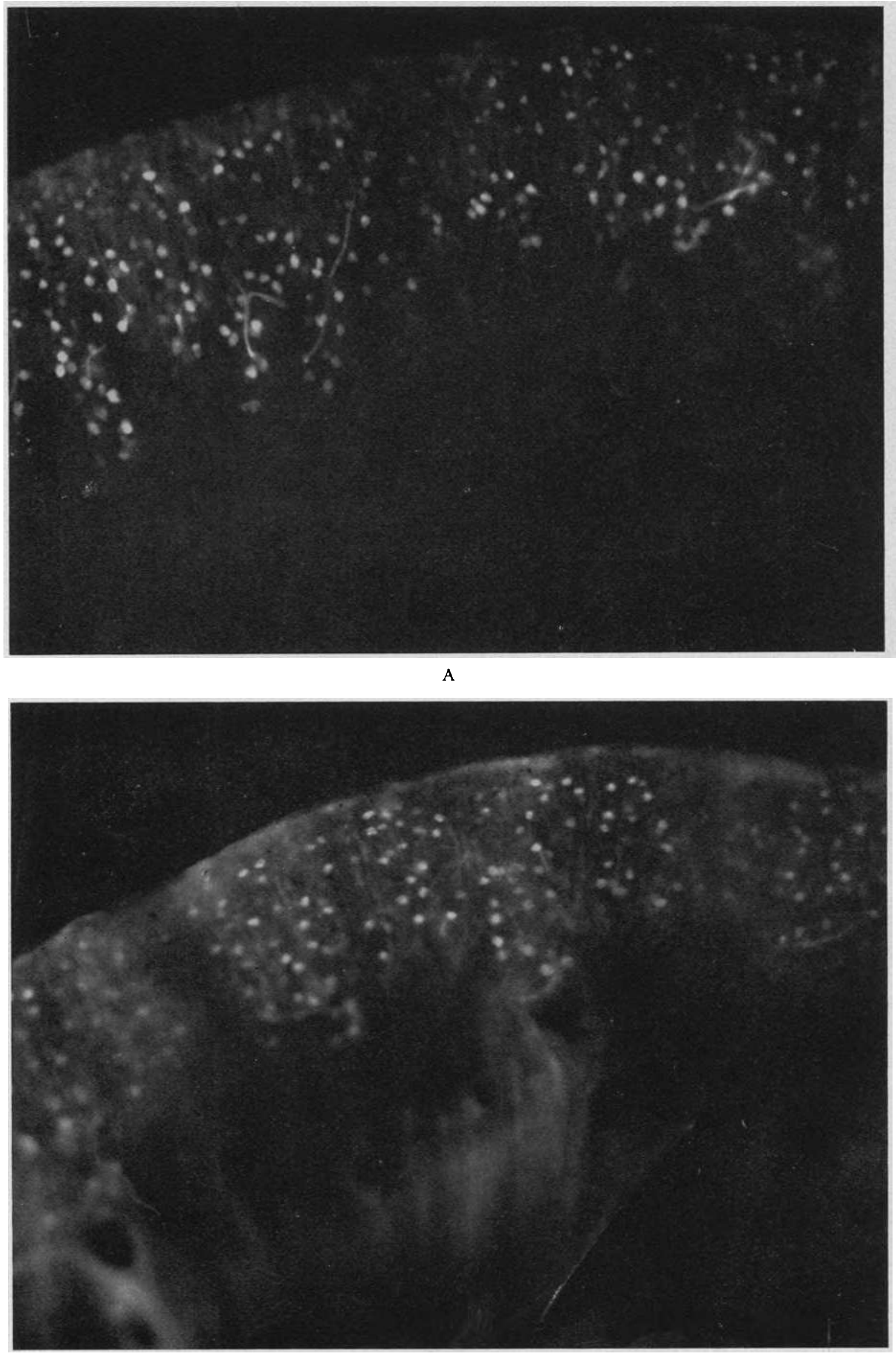
Plate 41. A Proximal Convolution and Glomerulus from a Rabit Poisoned 19 Hours Previously with 400 mg. PER kilo of Sodium Potassium Tartrate

The first few coils of the convolution are fairly normal in their nuclear and mitochondrial pattern but from the arrow distally the epithelium of the entire convolution is necrotic and reduced to cellular debris which fills the intact tube-like basement membrane. At A, however, this structure has disintegrated and typical tubular disruption has resulted. The ischemic pattern of the cortex in this animal is shown in Plate 37B. The disruptive 'lesions in the ascending limb from another animal which had received the same dosage are shown in Plate 42A. Magnification $82 \times$. 


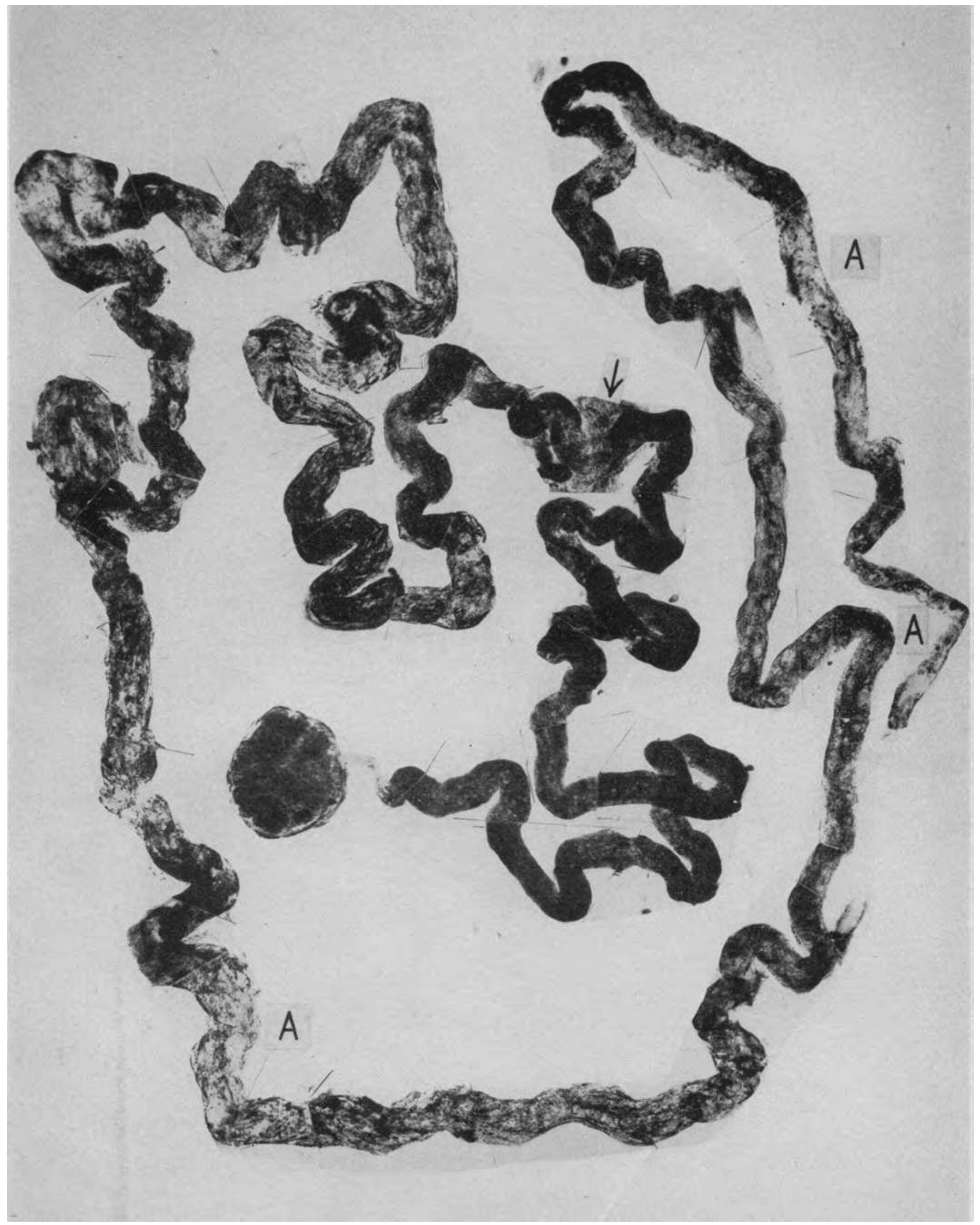




\section{Plate 42. Disruptive Tubular Lesrons Found in Animals That}

Had Received Various Toxic Agents

All of these animals showed the typical nephrotoxic necrosis in the proximal convolutions of all the nephrons along with occasional disruptive lesions in this segment. The occurrence of disruptive, but never nephrotoxic, lesions in other parts of the nephron is illustrated below. A. Sodium potassium tartrate. A greatly swollen ascending limb with disintegration of the basement membrane throughout the greater part of its length. At the arrow a more discrete disruption in the distal convolution. B. Uranyl nitrate. Basement membrane disintegration and tubular disruption at the arrows situated at the junction of the ascending limb and the distal convolution. $C$ and $D$. Corrosive sublimate. In $C$ an incipient disruption showing a localized swelling of the tubule and in $D$ another with definite basement membrane rupture. E. Di-ethylene glycol. A similar early swelling with disruption in the upper ascending limb. Magnification $80 \times$ except $B$ which is $100 \times$. 


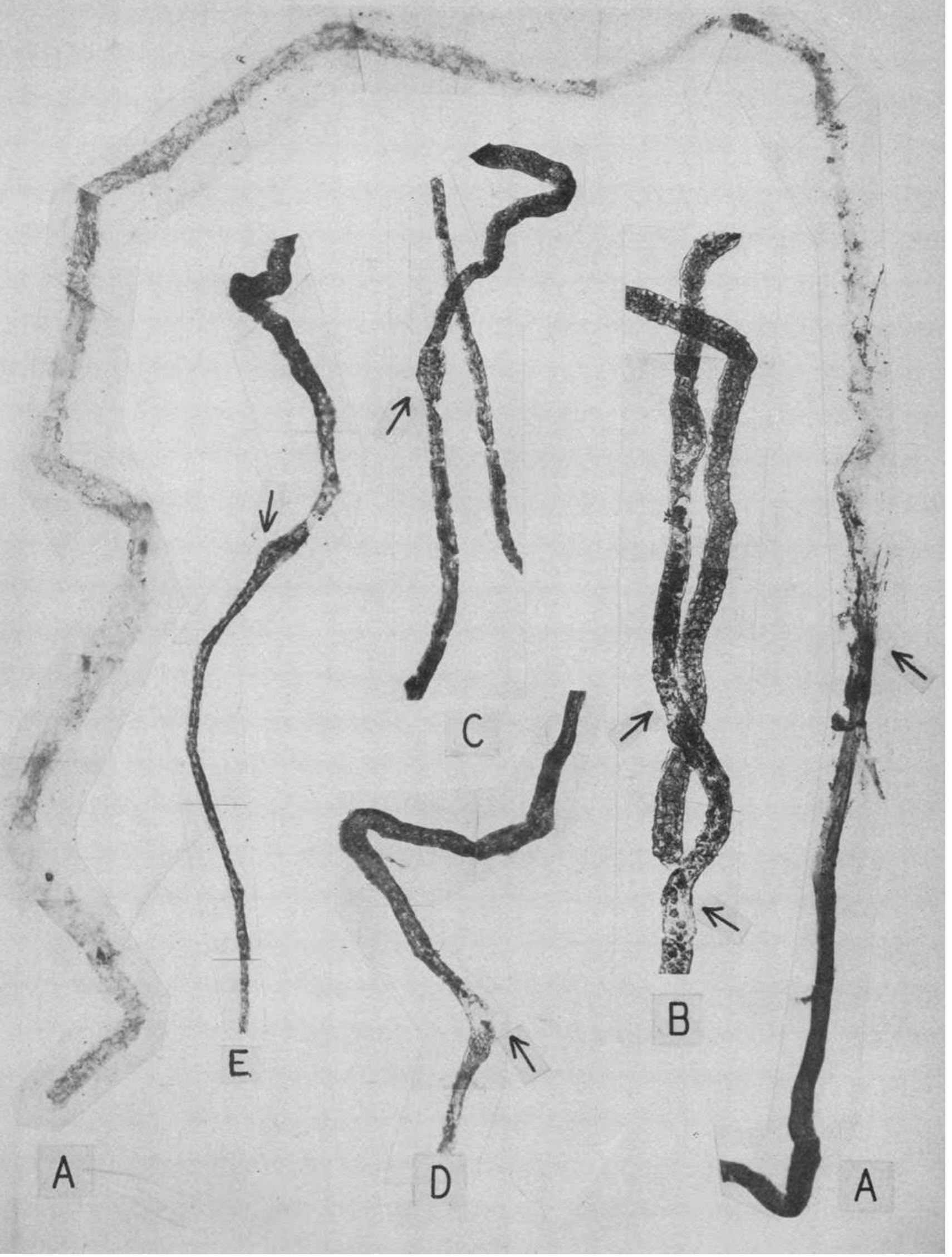




\section{Plate 43}

A. Uranyl nitrate. An ascending limb filled with cellular debris derived from the nephrotoxic damage in its preceding proximal convolution. Disruptive lesions are seen at the arrows. $B$ and $C$. Di-ethylene glycol. At $B$ an incipient lesion at the junction of ascending limb and distal convolution. In $\mathrm{C}$ a pair of ascending limbs and distal convolutions are greatly swollen and the basement membrane is almost completely disintegrated. A few necrotic cells persist in the "tubule" lumen and there is a localized disruption above where the two distal convolutions adhere. $D$. Carbon tetrachloride. Two examples of stages of the disruptive lesion in the upper ascending limbs. The dark cells mark the beginning of normally preserved distal convolutions. Magnification $80 \times$. 


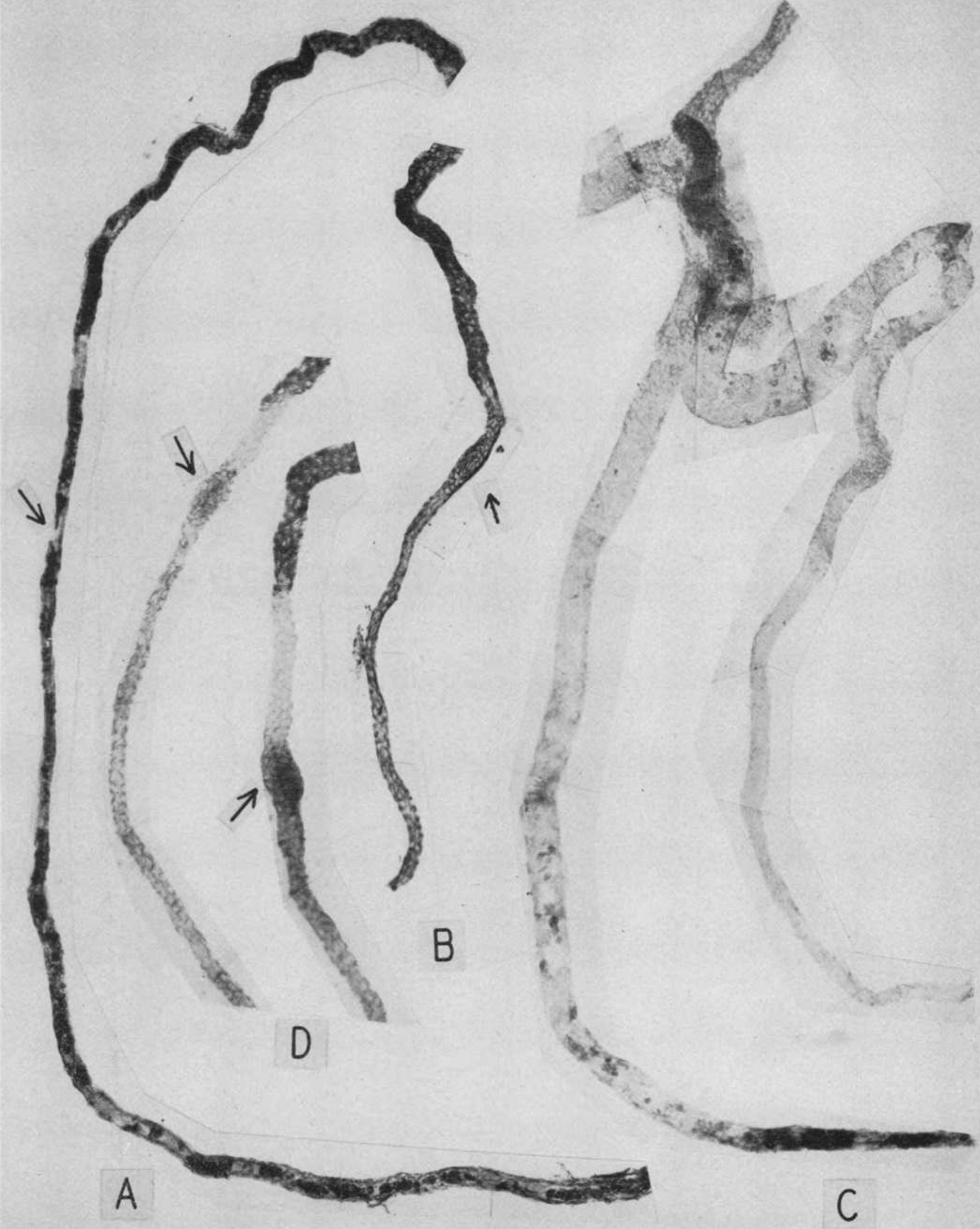


Plate 44. Proximal Convolution from a Rabbit Which Had Drcik Water ad lib. for Four Days Containing 5 Per Cent

Di-Ethylene Glycol

The localized specific effect of moderate glycol damage is seen in the extreme swelling and vacuolization of the epithelial cells of the terminal half of the proximal convolution. Magnification $82 \times$. Compare with the appearance in Figures 3 and 4 and with Plate 22 which shows more severe damage in the human kidney.

The disruptive damage in ascending limbs in this and other similarly poisoned animals is seen in Plate $42 \mathrm{E}$ in its incipient form and fully developed in Plate 43C. The pattern of cortical ischemia that accompanies glycol poisoning is shown in Plate $40 \mathrm{~B}$. 


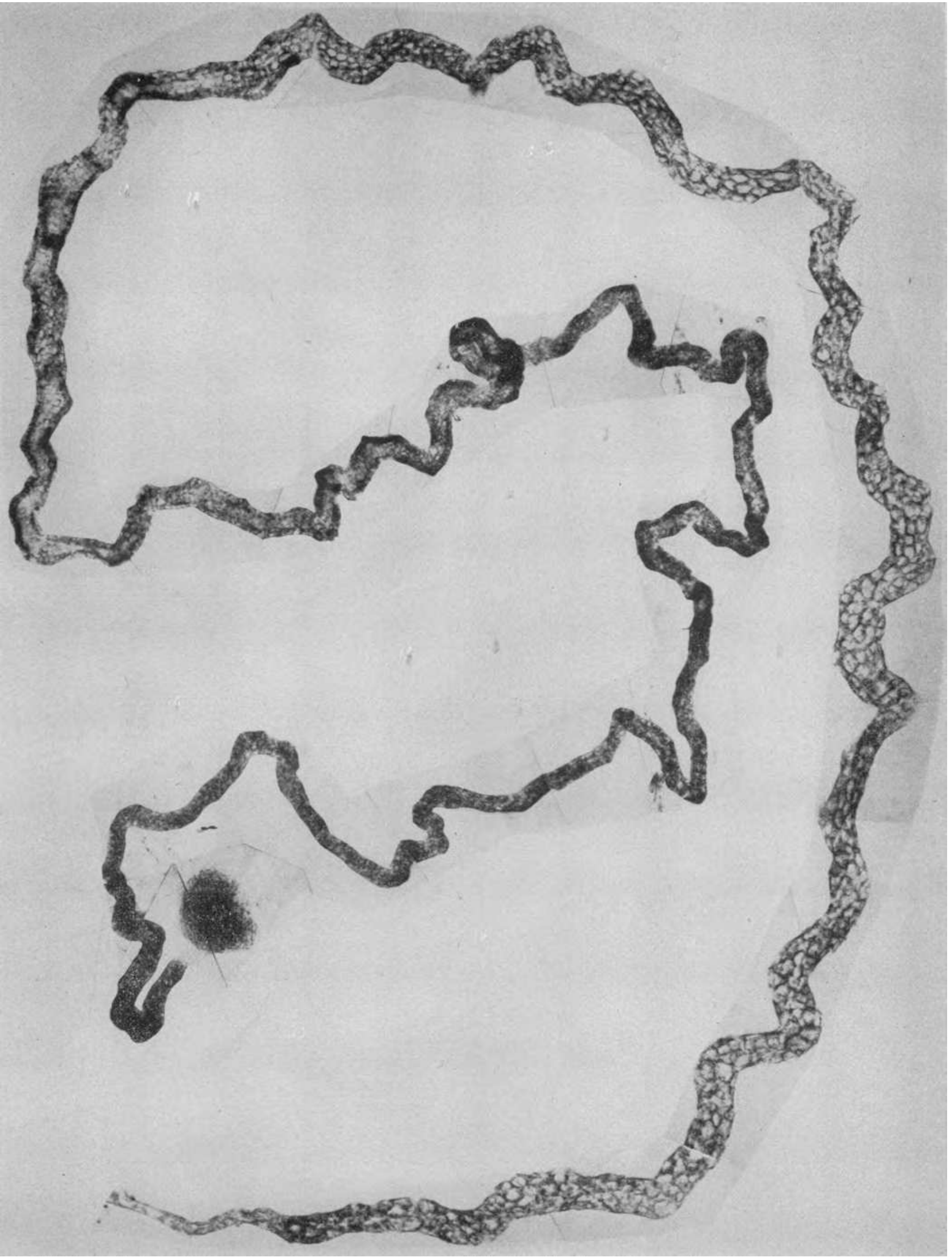

